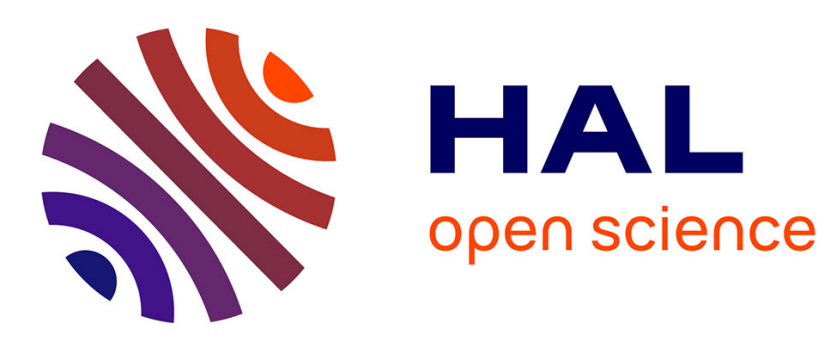

\title{
Generic polar harmonic transforms for invariant image representation
}

Thai V. Hoang, Salvatore Tabbone

\section{To cite this version:}

Thai V. Hoang, Salvatore Tabbone. Generic polar harmonic transforms for invariant image representation. Image and Vision Computing, 2014, 32 (8), pp.497 - 509. 10.1016/j.imavis.2014.04.016 . hal-01083722

\section{HAL Id: hal-01083722 \\ https://hal.inria.fr/hal-01083722}

Submitted on 17 Nov 2014

HAL is a multi-disciplinary open access archive for the deposit and dissemination of scientific research documents, whether they are published or not. The documents may come from teaching and research institutions in France or abroad, or from public or private research centers.
L'archive ouverte pluridisciplinaire HAL, est destinée au dépôt et à la diffusion de documents scientifiques de niveau recherche, publiés ou non, émanant des établissements d'enseignement et de recherche français ou étrangers, des laboratoires publics ou privés. 


\title{
Generic Polar Harmonic Transforms for Invariant Image Representation
}

\author{
Thai V. Hoang*,1 and Salvatore Tabbone ${ }^{2}$ \\ ${ }^{1}$ Inria Nancy-Grand Est, 54600 Villers-lès-Nancy, France \\ ${ }^{2}$ Université de Lorraine, LORIA, 54506 Vandouvre-lès-Nancy, France
}

\begin{abstract}
This paper introduces four classes of rotation-invariant orthogonal moments by generalizing four existing moments that use harmonic functions in their radial kernels. Members of these classes share beneficial properties for image representation and pattern recognition like orthogonality and rotation-invariance. The kernel sets of these generic harmonic function-based moments are complete in the Hilbert space of square-integrable continuous complex-valued functions. Due to their resemble definition, the computation of these kernels maintains the simplicity and numerical stability of existing harmonic function-based moments. In addition, each member of one of these classes has distinctive properties that depend on the value of a parameter, making it more suitable for some particular applications. Comparison with existing orthogonal moments defined based on Jacobi polynomials and eigenfunctions has been carried out and experimental results show the effectiveness of these classes of moments in terms of representation capability and discrimination power.
\end{abstract}

Keywords: Polar harmonic transforms; harmonic kernels; rotation invariance; orthogonal moments

\section{Introduction}

Rotation-invariant features of images are usually extracted by using moment methods [1] where an image $f$ on the unit disk $\left(x^{2}+y^{2} \leq 1\right)$ is decomposed into a set of kernels $\left\{V_{n m} \mid(n, m) \in \mathbb{Z}^{2}\right\}$ as

$$
H_{n m}=\iint_{x^{2}+y^{2} \leq 1} f(x, y) V_{n m}^{*}(x, y) \mathrm{d} x \mathrm{~d} y,
$$

where the asterisk denotes the complex conjugate. According to [2], a kernel that is "invariant in form" with respect to rotation about the origin must be defined as

$$
V_{n m}(r, \theta)=R_{n}(r) A_{m}(\theta),
$$

where $r=\sqrt{x^{2}+y^{2}}, \theta=\operatorname{atan} 2(y, x), A_{m}(\theta)=\mathrm{e}^{i m \theta}$, and $R_{n}$ could be of any form. For example, rotational moments (RM) [3] and complex moments (CM) [4] are defined by using $R_{n}(r)=r^{n}$; continuous generic Fourier descriptor (GFD) [5] employs e $\mathrm{e}^{i 2 \pi n r}$ for $R_{n}(r)$; and angular radial transform (ART) [6] uses harmonic functions as

$$
R_{n}(r)= \begin{cases}1, & n=0 \\ \cos (\pi n r), & n \neq 0 .\end{cases}
$$

\footnotetext{
${ }^{*}$ Corresponding author

E-mail address: vanthai.hoang@inria.fr, Telephone: +33 3549585 60, Fax: +33 383278319.
} 
However, the obtained kernels $V_{n m}$ of RM, CM, GFD, and ART are not orthogonal and, as a result, information redundancy exists in the moments $H_{n m}$, leading to difficulties in image reconstruction and low accuracy in pattern recognition, etc. Undoubtedly, orthogonality between kernels $V_{n m}$ comes as a natural solution to these problems. Orthogonality means

$$
\begin{aligned}
\left\langle V_{n m}, V_{n^{\prime} m^{\prime}}\right\rangle & =\iint_{x^{2}+y^{2} \leq 1} V_{n m}(x, y) V_{n^{\prime} m^{\prime}}^{*}(x, y) \mathrm{d} x \mathrm{~d} y \\
& =\int_{0}^{1} R_{n}(r) R_{n^{\prime}}^{*}(r) r \mathrm{~d} r \int_{0}^{2 \pi} A_{m}(\theta) A_{m^{\prime}}^{*}(\theta) \mathrm{d} \theta=\delta_{n n^{\prime}} \delta_{m m^{\prime}},
\end{aligned}
$$

where $\delta_{i j}=[i=j]$ is the Kronecker delta function. It can be seen from the orthogonality between the angular kernels

$$
\int_{0}^{2 \pi} A_{m}(\theta) A_{m^{\prime}}^{*}(\theta) \mathrm{d} \theta=\int_{0}^{2 \pi} \mathrm{e}^{i m \theta} \mathrm{e}^{-i m^{\prime} \theta} \mathrm{d} \theta=2 \pi \delta_{m m^{\prime}}
$$

that the remaining condition on the radial kernels is

$$
\int_{0}^{1} R_{n}(r) R_{n^{\prime}}^{*}(r) r \mathrm{~d} r=\frac{1}{2 \pi} \delta_{n n^{\prime}}
$$

This equation presents the regulating condition for the definition of a set of radial kernels $R_{n}$ in order to have orthogonality between kernels $V_{n m}$.

There exists a number of methods that have their radial kernels satisfying the condition in Eq. (1) and they can be roughly classified into three groups. The first employs Jacobi polynomials [7] in $r$ of order $n$ for $R_{n}(r)$ obtained by orthogonalizing sequences of polynomial functions or by directly using existing orthogonal polynomials. Members of this group are Zernike moments (ZM) [8], pseudo-Zernike moments (PZM) [2], orthogonal Fourier-Mellin moments (OFMM) [9], Chebyshev-Fourier moments (CHFM) [10], and pseudo Jacobi-Fourier moments (PJFM) [11] (see [12, Section 6.3], or [13, Section 3.1] for a comprehensive survey). It was demonstrated recently that the Jacobi polynomial-based radial kernels of these methods are special cases of the shifted Jacobi polynomials [14, 15]. Despite its popularity, this group of orthogonal moments however involves computation of factorial terms, resulting in high computational complexity and numerical instability, which often limit their practical usefulness.

The second group employs the eigenfunctions of the Laplacian $\nabla^{2}$ on the unit disk as $V_{n m}$, similar to the interpretation of Fourier basis as the set of eigenfunctions of $\nabla^{2}$ on a rectangular domain. These eigenfunctions are obtained by solving the Helmholtz equation, $\nabla^{2} V+\lambda^{2} V=0$, in polar coordinates to have the radial kernels defined based on the Bessel functions of the first and second kinds [16]. In addition, by imposing the condition in Eq. (1) a class of orthogonal moments is obtained [17] and different boundary conditions were used for the proposal of a number of methods with distinct definition of $\lambda$ : Fourier-Bessel modes (FBM) [18], Bessel-Fourier moments (BFM) [19], and disk-harmonic coefficients (DHC) [20]. However, the main disadvantage of these eigenfunction-based methods is the lack of an explicit definition of their radial kernels other than Bessel functions, leading to inefficiency in terms of computation complexity.

And the last group uses harmonic functions (i.e., complex exponential and trigonometric functions) for $R_{n}$ by taking advantage of their orthogonality:

$$
\begin{aligned}
\int_{0}^{1} \mathrm{e}^{i 2 \pi n r} \mathrm{e}^{-i 2 \pi n^{\prime} r} \mathrm{~d} r & =\delta_{n n^{\prime}}, \\
\int_{0}^{1} \cos (\pi n r) \cos \left(\pi n^{\prime} r\right) \mathrm{d} r & =\frac{1}{2} \delta_{n n^{\prime}},
\end{aligned}
$$




$$
\begin{aligned}
& \int_{0}^{1} \sin (\pi n r) \sin \left(\pi n^{\prime} r\right) \mathrm{d} r=\frac{1}{2} \delta_{n n^{\prime}} \\
& \int_{0}^{1} \cos (\pi n r) \sin \left(\pi n^{\prime} r\right) \mathrm{d} r=0, \quad n-n^{\prime} \text { is even, }
\end{aligned}
$$

It can be seen that the integrand in Eqs. (2)-(5) is "similar in form" with that in Eq. (1), except for the absence of the weighting term $r$ which prevents a direct application of harmonic functions as radial kernels. This obstacle was first overcome in [21] by using the multiplicative factor $\frac{1}{\sqrt{r}}$ in the radial kernels to eliminate $r$ in the definition of radial harmonic Fourier moments (RHFM) as

$$
R_{n}(r)=\frac{1}{\sqrt{r}} \begin{cases}1, & n=0 \\ \sqrt{2} \sin (\pi(n+1) r), & n>0 \quad \& n \text { is odd } \\ \sqrt{2} \cos (\pi n r), & n>0 \quad \& n \text { is even. }\end{cases}
$$

Recently, a different strategy was proposed to move $r$ into the variable of integration, $r \mathrm{~d} r=\frac{1}{2} \mathrm{~d} r^{2}$, in the definition of three different forms of polar harmonic transforms [22]: polar complex exponential transform (PCET), polar cosine transform (PCT), and polar sine transform (PST). The radial kernels of these transforms are respectively defined as

$$
\begin{aligned}
& R_{n}(r)=\mathrm{e}^{i 2 \pi n r^{2}}, \\
& R_{n}^{C}(r)= \begin{cases}1, & n=0 \\
\sqrt{2} \cos \left(\pi n r^{2}\right), & n>0\end{cases} \\
& R_{n}^{S}(r)=\sqrt{2} \sin \left(\pi n r^{2}\right), \quad n>0
\end{aligned}
$$

It is straightforward that the radial kernels in Eqs. (6)-(9) all satisfy the orthogonality condition in Eq. (1) and that their corresponding kernels are orthogonal over the unit disk. In addition, the radial kernels of RHFM in Eq. (6) are actually equivalent to $R_{n}(r)=\frac{1}{\sqrt{r}} \mathrm{e}^{i 2 \pi n r}$ in terms of image representation, similar to the equivalence between different forms of Fourier series (namely trigonometric and complex exponential functions). The resemblance between the exponential form of RHFM's radial kernels and PCET's radial kernels suggests that they are actually special cases of a generic class of radial kernels that are defined based on complex exponential functions. And each member of this class can be used to define kernels that are orthogonal over the unit disk. Similar observation also leads to generic classes of radial kernels defined based on trigonometric functions.

The main contribution of this paper is a generic view on strategies that were used to define orthogonal moments. This leads to the introduction of four classes of radial kernels that correspond to four generic sets of moments and take existing harmonic moments as special cases. This paper proves theoretically that the generic sets of kernels are complete in the Hilbert space of all square-integrable continuous complex-valued functions over the unit disk. It also shows experimentally that the proposed harmonic moments are superior to Jacobi polynomial-based moments and are comparable to eigenfunction-based moments in terms of representation capability and discrimination power. It is also interesting to note that these generic harmonic moments can be computed very quickly by exploiting the recurrence relations among complex exponentials and trigonometric functions [23]. The generalization by introducing a parameter in this paper is similar to the generalization of the $R$-transform published recently[24].

The content of this paper is a comprehensive extension of the research work presented previously in [25]. The next section will derive explicit form of generic classes of radial kernels defined based on complex exponentials and trigonometric functions. The completeness of the sets of orthogonal decomposing kernels is proven in Section 3, along with some beneficial properties obtained from the 
generalization. Section 4 is devoted to the stability of the numerical computation. Experimental results in terms of representation capability and discrimination power are given in Section 5. And conclusions are finally drawn in Section 6.

\section{Generic polar harmonic transforms}

In order to formulate the generalization, assuming that the harmonic radial kernels have the generic exponential form $R_{n s}(r)=\kappa(r) \mathrm{e}^{i 2 \pi n r^{s}}$, where $s \in \mathbb{R}$ and $\kappa$ is a real functional of $r$. Then

$$
\int_{0}^{1} R_{n s}(r) R_{n^{\prime} s}^{*}(r) r \mathrm{~d} r=\int_{0}^{1} \kappa^{2}(r) \mathrm{e}^{i 2 \pi n r^{s}} \mathrm{e}^{-i 2 \pi n^{\prime} r^{s}} r \mathrm{~d} r
$$

Since $\mathrm{d} r^{s}=s r^{s-1} \mathrm{~d} r=s r^{s-2} r \mathrm{~d} r$ then

$$
\int_{0}^{1} R_{n s}(r) R_{n^{\prime} s}^{*}(r) r \mathrm{~d} r=\int_{0}^{1} \frac{\kappa^{2}(r)}{s r^{s-2}} \mathrm{e}^{i 2 \pi n r^{s}} \mathrm{e}^{-i 2 \pi n^{\prime} r^{s}} \mathrm{~d} r^{s} .
$$

By letting $\frac{\kappa^{2}(r)}{s r^{s-2}}=$ const $=C$,

$$
\int_{0}^{1} R_{n s}(r) R_{n^{\prime} s}^{*}(r) r \mathrm{~d} r=\int_{0}^{1} C \mathrm{e}^{i 2 \pi n r^{s}} \mathrm{e}^{-i 2 \pi n^{\prime} r^{s}} \mathrm{~d} r^{s}=C \delta_{n n^{\prime}}
$$

In order to have orthonormality between kernels, it follows directly from Eq. (1) that $C=\frac{1}{2 \pi}$. Then $\kappa(r)=\sqrt{\frac{s r^{s-2}}{2 \pi}}$ and $R_{n s}$ have the following actual definition:

$$
R_{n s}(r)=\kappa(r) \mathrm{e}^{i 2 \pi n r^{s}}
$$

or

$$
V_{n m s}(r, \theta)=R_{n s}(r) A_{m}(\theta)=\kappa(r) \mathrm{e}^{i 2 \pi n r^{s}} \mathrm{e}^{i m \theta}
$$

The generic polar complex exponential transform (GPCET) is thus defined as

$$
H_{n m s}=\iint_{x^{2}+y^{2} \leq 1} f(x, y) V_{n m}^{*}(x, y) \mathrm{d} x \mathrm{~d} y=\int_{0}^{2 \pi} \int_{0}^{1} f(r, \theta) \kappa(r) \mathrm{e}^{-i\left(2 \pi n r^{s}+m \theta\right)} r \mathrm{~d} r \mathrm{~d} \theta .
$$

By considering $s$ in the above development as a parameter, it can be seen that $R_{n s}$ is a true generalization of the harmonic radial kernels of PCET [22]: $R_{n s}(r)$ in Eq. (10) becomes $R_{n}(r)$ in Eq. (7) when $s=2$, except for the constant multiplicative factor $\frac{1}{\sqrt{\pi}}$. Thus, a class of harmonic radial kernels can be obtained by changing the value of $s$. Due to the generic definition, members of this class share beneficial properties to image representation and pattern recognition. However, each member also possesses distinctive characteristics that are determined by the actual value of $s$, making it more suitable for some particular applications. Some beneficial properties of GPCET will be discussed in Section 3 and be supported by experimental evidence in Section 5. Figure 1 illustrates the phase of GPCET kernels using four different values of $s=0.5,1,2,4$ for $\left\{(n, m) \mid n, m \in[0,2],(n, m) \in \mathbb{Z}^{2}\right\}$. It can be seen that the phase of $V_{n m s}$, unlike that of the kernels defined based on polynomials, is the sum of the phase of $R_{n s}$ and $A_{m}$. The phase image of $V_{n m s}$ thus has a rotational symmetry pattern composing of repetitive slices when $n$ or $m \neq 0$. The dependence of this pattern on $n, m$, and $s$ can be described as follows:

- an increase in $n$ results in thinner and longer slices. 


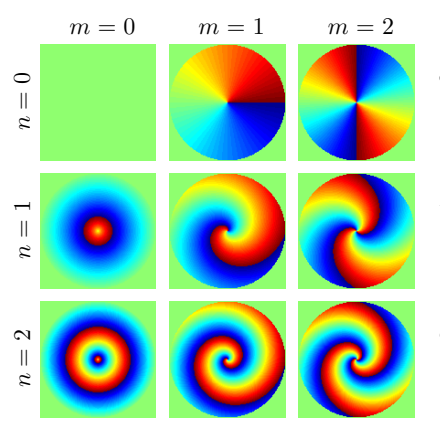

(a) $s=0.5$

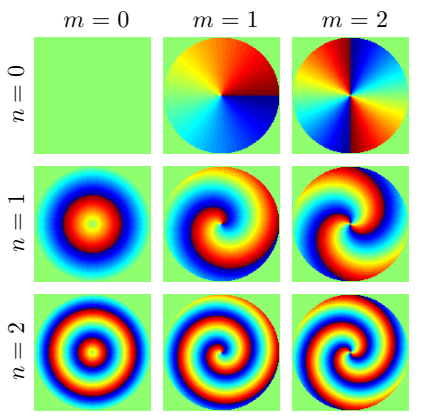

(b) $s=1$

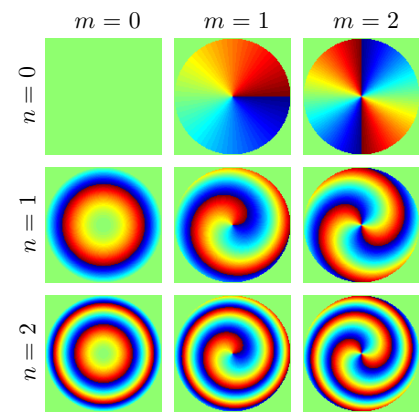

(c) $s=2$

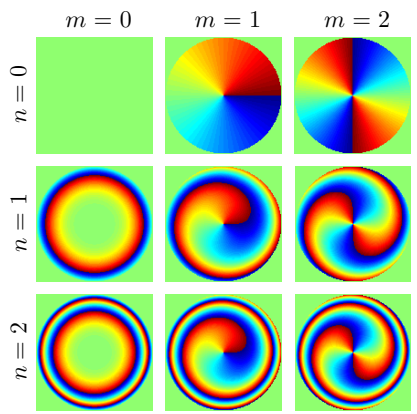

(d) $s=4$

Figure 1: 2D view of the phase of GPCET kernels $V_{n m s}$ defined in Eq. (11) using $s=0.5,1,2,4$ for $\left\{(n, m) \mid n, m \in[0,2],(n, m) \in \mathbb{Z}^{2}\right\}$. In each figure (i.e., for a specific value of $s$ ), the row and column indices indicate the values of $n=0,1,2$ (top to bottom) and $m=0,1,2$ (left to right), respectively.

- an increase in $m$ increases the number of slices.

- a change in $s$ corresponds to a change in the thickness uniformity of each slice.

In addition to the harmonic radial kernels of GPCET defined in Eq. (10), there exist three other classes of harmonic radial kernels that result from generalizing the harmonic radial kernels of RHFM $\left(R_{n s}^{H}\right)$ [21], PCT $\left(R_{n s}^{C}\right)$, and PST $\left(R_{n s}^{S}\right)$ [22]. By using similar development procedures, it is straightforward that

$$
\begin{aligned}
& R_{n s}^{H}(r)=\kappa(r) \begin{cases}1, & n=0 \\
\sqrt{2} \sin \left(\pi(n+1) r^{s}\right), & n>0 \& n \text { is odd } \\
\sqrt{2} \cos \left(\pi n r^{s}\right), & n>0 \& n \text { is even }\end{cases} \\
& R_{n s}^{C}(r)=\kappa(r) \begin{cases}1, & n=0 \\
\sqrt{2} \cos \left(\pi n r^{s}\right), & n>0\end{cases} \\
& R_{n s}^{S}(r)=\kappa(r) \sqrt{2} \sin \left(\pi n r^{s}\right), \quad n>0 .
\end{aligned}
$$

These three classes of harmonic radial kernels correspond to three classes of transforms: the generic radial harmonic Fourier moments (GRHFM), the generic polar cosine transforms (GPCT), and the generic polar sine transforms (GPST). GRHFM is in fact a variant of GPCET in terms of representation, similar to the equivalence between different forms of Fourier series. It becomes RHFM in Eq. (6) when $s=1$, except for the constant multiplicative factor $\frac{1}{\sqrt{2 \pi}}$. In addition, GPCT/GPST arise naturally from GRHFM when the function to be represented by $R_{n s}^{H}$ is considered as half of an even/odd periodic function. Again, GPCT/GPST become PCT/PST in Eqs. (8)/(9) when $s=2$, except for the constant multiplicative factor $\frac{1}{\sqrt{\pi}}$.

\section{Orthogonal sets}

At a specific value of $s,\left\langle V_{n m s}, V_{n^{\prime} m^{\prime} s}\right\rangle=\delta_{n n^{\prime}} \delta_{m m^{\prime}}$ means that

$$
\mathcal{B}_{s}=\left\{V_{n m s}(r, \theta)=R_{n s}(r) \mathrm{e}^{i m \theta} \mid n, m \in \mathbb{Z}\right\}
$$

forms a set of kernels that are orthonormal over the unit disk. Similarly, there are three other sets of orthonormal kernels at a specific value of $s$ defined as

$$
\mathcal{B}_{s}^{H}=\left\{V_{n m s}^{H}(r, \theta)=R_{n s}^{H}(r) \mathrm{e}^{i m \theta} \mid n \in \mathbb{N}, m \in \mathbb{Z}\right\},
$$




$$
\begin{aligned}
& \mathcal{B}_{s}^{C}=\left\{V_{n m s}^{C}(r, \theta)=R_{n s}^{C}(r) \mathrm{e}^{i m \theta} \mid n \in \mathbb{N}, m \in \mathbb{Z}\right\}, \\
& \mathcal{B}_{s}^{S}=\left\{V_{n m s}^{S}(r, \theta)=R_{n s}^{S}(r) \mathrm{e}^{i m \theta} \mid n \in \mathbb{Z}_{+}, m \in \mathbb{Z}\right\} .
\end{aligned}
$$

Each of the sets $\mathcal{B}_{s}, \mathcal{B}_{s}^{H}, \mathcal{B}_{s}^{C}$, and $\mathcal{B}_{s}^{S}$ can be used as the set of decomposing orthonormal kernels for GPCET, GRHFM, GPCT, and GPST, respectively. The completeness of these sets is an important issue that needs further consideration (see Section 3).

In spite of their common harmonic nature, each of GPCET, GRHFM, GPCT, and GPST captures different image information even at the same value of $s$, similar to the difference among Fourier (complex exponential and trigonometric), cosine, and sine series. This observation will have experimental evidence in Section 5. Nevertheless, in the remaining of this paper, the theoretical discussions will mainly focus on GPCET with an occasional foray into GRHFM, GPCT, and GPST only when necessary. This is to avoid unnecessary repetition, since GRHFM, GPCT, and GPST essentially have many properties that are identical to those of GPCET. In addition, if not explicitly mentioned, the parameter $s$ will have a fixed value in the remaining discussions.

\section{Properties}

This section discusses the completeness of the sets of orthogonal decomposing kernels defined in Eq. (16) along with some beneficial properties of GPCET for image representation and pattern recognition that result directly from the generalization. Other issues like relation with RM, 3D formulation, rotation invariance, rotating-angle estimation, and computational complexity could also be derived with relative ease [13, Section 3.3].

\subsection{Completeness of $\mathcal{B}_{s}$}

A set of orthogonal kernels is called complete in a Hilbert space $\mathcal{H}$ if its linear span is dense in $\mathcal{H}$. The completeness of an orthogonal set in $\mathcal{H}$ is hence related to the ability of the set in representing functions in $\mathcal{H}$. In the case of $\mathcal{B}_{s}, \mathcal{H}$ is defined as the space of all square-integrable continuous complex-valued functions over the unit disk, denoted as $\mathcal{L}^{2}\left(x^{2}+y^{2}<1\right)$. A complete $\mathcal{B}_{s}$ can be used as an orthonormal basis, meaning that every function $f \in \mathcal{H}$ can be written as an infinite linear combination of the kernels in $\mathcal{B}_{s}$ as

$$
f_{s}(x, y)=\sum_{n=-\infty}^{\infty} \sum_{m=-\infty}^{\infty} H_{n m s} V_{n m s}(x, y) .
$$

In addition, due to the Parseval's identity:

$$
\sum_{(n, m) \in \mathbb{Z}^{2}}\left|H_{n m s}\right|^{2}=\iint_{x^{2}+y^{2} \leq 1}|f(x, y)|^{2} \mathrm{~d} x \mathrm{~d} y,
$$

it can be seen that GPCET moments, $H_{n m s}$, are bounded if and only if $f$ is square-integrable. The above identity is in fact stronger than the Bessel's inequality claimed in [22, Eq. (8)], where a loose inequality is used instead of an equality. This is because [22] lacks discussion on the completeness of its proposed orthogonal sets.

In this subsection, the completeness of $\mathcal{B}_{s}$ in $\mathcal{H}$ is established by means of the interpretation of GPCET through Fourier series by rewriting Eq. (12) as

$$
H_{n m s}=\int_{0}^{2 \pi}\left[\int_{0}^{1} f(r, \theta) \kappa(r) \mathrm{e}^{-i 2 \pi n r^{s}} r \mathrm{~d} r\right] \mathrm{e}^{-i m \theta} \mathrm{d} \theta=\frac{1}{2 \pi} \int_{0}^{2 \pi}\left[\int_{0}^{1} g\left(r^{\prime}, \theta\right) \mathrm{e}^{-i 2 \pi n r^{\prime}} \mathrm{d} r^{\prime}\right] \mathrm{e}^{-i m \theta} \mathrm{d} \theta,
$$


where $r^{\prime}=r^{s}$ and

$$
g\left(r^{\prime}, \theta\right)=\sqrt{\frac{2 \pi}{s}}\left(r^{\prime}\right)^{\frac{2-s}{2 s}} f\left(\sqrt[s]{r^{\prime}}, \theta\right) .
$$

If $g$ is viewed as a 2D function defined in a Cartesian coordinate system where $r$ and $\theta$ are the horizontal and vertical axes, respectively, then the GPCET moments $H_{n m s}$ of a function $f \in \mathcal{H}$ are the 2D Fourier coefficients of $g$ formulated as above: first in the radial direction, then in the angular direction. This interpretation transforms the completeness issue of $\mathcal{B}_{s}$ in $\mathcal{H}$ into the convergence issue of 2D Fourier series, leading to the following two questions:

- The convergence of partial sums of 2D Fourier series of functions? Almost everywhere convergence of "polygonal partial sums" of $2 \mathrm{D}$ Fourier series of functions in $\mathcal{L}^{2}([0,1] \times[0,2 \pi))$ was already established in [26].

- The square-integrability of $g$ ? The necessary and sufficient conditions for the square-integrability of $g$ over the domain $[0,1] \times[0,2 \pi)$ will be established in Theorem 1 .

Theorem 1. The function $g$ defined in $E q .(21)$ is in $\mathcal{L}^{2}([0,1] \times[0,2 \pi))$ if and only if the function $f$ is in $\mathcal{L}^{2}\left(x^{2}+y^{2}<1\right)$.

Proof. From the definition of $g$ :

$$
\int_{0}^{2 \pi} \int_{0}^{1}\left|g\left(r^{\prime}, \theta\right)\right|^{2} \mathrm{~d} r^{\prime} \mathrm{d} \theta=\int_{0}^{2 \pi} \int_{0}^{1} \frac{2 \pi}{s}\left(r^{\prime}\right)^{\frac{2-s}{s}}\left|f\left(\sqrt[s]{r^{\prime}}, \theta\right)\right|^{2} \mathrm{~d} r^{\prime} \mathrm{d} \theta .
$$

By changing the variable $r=\sqrt[s]{r^{\prime}} \rightarrow r^{\prime}=r^{s}$ and $\mathrm{d} r^{\prime}=s r^{s-1} \mathrm{~d} r$, the above equation becomes

$$
\begin{aligned}
\int_{0}^{2 \pi} \int_{0}^{1}\left|g\left(r^{\prime}, \theta\right)\right|^{2} \mathrm{~d} r^{\prime} \mathrm{d} \theta & =\int_{0}^{2 \pi} \int_{0}^{1} \frac{2 \pi}{s} r^{2-s}|f(r, \theta)|^{2} s r^{s-1} \mathrm{~d} r^{s} \mathrm{~d} \theta \\
& =2 \pi \int_{0}^{2 \pi} \int_{0}^{1}|f(r, \theta)|^{2} r \mathrm{~d} r \mathrm{~d} \theta=2 \pi \iint_{x^{2}+y^{2} \leq 1}|f(x, y)|^{2} \mathrm{~d} x \mathrm{~d} y .
\end{aligned}
$$

Thus, it is straightforward that

$$
\int_{0}^{2 \pi} \int_{0}^{1}\left|g\left(r^{\prime}, \theta\right)\right|^{2} \mathrm{~d} r^{\prime} \mathrm{d} \theta<\infty \Leftrightarrow \iint_{x^{2}+y^{2} \leq 1}|f(x, y)|^{2} \mathrm{~d} x \mathrm{~d} y<\infty
$$

and the theorem is proven.

Thus, the set $\mathcal{B}_{s}=\left\{V_{n m s} \mid n, m \in \mathbb{Z}\right\}$ is complete in the Hilbert space $\mathcal{H}$ of all square-integrable continuous complex-valued functions over the unit disk $\mathcal{L}^{2}\left(x^{2}+y^{2}<1\right)$. As a result, $\mathcal{B}_{s}$ can be used as an orthonormal basis for $\mathcal{H}$ and writing $f$ as in Eq. (20) is safe (i.e., the partial sums converge to the image function). To our knowledge, there exists no such conclusion for other orthogonal sets over the unit disk where the corresponding radial kernels are defined based on polynomials or eigenfunctions.

\subsection{Zeros of $R_{n s}$}

The number of zeros of the radial kernels is an important indicator since it corresponds to the capability of moments in representing high frequency components of images. In the case of GPCET, $R_{n s}$ is defined based on complex exponential function and can be rewritten in the following form:

$$
R_{n s}(r)=\kappa(r)\left[\cos \left(2 \pi n r^{s}\right)+i \sin \left(2 \pi n r^{s}\right)\right],
$$


Table 1: The number of zeros of the $n$ th-order radial kernel of existing unit disk-based orthogonal moments.

\begin{tabular}{lclc}
\hline \hline Moments & Number of zeros & Moments & Number of zeros \\
\hline ZM & $\frac{n-m}{2}$ & BFM & $n$ \\
PZM & $n-m$ & DHC & $n$ \\
OFMM & $n$ & GPCET & $2 n$ \\
CHFM & $n$ & GRHFM & $n$ \\
PJFM & $n$ & GPCT & $n$ \\
FBM & $n$ & GPST & $n-1$ \\
\hline
\end{tabular}

and the two equations

$$
\begin{aligned}
\operatorname{real}\left(R_{n s}(r)\right) & =0, \\
\operatorname{imag}\left(R_{n s}(r)\right) & =0
\end{aligned}
$$

both have $2 n$ distinct roots in the interval $0<r<1$. For a better perception of how large this number is, Table 1 provides the number of zeros of the $n$ th-order radial kernel of existing unit disk-based orthogonal moments. It can be seen that, except for ZM, PZM, and GPCET, the $n$ th-order radial kernel of all other methods has approximately $n$ zeros. In the case of GPCET, this number is almost double whereas, for ZM and PZM, it depends on the angular order $m$. In order to have the same number of zeros $n_{0}$ as other methods, the order of the radial kernel of ZM and PZM has to be $2 n_{0}+m$ and $n_{0}+m$, respectively. These numbers are much higher than that of GPCET, which is only $\frac{n_{0}}{2}$.

In addition to the quantity, the distribution of zeros is also an important property of the radial kernels since it relates to the information suppression problem [4]. Suppression is the situation when the computed moments put emphasis on certain portions of image and neglect the rest. When the essential discriminative information is distributed uniformly over the image domain, an unfair emphasis of the extracted moments is known to have a negative impact on their discrimination power. On the contrary, when the essential discriminative information only exists in certain image portions, it is preferable to move the emphasis towards those portions. In the case of GPCET, the distribution of the zeros of $R_{n s}$ can be controlled by changing the parameter $s$. In other words, emphasis can be put on the image portions that contain this information. This is the distinctive property of GPCET that existing methods do not have.

When $s=1$, the zeros of $R_{n 1}$ are distributed uniformly, meaning a uniform emphasis over the image region. The more deviation of the value of $s$ from 1 is, the more "biased" to the inner (when $s<1$ ) or outer (when $s>1$ ) portions of the unit disk the distribution of zeros is. This in turn corresponds to the more emphasis on the inner or outer portions of image, respectively. Evidence for the observations on the quantity and distribution of zeros of $R_{n s}$ is given in Figure 2 that contains the plot of $\operatorname{real}\left(R_{n s}\right)$ and $\operatorname{imag}\left(R_{n s}\right)$ of orders $n=0 \rightarrow 4$ at $s=0.5,1,2,4$ (top row to bottom row). It can be seen that the real and imaginary parts of GPCET radial kernel of order $n$ have $2 n$ zeros in the interval $0<r<1$. Moreover, the distribution of these zeros is biased towards 0 at $s=0.5$, uniform at $s=1$, and biased towards 1 at $s=2,4$.

\section{Numerical stability}

Accuracy is another concern when moments are computed numerically. Error in the computed moments may result from the discrete approximation of continuous mathematical formulas or from the digital 

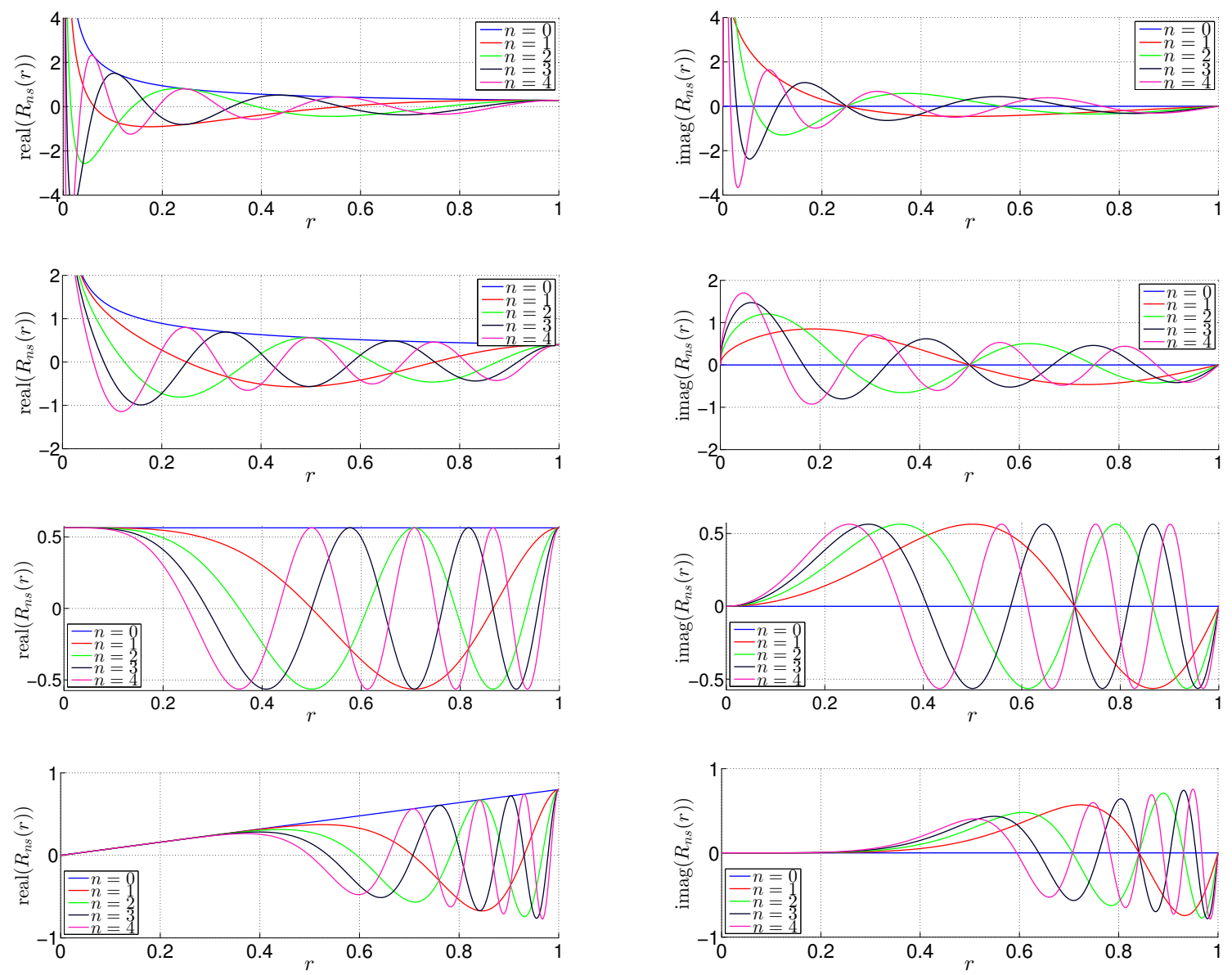

Figure 2: Real and imaginary parts of GPCET radial kernels of orders $n=0 \rightarrow 4$ at $s=0.5,1,2,4$. The real and imaginary parts of GPCET radial kernel of order $n$ have $2 n$ zeros in the interval $0<r<1$. The distribution of these zeros is uniform when $s=1$ and biased towards 0 or 1 depending on whether $s<1$ or $s>1$.

nature of computing systems, where numbers can only be represented in a certain range and to a certain precision. In addition, error also has its root in the mathematical definition of moments. These two error sources and their impacts will be discussed in the remaining of this section.

\subsection{Approximation error}

Since moments are originally defined based on a double continuous integral over the unit disk domain, the following discrete approximation of Eq. (12) will incur error in the computed moments:

$$
H_{n m s}=\sum_{[i, j] \in \mathcal{C}} f\left(x_{i}, y_{j}\right) V_{n m s}^{*}\left(x_{i}, y_{j}\right) \Delta x \Delta y,
$$

where $\mathcal{C}$ is the set of pixels whose mapped regions lie entirely inside the unit disk; $\left(x_{i}, y_{j}\right)$ are the coordinates of the center of the mapped region of pixel $[i, j]$; and $\Delta x$ and $\Delta y$ are the dimensions of each mapped region. In the above equation, there are two types of discrete approximations and they correspond to two types of approximation errors [27]: geometric error and numerical error. Geometric error occurs when the domain of integration does not exactly cover the unit disk, due to the difference between circular and rectangular domains. This type of error, however, could be "avoided" if only the pixels that lie entirely inside the unit disk are used and the image function over the remaining regions 
of the unit disk is assumed to take value 0 . Since this strategy will be used for the computation of harmonic function-based moments and comparison methods, geometric error hence does not exist in all experiments in Section 5.

Numerical error arises when $V_{n m s}^{*}\left(x_{i}, y_{j}\right) \Delta x \Delta y$ in Eq. (22), which represents the value of the kernel $V_{n m s}$ over the pixel $[i, j]$, is computed by a numerical integration technique. Since the numerically computed value of $V_{n m s}^{*}\left(x_{i}, y_{j}\right) \Delta x \Delta y$ is just an approximation to its analytical value, this type of error cannot be avoided in any way if one chooses to compute moments by numerical approximation. The magnitude of this type of error, however, could be reduced if only a highly accurate numerical integration technique is employed (e.g., "pseudo" sub-sampling or cubature). It can be seen from the factor $\Delta x \Delta y$ that the effect of numerical error depends on image size: a smaller-sized image will have a more severe effect, and vice versa. The effect of numerical error on harmonic function-based moments and comparison methods will be demonstrated experimentally by means of reconstruction error in Section 5 .

\subsection{Representation error}

In computing systems nowadays, a real number is in general approximately represented in floating-point format in order to allow reasonable storage requirement and relatively quick calculations. The typical number that can be represented exactly is of the form:

$$
\text { Significand } \times \text { base } \text { exponent }^{\text {ex }}
$$

where significand denotes a signed digit string of a given length in a given base and exponent is a signed integer which modifies the magnitude of the number. As computing systems are binary in nature, floating-point numbers are normalized for representation as $\pm(1+f) \times 2^{e}$, where $f$ is the fraction or mantissa $(0 \leq f<1)$ and $e$ is the exponent. In 32-bit computers that use the IEEE 754 standard, double precision floating numbers occupy two storage locations, or 64 bits, to store the value of $f, e$, and the sign: 52 bits for $f, 11$ bits for $e+1023$, and 1 bit for the sign. A double number $v$ thus can only be represented with the relative accuracy of one-half the machine epsilon, or $\frac{1}{2} \times e p s=\frac{1}{2} \times 2^{-52} \simeq 1.1102 \times 10^{-16}$. This means that, when represented in the ordinary decimal numeral system, only the first 15 leftmost digits of $v$ are significant. Because of the limited range of $e$, the absolute values of double numbers are additionally limited in the range $2^{-1022} \div(2-e p s) 2^{1023}$, or approximately $2.2251 \times 10^{-308} \div 1.7977 \times 10^{308}$. This finite set of double numbers with finite precision leads to the phenomena of underflow, overflow, and roundoff in computing systems. Due to their nature, it is known in the literature that Jacobi polynomial-based methods suffer from all three types of errors [28] as pointed out below.

Underflow error occurs when an absolute value of a computed quantity (except zero) is under the range of its data type. Jacobi polynomial-based methods have this type of error due to the use of powers of $r$ in their definition. At a radial coordinate $r$ that is close to zero, let's say $r=0.001$, $r^{102}=1.0000 \times 10^{-306}$ and $r^{103}=1.0000 \times 10^{-309}$. Thus, any computation that involves $r$ to the power greater than 102 will cause underflow error. It is obvious that this type of error depends on the size of images: a larger-sized image starts to have this error at a lower order. As an example, for an input image of size $1024 \times 1024$ pixels, the smallest value of $r$ in the computation is $\frac{1}{1024}=2^{-10}=9.7656 \times 10^{-4}$, underflow error will start to occur at $n=103$ onwards for all Jacobi polynomial-based methods.

Overflow error occurs when a computed quantity has a value above the range of its data type. Jacobi polynomial-based methods has this type of error due to the use of factorials in their definition. Since $170 !=7.2574 \times 10^{306}$ and $171 !=1.2410 \times 10^{309}$, any computation that involves factorial of a number greater than 170 will cause overflow error. From the definition of Jacobi polynomial-based radial kernels, it is straightforward that ZM, PZM, OFMM, CHFM, and PJFM start to have this type of error at $n=171,85,85,171$, and 84 onwards, respectively. 
Table 2: The radial orders of Jacobi polynomial-based methods from which underflow, overflow, and roundoff errors start to occur for an input image of size $1024 \times 1024$ pixels.

\begin{tabular}{lccccc}
\hline \hline Error type & ZM & PZM & OFMM & CHFM & PJFM \\
\hline Underflow & 103 & 103 & 103 & 103 & 103 \\
Overflow & 171 & 85 & 85 & 171 & 84 \\
Roundoff & 46 & 23 & 23 & 79 & 21 \\
\hline
\end{tabular}

Roundoff error is the difference between an approximation of a number used in computation and its exact (i.e., correct) value. Because of the finite precision in computing systems, this type of error occurs in almost all numerical computation steps. However, Jacobi polynomial-based methods face the problem of large polynomial's coefficients in their radial kernels. These coefficients are sometimes larger than $2^{52}$ and thus, for the commonly 15-digit precision, computing radial kernels produces error of the order of unity or larger. It is not difficult to determine the order where each Jacobi polynomial-based method starts to have this type of error. They are $n=46,23,23,79$, and 21 for ZM, PZM, OFMM, CHFM, and PJFM, respectively.

The starting orders for each type of error for all Jacobi polynomial-based methods are collected and given in Table 2. Due to their distinct definition, it can be seen that different methods have different orders for overflow and roundoff errors. For underflow error, Jacobi polynomial-based methods have the same order because their radial kernels of the same order have the same polynomial order. Among these three types of representation errors, roundoff error occurs at the smallest order for each Jacobi polynomial-based method. Thus, roundoff error is the main concern in moment computation.

From the above definition of three types of representation errors, it can be seen that eigenfunctionbased and harmonic function-based methods do not suffer from the underflow and overflow errors. They do have roundoff error because of the nature of numerical computing systems. However, the effect of roundoff error on them is not as severe as on Jacobi polynomial-based methods because their definition does not use large-valued coefficients. In contrast, this effect causes serious problems in Jacobi polynomial-based methods, as will be shown experimentally in the next section. Nevertheless, any of the aforementioned error types is undesirable since it alters the computed values of moments, compromises the orthogonality of moments/kernels, and finally corrupts the overall performance of applications.

\subsection{Singularity}

In theory, GPCET could be defined as in Eq. (12) for every $s \in \mathbb{R}$. However, because the multiplicative term is defined as $\kappa(r)=\sqrt{\frac{s r^{s-2}}{2 \pi}}$, it should be aware that

$$
\lim _{s<2, r \rightarrow 0} \sqrt{\frac{s r^{s-2}}{2 \pi}}=+\infty
$$

Evidence for this behavior can be seen in Figure 2 for the cases $s=0.5$ and $s=1$ where the magnitude of the real and imaginary parts of GPCET radial kernels go to infinity as $r \rightarrow 0$. These phenomena also exist in the other harmonic function-based methods and in CHFM. However, this property does not result in "big" problems because the actual computation is carried out by using Eq. (22), instead of Eq. (12). As long as the center $\left(x_{i}, y_{j}\right)$ of the pixel's mapped region does not coincide $(0,0)$, the computed moments are bounded and hence harmonic function-based methods with $s<2$ and CHFM can still be used for image representation and in pattern recognition problems. The practical usefulness of these methods will be demonstrated by experiments in the following section. 


\section{A N}

(a) Vector character images

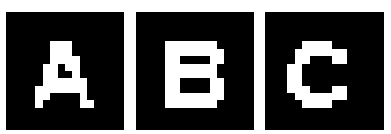

(b) $16 \times 16$

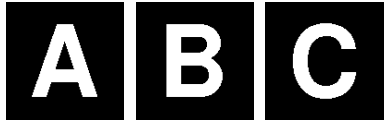

(e) $128 \times 128$

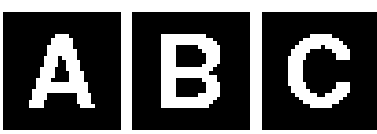

(c) $32 \times 32$

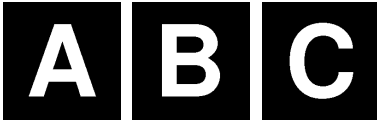

(f) $256 \times 256$

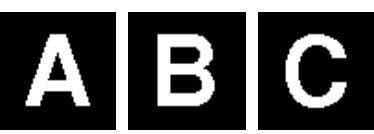

(d) $64 \times 64$

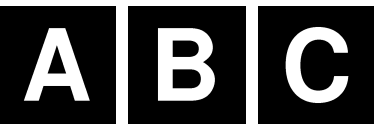

(g) $512 \times 512$

Figure 3: (a) The vector character images used to generate six character datasets used in the reconstruction experiments by sampling these images to have the sizes of $16 \times 16,32 \times 32,64 \times 64,128 \times 128$, $256 \times 256$, and $512 \times 512$ pixels, corresponding to the six datasets. (b)-(g) Some sampled images from the six datasets.

\section{Experimental results}

The effectiveness of the proposed harmonic function-based moments will be demonstrated in comparison with existing moments of the same nature, i.e. unit disk-based orthogonal moments, through two types of experiments: image representation and pattern recognition. The first deals with the capability of harmonic function-based moments in representing pattern images and is done via image reconstruction. The second is on the applicability of harmonic function-based moments in rotation-invariant pattern recognition problems at different levels of noise.

\subsection{Image reconstruction and numerical stability}

In the following experiments, a set of six character datasets has been generated by sampling 26 vector images of Latin characters in Arial bold font (shown in Figure 3a) to have the sizes of $16 \times 16,32 \times 32$, $64 \times 64,128 \times 128,256 \times 256$, and $512 \times 512$ pixels. The purpose of using datasets of images of different sizes generated from the same source is to investigate the influence of numerical error discussed in Section 4 on the computed values of moments of comparison methods. The representation error, which exists in Jacobi polynomial-based methods, will become apparent when moments of high-enough radial orders are involved. Some samples of reconstructed images from the character image "E" of size $64 \times 64$ pixels by GPCET are given in Figure 4. The corresponding images of harmonic function-based (GPCET, GPCT, GPST), Jacobi polynomial-based (ZM, PZM, OFMM, CHFM, PJFM), and eigenfunction-based (FBM, BFM, DHC) methods are given in Figures 1 and 2 in the Supplemental material. In these figures, at each value of $K$, all moment orders $(n, m)$ that satisfy the conditions in Table 3 are used for reconstruction. These conditions are selected so that the moments that capture the lowest frequency information are used first in the reconstruction process.

Generally, as more moments are involved, the reconstructed images get closer to the original ones. However, in the case of PZM, OFMM, and PJFM, reconstructed images deteriorate quickly at $K=23$, 23, and 21 onwards, respectively. Similar phenomena also exist in other Jacobi polynomial-based methods but at a higher value of $K$ (46 for ZM and 79 for CHFM). Harmonic function-based methods 
Table 3: The constraints on moment order $n$ and repetition $m$ of comparison methods with regard to $K$.

\begin{tabular}{cc}
\hline \hline Moments & Order range \\
\hline ZM & $|m| \leq n \leq K, n-|m|=$ even \\
PZM & $|m| \leq n \leq K$ \\
OFMM/CHFM/PJFM & $0 \leq|m|, n \leq K$ \\
FBM/BFM/DHC & $0 \leq|m|, n \leq K$ \\
GPCET & $|m|,|n| \leq K$ \\
GRHFM & $|m| \leq K, 0 \leq n \leq 2 K$ \\
GPCT & $0 \leq|m|, n \leq K$ \\
GPST & $|m| \leq K, 1 \leq n \leq K$ \\
\hline
\end{tabular}

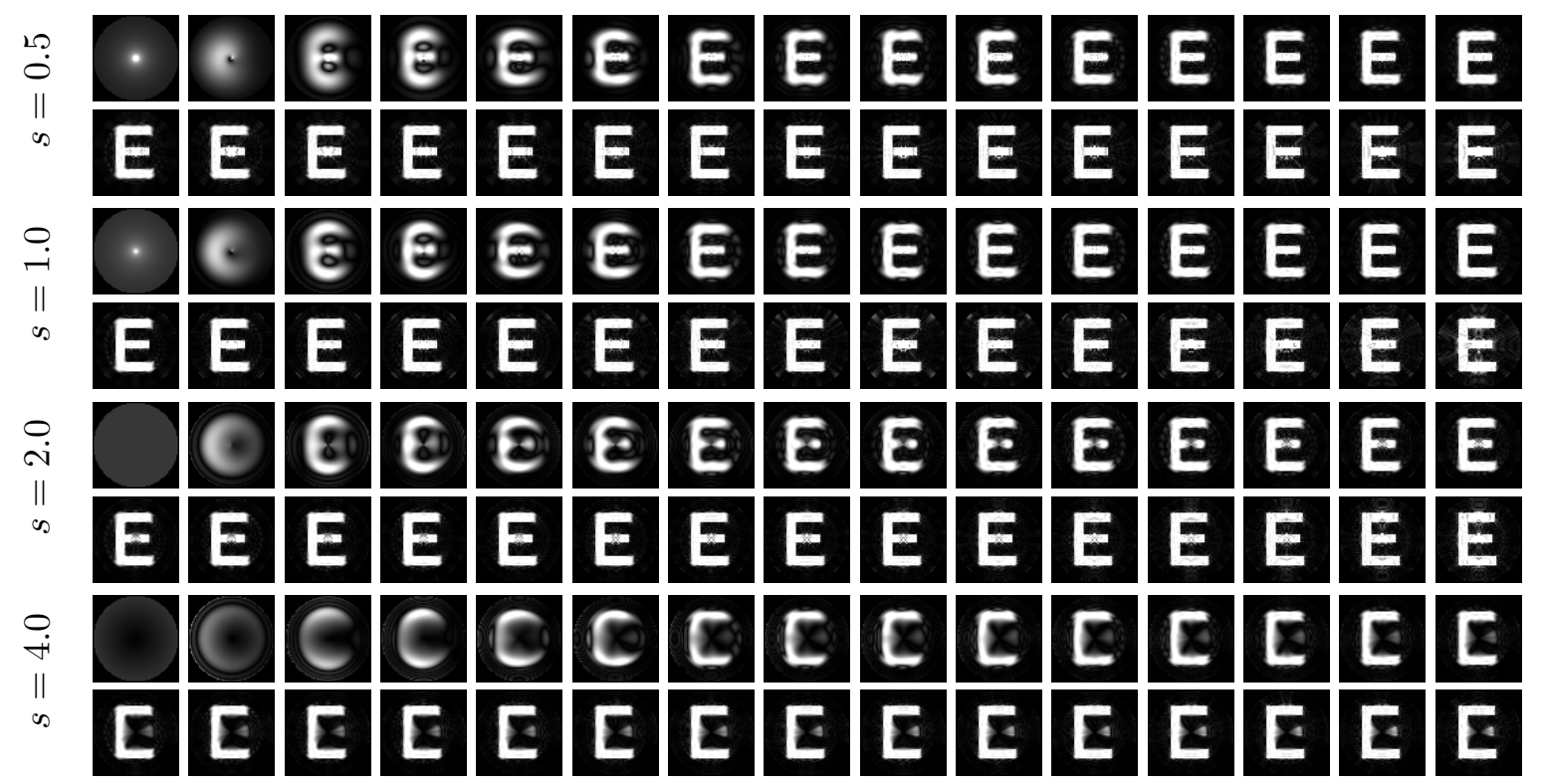

Figure 4: Some samples of reconstructed images from the character image "E" of size $64 \times 64$ pixels by GPCET at $s=0.5,1,2,4$ for $K=0,1, \ldots, 29$ (from left to right, top to bottom).

have difficulty in restoring the inner portion of the images when $s=2,4$ with more difficulty at $s=4$. On the contrary, they have difficulty with the images' outer portion when $s=0.5$. This is the experimental evidence for the information suppression problem mentioned in Subsection 3.2. Among harmonic function-based methods and at a specific value of $s$, GPCET has better reconstructed images when $K$ is small. At high values of $K$, images reconstructed by GPCT/GPST are closest/farthest to the original images at the corresponding values of $K$. This means that GPCT/GPST require the least/largest numbers of moments in order to reconstruct images of similar quality. These superiority/inferiority of GPCT/GPST can be easily observed at boundary regions where $r \simeq 0$ and $r \simeq 1$. In addition, harmonic function-based and eigenfunction-based methods capture the image information, especially the edges, better than Jacobi polynomial-based methods. It thus can be concluded here that the more deviation the value of $s$ from 1 is, the more difficulty harmonic function-based methods will have to reconstruct the inner (when $s>1$ ) or outer (when $s<1$ ) portions of images. Conversely, harmonic function-based methods can reconstruct quickly the inner or outer portions of images when $s<1$ or $s>1$, respectively. In other words, the parameter $s$ could be used to control the representation capability of harmonic function-based methods: more emphasis could be placed on certain regions of interest. 
Table 4: The cardinality $|\mathcal{S}(K)|$ of the set $\mathcal{S}(K)=\{(n, m) \mid n, m \in \mathbb{Z}\}$ of comparison methods at a specific value of $K$.

\begin{tabular}{cc}
\hline \hline Moments & $|\mathcal{S}(K)|$ \\
\hline ZM & $\frac{(K+1)(K+2)}{2}$ \\
PZM & $(K+1)^{2}$ \\
OFMM/CHFM/PJFM & $(K+1)(2 K+1)$ \\
FBM/BFM/DHC & $(K+1)(2 K+1)$ \\
GPCET & $(2 K+1)^{2}$ \\
GRHFM & $(2 K+1)^{2}$ \\
GPCT & $(K+1)(2 K+1)$ \\
GPST & $K(2 K+1)$ \\
\hline
\end{tabular}

The gauge of reconstruction capability is measured by how well the reconstructed image is similar to the ground-truth one. For this purpose, the reconstruction error between an image and its reconstructed version is considered to be a good measure. In order to compute it, a finite set of moments is first calculated and then images are reconstructed from them in order to compute the errors. Since this process involves the computation of moment kernels both in the decomposition and then reconstruction steps, this measure can additionally be used for the investigation of the numerical stability of comparison methods. Let $\mathcal{S}(K)$ be the set containing all $(n, m)$ that satisfy the conditions stated in Table 3 at a specific value of $K$. Table 4 provides the cardinality $|\mathcal{S}(K)|$ of $\mathcal{S}(K)$ of comparison methods. For an image function $f$ defined over the region $\left\{(x, y) \in \mathbb{R}^{2} \mid x^{2}+y^{2} \leq 1\right\}$, its reconstructed version by using all moments of orders $(n, m) \in \mathcal{S}(K)$ is defined as

$$
\hat{f}_{s}(x, y)=\sum_{(n, m) \in \mathcal{S}(k)} H_{n m s} V_{n m s}(x, y)
$$

The reconstruction error, normalized by the total image energy, is then defined as

$$
\operatorname{MSRE}(K)=\frac{E\left\{\iint_{x^{2}+y^{2} \leq 1}\left[f(x, y)-\hat{f}_{s}(x, y)\right]^{2} \mathrm{~d} x \mathrm{~d} y\right\}}{E\left\{\iint_{x^{2}+y^{2} \leq 1} f^{2}(x, y) \mathrm{d} x \mathrm{~d} y\right\}},
$$

where $E\{\cdot\}$ is the expectation in ensemble averaging over the image set. In the literature, $\operatorname{MSRE}(K)$ is called the mean-square reconstruction error [3]. It is straightforward to show theoretically that $0 \leq \operatorname{MSRE}(K) \leq 1$. The lower (upper) bounds of $\operatorname{MSRE}(K)$ are reached when $|\mathcal{S}(K)|$ reaches its limits, or $|\mathcal{S}(K)|=0(\infty)$. However, because of the numerical/representation errors and the unreachable theoretical point $|\mathcal{S}(K)|=\infty$, the statement $0 \leq \operatorname{MSRE}(K) \leq 1$ does not hold. Instead, it can only be asserted that $\operatorname{MSRE}(K)>0$. In this experiment, a smaller value of $\operatorname{MSRE}(K)$ means the reconstructed image $\hat{f}_{s}$ is more similar to $f$ or, in other words, a better reconstruction. In addition, by simple observation, $\operatorname{MSRE}(K)$ should have a smaller value when more moments are used in the reconstruction process, regardless of their orders.

The MSRE $(K)$ curves of GPCET on the six character datasets at different values of $s=0.1: 0.1: 6.0$ in MATLAB's notation are given in Figure 5. The corresponding curves of all harmonic function-based methods are given in Figures 3-6 in the Supplemental material. At a specific value of $s$ in the horizontal axis in each of these figures, there is a $\operatorname{MSRE}(K)$ curve where the number of employed moments $|\mathcal{S}(K)|$ 


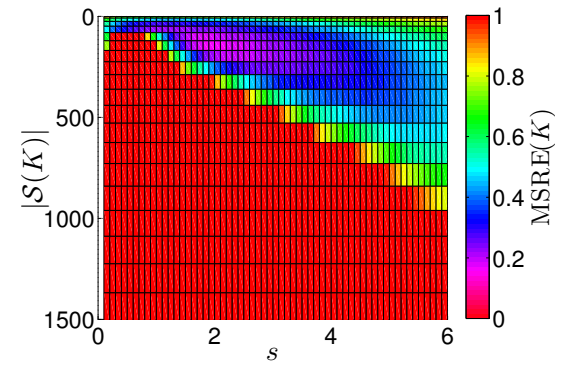

(a) $16 \times 16$

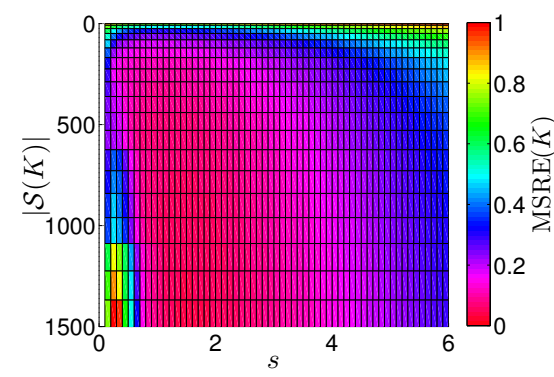

(d) $128 \times 128$

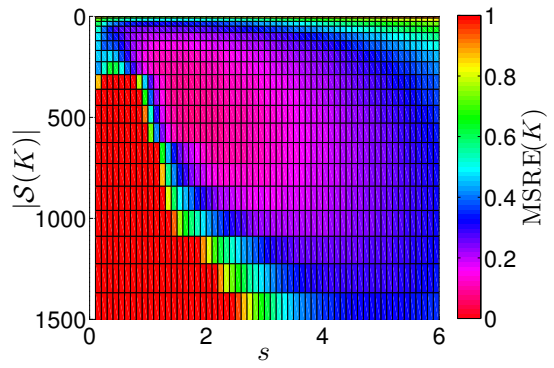

(b) $32 \times 32$

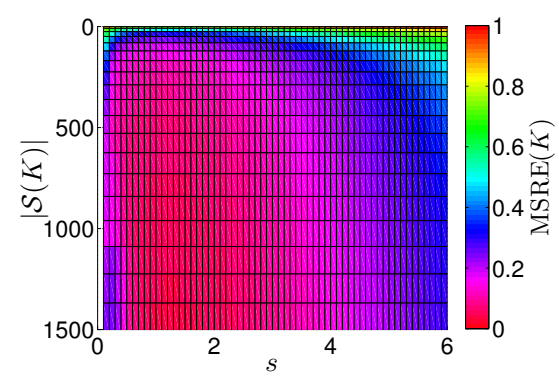

(e) $256 \times 256$

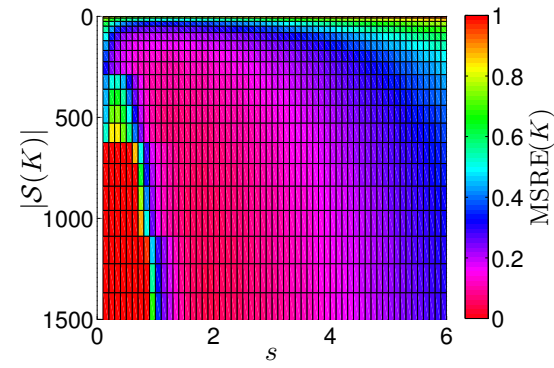

(c) $64 \times 64$

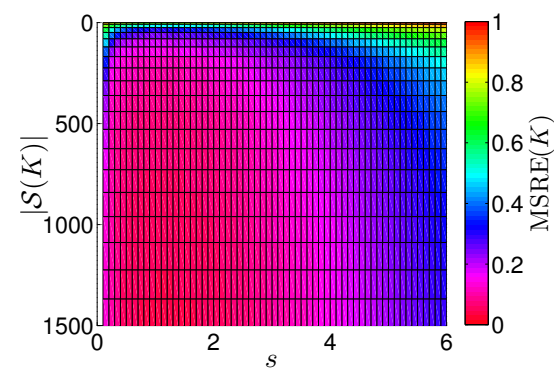

(f) $512 \times 512$

Figure 5: $\operatorname{MSRE}(K)$ curves of GRHFM on the six character datasets at different values of $s$. In each of these figures, at a specific value of $s$ in the horizontal axis, there is a $\operatorname{MSRE}(K)$ curve with the number of employed moments $|\mathcal{S}(K)|$ and $\operatorname{MSRE}(K)$ values illustrated as the ordinate and the color of the grid points having abscissa $s$.

and the mean-square reconstruction error $\operatorname{MSRE}(K)$ are illustrated as the ordinate and the color of the grid points that have abscissa $s$. The values of $\operatorname{MSRE}(K)$ which are outside the color display range $[0,1]$ is assigned the red color. A red color in $\operatorname{MSRE}(K)$ clearly means that the reconstructed image $\hat{f}_{s}$ does not reflect at all $f$. It can be seen from these figures that the color patterns in Supplementary Figure 3 are exactly the same as those in Supplementary Figure 4, suggesting that the reconstructed images by GPCET and GRHFM are the same. This provides experimental evidence for the equivalence between the radial kernels of GPCET and GRHFM that has been disclosed in Section 2. For the purpose of representation and/or compression, GPCET and GRHFM moments can thus be used interchangeably without any change in performance. For this reason, in the remaining of this subsection on image reconstruction and numerical stability, GPCET can be used on behalf of GRHFM in discussions and comparisons with other methods. Among GPCET, GPCT, and GPST, a closer resemblance between the color patterns in Supplementary Figures 3 and 5 is observed. In addition, for a specific image size and at the corresponding abscissas $s$ and ordinates $|\mathcal{S}(K)|, \operatorname{MSRE}(K)$ generally has its highest and lowest values in the case of GPST and GPCT, respectively. This means that GPCT and GPST generally have the highest and lowest representation power, respectively, among harmonic function-based methods. It should be noted that similar observations have also been seen in other applications. For example in compression, it turns out that cosine functions are much more efficient than the other functions.

For each harmonic function-based method and at a specific value of $s$, increasing the image size leads to a decrease in the values of $\operatorname{MSRE}(K)$ at the corresponding ordinates $|\mathcal{S}(K)|$. This means that the reconstructed images $\hat{f}_{s}$ are more similar to the original one $f$. The difference between $\operatorname{MSRE}(K)$ at different image sizes indicates the existence of numerical error in the computed moments. This provides experimental evidence for the effect of image size on this type of error already mentioned in Subsection 4: a smaller image size will lead to a higher numerical error, and vice versa. However, a 


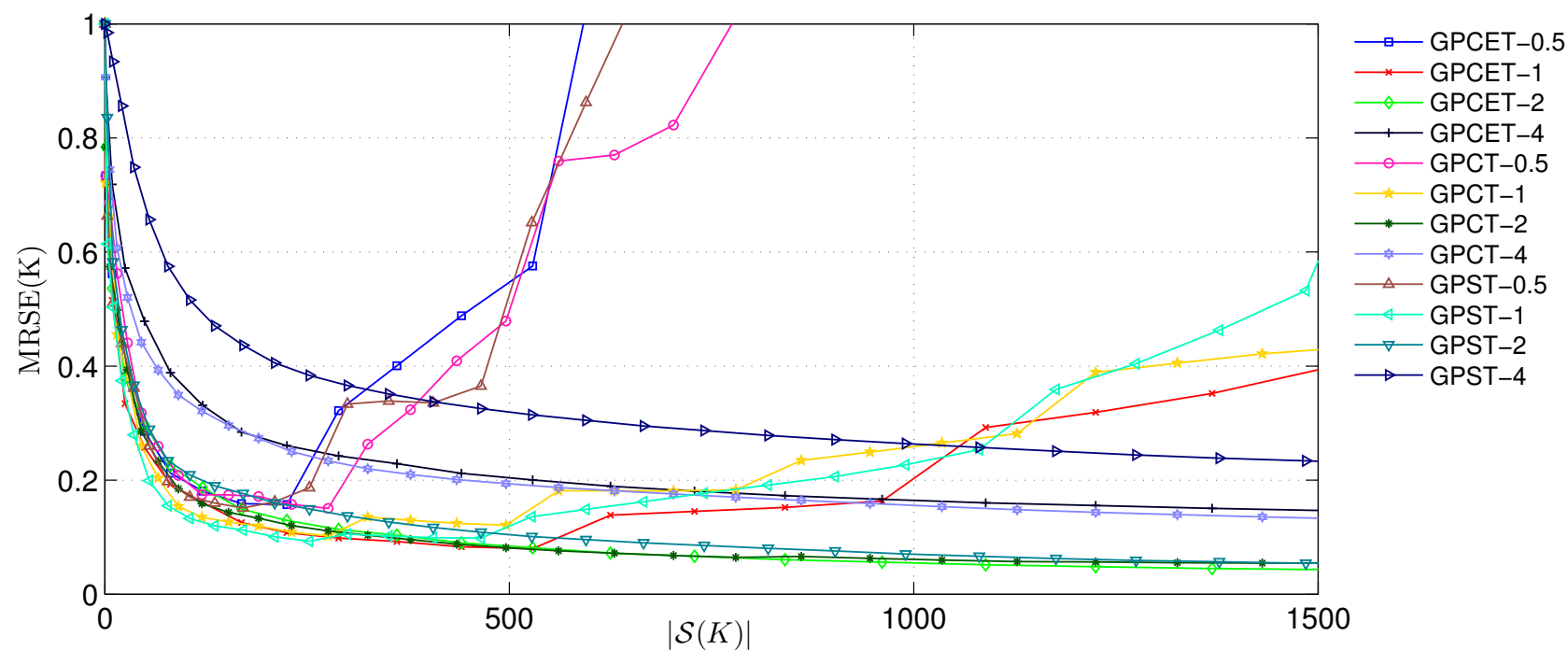

Figure 6: $\operatorname{MSRE}(K)$ curves of harmonic function-based methods (GPCET, GPCT, GPST) at $s=$ $0.5,1,2,4$ on the $64 \times 64$ character dataset.

small difference in $\operatorname{MSRE}(K)$ between image sizes 256 and 512 suggests that the effect of numerical error becomes negligible for large-sized images. In addition, for each harmonic function-based method and at a specific image size, changing the value of $s$ also leads to a change in the values of $\operatorname{MSRE}(K)$ at the corresponding ordinates $|\mathcal{S}(K)|$. The value of $\operatorname{MSRE}(K)$ decreases slowly when $s$ has a too small or a too high value. This is due to the negligence of the extracted moments on certain portions of images as discussed in Subsection 3.2. For better visualization and for the purpose of comparison, Figure 6 illustrates MSRE $(K)$ curves of harmonic function-based methods (GPCET, GPCT, GPST) at $s=0.5,1,2,4$ on the $64 \times 64$ character dataset. These curves are plotted in the traditional 2D Cartesian coordinate system where the number of employed moments $|\mathcal{S}(K)|$ and the mean-square reconstruction error $\operatorname{MSRE}(K)$ are used as the abscissa and ordinate, respectively. The comparison results on the six character datasets are given in Figure 7 in the Supplemental material. From the six figures that correspond to the six character datasets, the above observations on harmonic function-based methods can be verified with relative ease.

Comparison of GPCET with Jacobi polynomial-based and eigenfunction-based methods using $\operatorname{MSRE}(K)$ curves computed from the $64 \times 64$ character dataset is given in Figure 7 . The comparison results on the six character datasets are given in Figure 8 in the Supplemental material. It can be seen from the figure that numerical error causes $\operatorname{MSRE}(K)$ to take higher values at a smaller image size at the corresponding abscissas $|\mathcal{S}(K)|$, similar to the phenomenon already observed in harmonic function-based methods. This provides another experimental evidence for the theoretical arguments on numerical error in Subsection 4.1: a smaller image size will lead to a higher numerical error, and vice versa. Numerical stability of Jacobi polynomial-based methods breaks down when $K$ is increased up to a certain value. The quick deteriorations in the images reconstructed by Jacobi polynomial-based methods observed in Figure 2 in the Supplemental material are exhibited here by sudden upturns in their corresponding $\operatorname{MSRE}(K)$ curves at $K=46,21,23$, and 23 for ZM, PZM, OFMM, and PJFH, respectively. $\operatorname{MSRE}(K)$ curve of CHFM breaks down later at $K=79$ (not shown in the figure). These observations conform with the theoretical arguments on representation error in Subsection 4.2. The starting values of $K$ that cause deteriorations here are equal to the starting radial orders that cause roundoff error in Jacobi polynomial-based methods given in Table 2. For large-sized images, except for GPCET at $s=4$ and for the sudden upturn of Jacobi polynomial-based methods, all comparison 


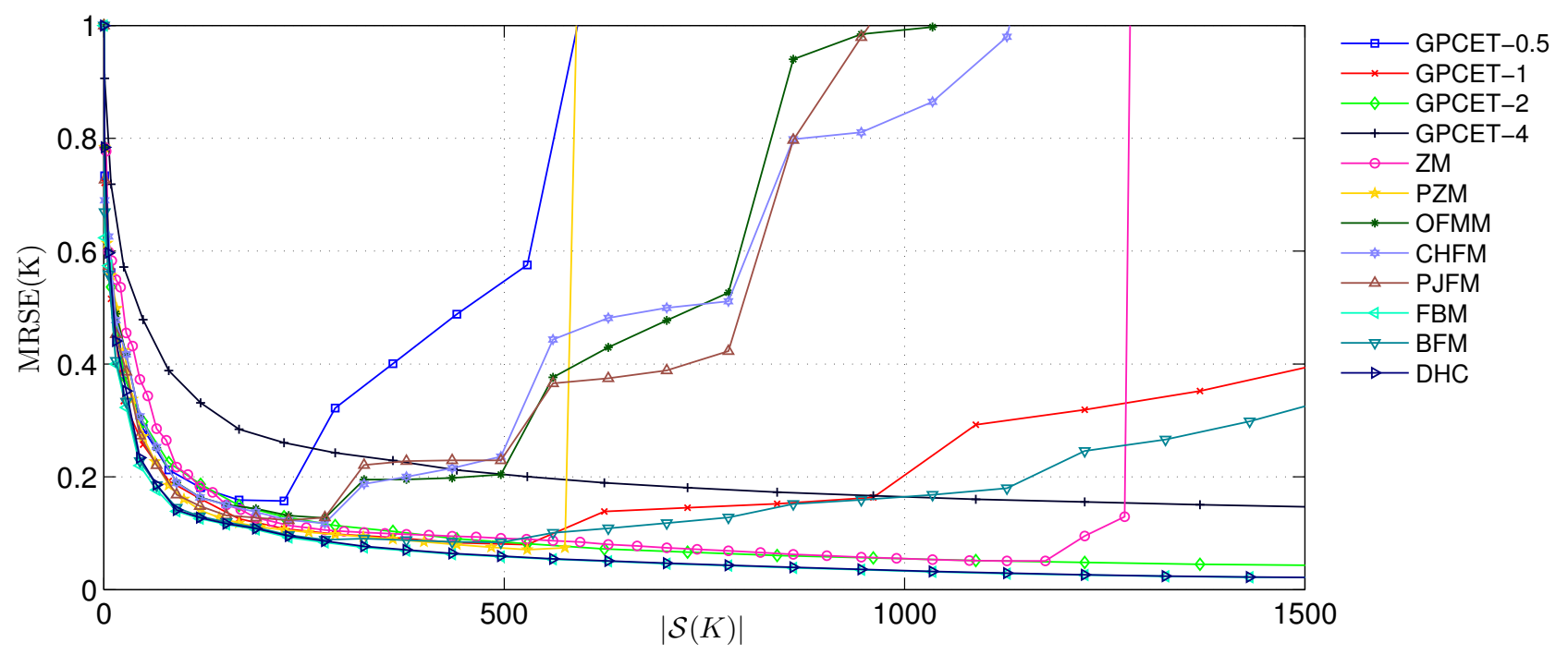

Figure 7: $\operatorname{MSRE}(K)$ curves of GPCET at $s=0.5,1,2,4$, Jacobi polynomial-based (ZM, PZM, OFMM, CHFM, PJFM), and eigenfunction-based (FBM, BFM, DHC) methods on the $64 \times 64$ character dataset.
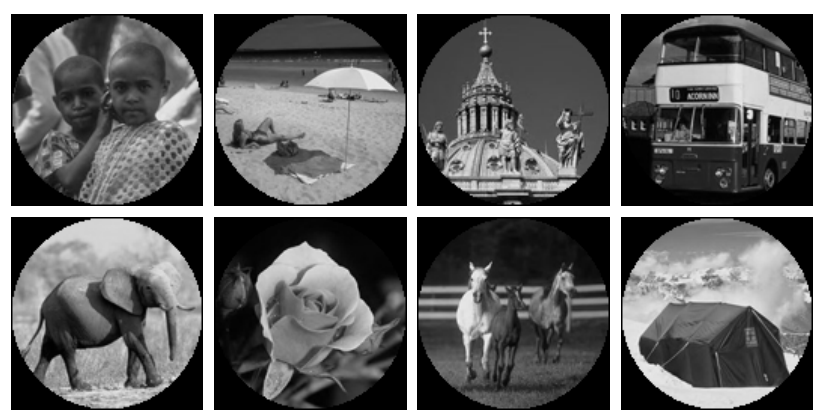

Figure 8: Eight samples out of the 100 images from the COREL photograph dataset used in the pattern recognition experiments.

methods have similar performance with the lowest curves belong to eigenfunction-based methods. For small-sized images, ZM has the highest representation power, followed by GPCET at $s=4$.

It is thus clear from the experiments carried out in this subsection that numerical and representation errors each affects the computed moments in a different way. Approximation error causes a slightly change in the computed moments. On the contrary, a sudden upturn in the $\operatorname{MSRE}(K)$ curve caused by representation error means that the computed moments from that point are totally unreliable and they should not be used in other applications, such as image compression or pattern recognition.

\subsection{Pattern recognition}

In the experiments that follow, images are taken from the COREL photograph dataset [29]: 100 images have been selected, cropped, and scaled to a standard size of $128 \times 128$ pixels. These 100 images are the training images and their computed moments are used as the ground-truth for comparison with those of the testing images. Some samples of these training images are given in Figure 8 where only the pixels $[i, j] \in \mathcal{C}$, with $\mathcal{C}$ defined in Eq. (22), keep their original intensity value. The remaining pixels, which are irrelevant to the experiments, have their intensity value set to zero.

The testing images are generated from the training images by rotating them with angles $\phi=$ $0^{\circ}, 30^{\circ}, \ldots, 330^{\circ}$ and then contaminating them with Gaussian white noise of variances $\sigma^{2}=0.00: 0.05$ : 

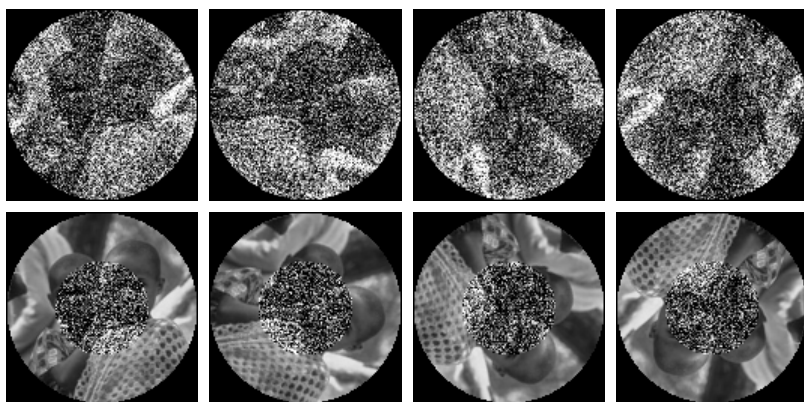

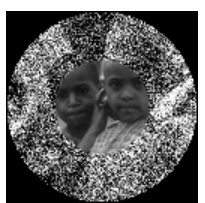

$0^{\circ}$

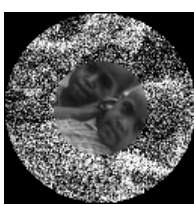

$60^{\circ}$

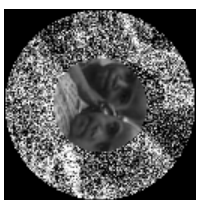

$120^{\circ}$

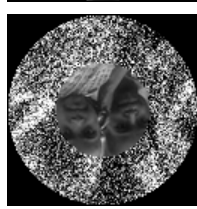

$180^{\circ}$

Figure 9: Sample noisy images of variance $\sigma^{2}=0.1$ at rotating angles $\phi=0^{\circ}, 60^{\circ}, 120^{\circ}, 180^{\circ}$ from the three testing datasets.

0.20 in MATLAB's notation ${ }^{1}$. In order to investigate the role of the parameter $s$ on the recognition results, three different testing datasets (NoiseAll, NoiseInner, and NoiseOuter) are generated separately by restricting the noise to be added to the whole image, the outer portion, and the inner portion, respectively. The inner and outer portions form the whole image and the boundary between them is the circle of radius 32 that has the same center with the image. Thus, for each training image, $12 \times 5 \times 3=180$ testing images are generated from it, making a total of $100 \times 180=18 \times 10^{3}$ images to be classified according to their computed moments. As an example, sample testing images of variance $\sigma^{2}=0.1$ at angles $\phi=0^{\circ}, 60^{\circ}, \ldots, 180^{\circ}$ from these three datasets generated from a single training image are given in Figure 9 .

Each image of the training and testing datasets is then represented by a feature vector, which is the magnitude of its computed moments. Classification is carried out based on the $\ell_{2}$-norm distance between feature vectors. It is not difficult to see that when the testing images are not contaminated by noise, all methods theoretically produce $100 \%$ classification rate on rotation-invariant pattern recognition problems. This is because the magnitude of unit disk-based moments is theoretically invariant to the rotation operation about the origin [13]. However, due to the digital nature of the imagery (sampling and quantization errors) and the numerical computation in digital computers (approximation and representation errors), the computed moments are not truly invariant [30]. For this reason, a set of $K$ values is used on each dataset: $K=3,6,9,12,15$ on NoiseAll, $K=1,2,3,4,5$ on NoiseInner, and $K=2,4,6,8,10$ on NoiseOuter. The reason to use a different set of $K$ values on each dataset is the difference in the amount of discriminative information that remains in the images after adding noise to them. In the presence of noise, the larger the image region that is contaminated by noise is, the less the discriminative information remains in the image. A larger value of $K$ is thus required to maintain the classification performance for images that have a larger noisy region.

The classification rates for harmonic function-based methods (GPCET, GRHFM, GPCT, GPST) at $s=0.5,1,2,4$ on NoiseAll dataset are given in Table 5 . The corresponding results on all the three datasets NoiseAll, NoiseInner, and NoiseOuter are given in Tables 1-3 in the Supplemental material, respectively. From these tables, it can be seen that when the testing images get noisier, meaning an increase in the value of $\sigma^{2}$, the classification rate in the same dataset decreases at the corresponding values of $K$. In addition, the classification rate at the corresponding noise levels $\sigma^{2}$ and in the same

\footnotetext{
${ }^{1}$ The variances are normalized values, corresponding to image's intensity values ranging from 0 to 1 .
} 
dataset increases along with the increase in $K$, or increases when more moments are employed in the feature vector. On NoiseAll, the classification rates of GRHFM, GPCT, GPST have their peak at $s=1$ and decrease as $s$ goes away from 1. In contrast, the classification rate of GPCET does not have a similar trend, it seems to have its minimum value at $s=2$ and increases as $s$ goes away from 2. On NoiseOuter and NoiseInner, as $s$ increases from 0.5 to 4 , the classification rate of all harmonic function-based methods decreases on NoiseOuter and increases on NoiseInner. It should be noted here that when performing on the same dataset, GRHFM generally has the best classification performance at the corresponding values of $K$ and $\sigma^{2}$.

The change in performance that results from a change in the value of $\sigma^{2}$ and $K$ is predictable. The dependence of the performance on the value of $s$ could be explained by the theoretical arguments in Subsection 3.2. The peak performance on NoiseInner/NoiseOuter at $s=4 / s=0.5$ is due to the bias of the distribution of zeros of $R_{n 4}(r) / R_{n 0.5}(r)$ towards $1 / 0$. This means that the information contained in the extracted moments is from the outer/inner portion of the images where the noise is not present. Similarly, the peak performance on NoiseAll of GRHFM, GPCT, GPST at $s=1$ is due to the uniform distribution of zeros of $R_{n 1}(r)$ over $0 \leq r \leq 1$. The irregular trend observed in the classification rates of GPCET could be explained by the complex nature of its radial kernels. Although the zeros of the real and imaginary parts GPCET radial kernels are clearly defined, GPCET radial kernels themselves do not have zeros due to the employed complex exponential functions. And the dominance of GRHFM over other harmonic function-based methods has the following two explanations. First, GRHFM has been shown to be a variant of GPCET in terms of representation, similar to the equivalence between different forms of Fourier series. The radial kernels of GRHFM are trigonometric function-based and do not contain phase information as in the radial kernels of GPCET, which are exponential function-based. Accordingly, GRHFM suffers less from the problem of phase information loss [31] when a magnitude operator is used to compute rotation-invariant feature vectors. As a result, GRHFM generally performs better than GPCET. Second, GPCT and GPST are respectively defined based on the cosine and sine series, which are the so-called half-range expansions of a function. They are special cases of the Fourier series and arise naturally when decomposing an even/odd function. For this reason, many of the properties of cosine and sine series are less elegant and more involved than the corresponding ones of the Fourier series [32]. This may explain for the inferiority of GPCT and GPST to GRHFM in terms of classification performance.

Taking GRHFM as the representative of harmonic function-based methods, comparison of GRHFM at $s=0.5,1,2,4$ with non-orthogonal (ART, GFD, RM), Jacobi polynomial-based (ZM, PZM, OFMM, CHFM, PJFM), and eigenfunction-based (FBM, BFM, DHC) methods on NoiseAll, NoiseInner, and NoiseOuter datasets are given in Tables 4-6 in the Supplemental material, respectively. Besides similar trends in the dependence of the classification rate on the values of $K$ and $\sigma^{2}$, it can also be seen from these tables that non-orthogonal methods have lower classification rates than orthogonal ones on the three datasets. This inferiority demonstrates clearly that non-orthogonal methods are less efficient than orthogonal ones in rotation-invariant pattern recognition problems. In addition, except for OFMM on NoiseAll, Jacobi polynomial-based methods have lower performance than GRHFM at their peak performance ( $s=1$ on NoiseAll, $s=4$ on NoiseInner, and $s=0.5$ on NoiseOuter) at the corresponding values of $K$ and $\sigma^{2}$ and in the same dataset. Eigenfunction-based methods perform better than GRHFM on NoiseAll $(s=1)$ when the value of $K$ is high enough $K \geq 6$. They perform worse than GRHFM on NoiseInner $(s=4)$ and have comparable performance with GRHFM on NoiseOuter $(s=0.5)$.

From the experimental results on pattern recognition, it can be concluded that harmonic functionbased moments could be used as region-based feature vector in rotation-invariant pattern recognition problems. They out-perform non-orthogonal and Jacobi polynomial-based moments and have comparable 


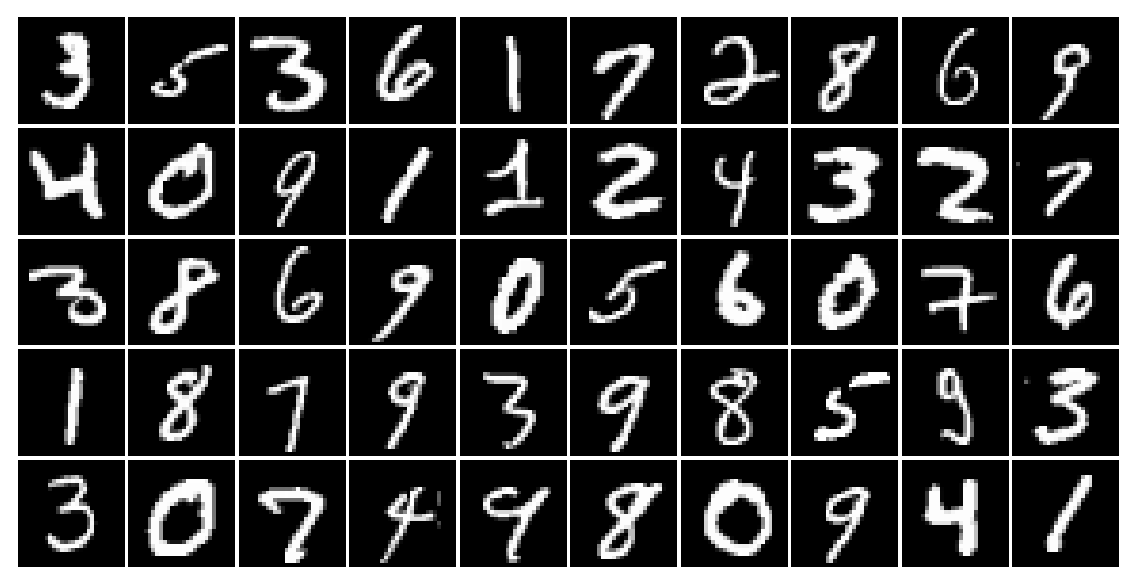

Figure 10: Sample character images from the MNIST dataset.

performance with eigenfunction-based moments on the three experiential datasets. Moreover, the decisive role of $s$ on the recognition results, as theoretically argued in Subsection 3.2, has also been confirmed.

The performance of GRHFM has also been evaluated on the well-known MNIST dataset [33] of handwritten digits. MNIST consists of 60,000 training and 10,000 testing images of size $28 \times 28$ pixels. In this experiment, only 6000 images are used for training and 1000 for testing to avoid large memory requirement. Some sample digits are shown in Figure 10. To further demonstrate the benefit of harmonic function-based moments and to simulate the imperfectness in digit image normalization, the images in the testing set are randomly rotated by $-45^{\circ}$ to $45^{\circ}$. In the following comparison, the "direct" method uses raw pixel intensities as feature vector for the nearest neighbor classifier. GPCET, GRHFM, GPCT, and GPST moments are combined into a single feature vector to make it more discriminant. This is because harmonic function-based moments capture different characteristics of an image. The classification results are shown in Table 6. It can be seen from the table that harmonic function-based moments give relatively high classification accuracy, while the direct method fails as expected. These results also demonstrate clearly the benefit of rotation-invariant features in a real-world pattern recognition problem. Harmonic function-based moments extracted from the images do contain adequate information for pattern classification. However, it should be noted here that although harmonic function-based moments already provide a sufficient set of features for image representation, these features are not specifically designed to for classification. This is because the discrimination power is not considered from the beginning. Thus, a feature selection procedure combined with an intelligent classifier might be necessary in order to take full advantage of the rich representation capability of harmonic function-based moments.

\section{Conclusions}

In this paper, the generalization of existing unit disk-based orthogonal moments using harmonic functions has been pursued where the radial kernels are defined using exponential or trigonometric functions (GPCET or GRHFM), cosine series (GPCT), and sine series (GPST). The sets of harmonic functionbased orthogonal kernels have been shown to be complete in the Hilbert space of square-integrable continuous complex-valued functions. In addition, the use of a parameter $s$ in the definition brings in four classes of moments that maintain beneficial properties of the original moments (PCET, RHFM, PCT, and PST) while giving more flexibility in their definition. This flexibility has been demonstrated to be useful both theoretically and experimentally in some particular applications, especially in image 
compression and pattern recognition problems.

In terms of representation capability, harmonic function-based methods suffer from numerical error, like all other methods. However, they do not suffer from representation error as Jacobi polynomial-based methods do. As a result, the well-known numerical instability in Jacobi polynomial-based methods does not exist in harmonic function-based methods. Apart from this numerical instability, the representation power of all unit disk-based orthogonal moments is comparable. However, the ability to control the representation emphasis on certain image regions by changing the value of $s$ is a distinct feature of harmonic function-based methods. It is possible to have a faster reconstruction of the image function in certain regions of interest. This characteristic could lead to potential applications in image compression.

In rotation-invariant pattern recognition problems, harmonic function-based methods have been shown to generally perform better than non-orthogonal and Jacobi polynomial-based methods. They also have comparable performance with eigenfunction-based methods. Thus, harmonic function-based moments could be used as region-based feature vector in rotation-invariant pattern recognition problems. In addition, the decisive role of $s$ on the recognition results has been confirmed. It can be used to direct the extracted feature vector to emphasize on certain image regions that contain discriminative information. This ability is also a distinct feature of harmonic function-based methods.

Since harmonic function-based moments can be computed very quickly by exploiting the recurrence relations among complex exponentials and trigonometric functions [23], they promise to provide an efficient and useful technique for a number of image processing and pattern recognition applications.

\section{References}

[1] M. R. Teague, "Image analysis via the general theory of moments," Journal of the Optical Society of America, vol. 70, no. 8, pp. 920-930, 1980.

[2] A. B. Bhatia and E. Wolf, "On the circle polynomials of Zernike and related orthogonal sets," Mathematical Proceedings of the Cambridge Philosophical Society, vol. 50, pp. 40-48, 1954.

[3] C.-H. Teh and R. T. Chin, "On image analysis by the methods of moments," IEEE Transactions on Pattern Analysis and Machine Intelligence, vol. 10, no. 4, pp. 496-513, 1988.

[4] Y. S. Abu-Mostafa and D. Psaltis, "Recognitive aspects of moment invariants," IEEE Transactions on Pattern Analysis and Machine Intelligence, vol. 6, no. 6, pp. 698-706, 1984.

[5] D. Zhang and G. Lu, "Shape-based image retrieval using generic Fourier descriptor," Signal Processing: Image Communication, vol. 17, no. 10, pp. 825-848, 2002.

[6] M. Bober, F. Preteux, and W.-Y. Y. Kim, "Shape descriptors," in Introduction to MPEG 7: Multimedia Content Description Language, B. S. Manjunat, P. Salembier, and T. Sikora, Eds. John Wiley \& Sons, 2002, pp. 231-260.

[7] T. H. Koornwinder, R. Wong, R. Koekoek, and R. F. Swarttouw, "Orthogonal polynomials," in NIST Handbook of Mathematical Functions, F. W. J. Olver, D. W. Lozier, R. F. Boisvert, and C. W. Clark, Eds. Cambridge University Press, 2010, ch. 15, pp. 435-484.

[8] F. Zernike, "Beugungstheorie des schneidenver-fahrens und seiner verbesserten form, der phasenkontrastmethode," Physica, vol. 1, no. 7-12, pp. 689 - 704, 1934.

[9] Y. Sheng and L. Shen, "Orthogonal Fourier-Mellin moments for invariant pattern recognition," Journal of the Optical Society of America A, vol. 11, no. 6, pp. 1748-1757, 1994. 
[10] Z. Ping, R. Wu, and Y. Sheng, "Image description with Chebyshev-Fourier moments," Journal of the Optical Society of America A, vol. 19, no. 9, pp. 1748-1754, 2002.

[11] G. Amu, S. Hasi, X. Yang, and Z. Ping, "Image analysis by pseudo-Jacobi $(\mathrm{p}=4, \mathrm{q}=3)$-Fourier moments," Applied Optics, vol. 43, no. 10, pp. 2093-2101, 2004.

[12] J. Flusser, T. Suk, and B. Zitová, Moments and Moment Invariants in Pattern Recognition. John Wiley \& Sons, 2009.

[13] T. V. Hoang, "Image Representations for Pattern Recognition," Ph.D. dissertation, University of Nancy, December 2011.

[14] Z. Ping, H. Ren, J. Zou, Y. Sheng, and W. Bo, "Generic orthogonal moments: Jacobi-Fourier moments for invariant image description," Pattern Recognition, vol. 40, no. 4, pp. 1245-1254, 2007.

[15] T. V. Hoang and S. Tabbone, "Errata and comments on "Generic orthogonal moments: JacobiFourier moments for invariant image description"," Pattern Recognition, vol. 46, no. 11, pp. 3148-3155, 2013.

[16] F. Bowman, Introduction to Bessel Functions. Dover Publications, 1958.

[17] Q. Wang, O. Ronneberger, and H. Burkhardt, "Rotational invariance based on Fourier analysis in polar and spherical coordinates," IEEE Transactions on Pattern Analysis and Machine Intelligence, vol. 31, no. 9, pp. 1715-1722, 2009.

[18] S. Guan, C.-H. Lai, and G. W. Wei, "Fourier-Bessel analysis of patterns in a circular domain," Physica D: Nonlinear Phenomena, vol. 151, no. 2-4, pp. 83-98, 2001.

[19] B. Xiao, J.-F. Ma, and X. Wang, "Image analysis by Bessel-Fourier moments," Pattern Recognition, vol. 43 , no. 8 , pp. 2620-2629, 2010.

[20] S. C. Verrall and R. Kakarala, "Disk-harmonic coefficients for invariant pattern recognition," Journal of the Optical Society of America A, vol. 15, no. 2, pp. 389-401, 1998.

[21] H. Ren, Z. Ping, W. Bo, W. Wu, and Y. Sheng, "Multidistortion-invariant image recognition with radial harmonic Fourier moments," Journal of the Optical Society of America A, vol. 20, no. 4, pp. 631-637, 2003.

[22] P.-T. Yap, X. Jiang, and A. C. Kot, "Two-dimensional polar harmonic transforms for invariant image representation," IEEE Transactions on Pattern Analysis and Machine Intelligence, vol. 32, no. 6, pp. 1259-1270, 2010.

[23] T. V. Hoang and S. Tabbone, "Fast computation of orthogonal polar harmonic transforms," in Proceedings of the 21th International Conference on Pattern Recognition, 2012, pp. 3160-3163.

[24] — , "The generalization of the $R$-transform for invariant pattern representation," Pattern Recognition, vol. 45, no. 6, pp. 2145-2163, 2012.

[25] — - "Generic polar harmonic transforms for invariant image description," in Proceedings of the 18th IEEE International Conference on Image Processing, 2011, pp. 845-848.

[26] C. Fefferman, "On the convergence of multiple Fourier series," Bulletin of the American Mathematical Society, vol. 77, no. 5, pp. 744-745, 1971. 
[27] S. X. Liao and M. Pawlak, "On the accuracy of Zernike moments for image analysis," IEEE Transactions on Pattern Analysis and Machine Intelligence, vol. 20, no. 12, pp. 1358-1364, 1998.

[28] G. A. Papakostas, Y. S. Boutalis, C. Papaodysseus, and D. K. Fragoulis, "Numerical error analysis in Zernike moments computation," Image and Vision Computing, vol. 24, no. 9, pp. 960-969, 2006.

[29] J. Z. Wang, J. Li, and G. Wiederhold, "SIMPLIcity: semantics-sensitive integrated matching for picture libraries," IEEE Transactions on Pattern Analysis and Machine Intelligence, vol. 23, no. 9, pp. 947-963, 2001.

[30] C.-H. Teh and R. T. Chin, "On digital approximation of moment invariants," Computer Vision, Graphics, and Image Processing, vol. 33, no. 3, pp. 318-326, 1986.

[31] A. Khotanzad and Y. H. Hong, "Invariant image recognition by Zernike moments," IEEE Transactions on Pattern Analysis and Machine Intelligence, vol. 12, no. 5, pp. 489-497, 1990.

[32] P. Yip, "Sine and cosine transforms," in Transforms and Applications Handbook, 3rd ed., A. D. Poularikas, Ed. CRC Press, 2010, ch. 3.

[33] Y. LeCun, L. Bottou, Y. Bengio, and P. Haffner, "Gradient-based learning applied to document recognition," Proceedings of the IEEE, vol. 86, no. 11, pp. 2278-2324, 1998. 


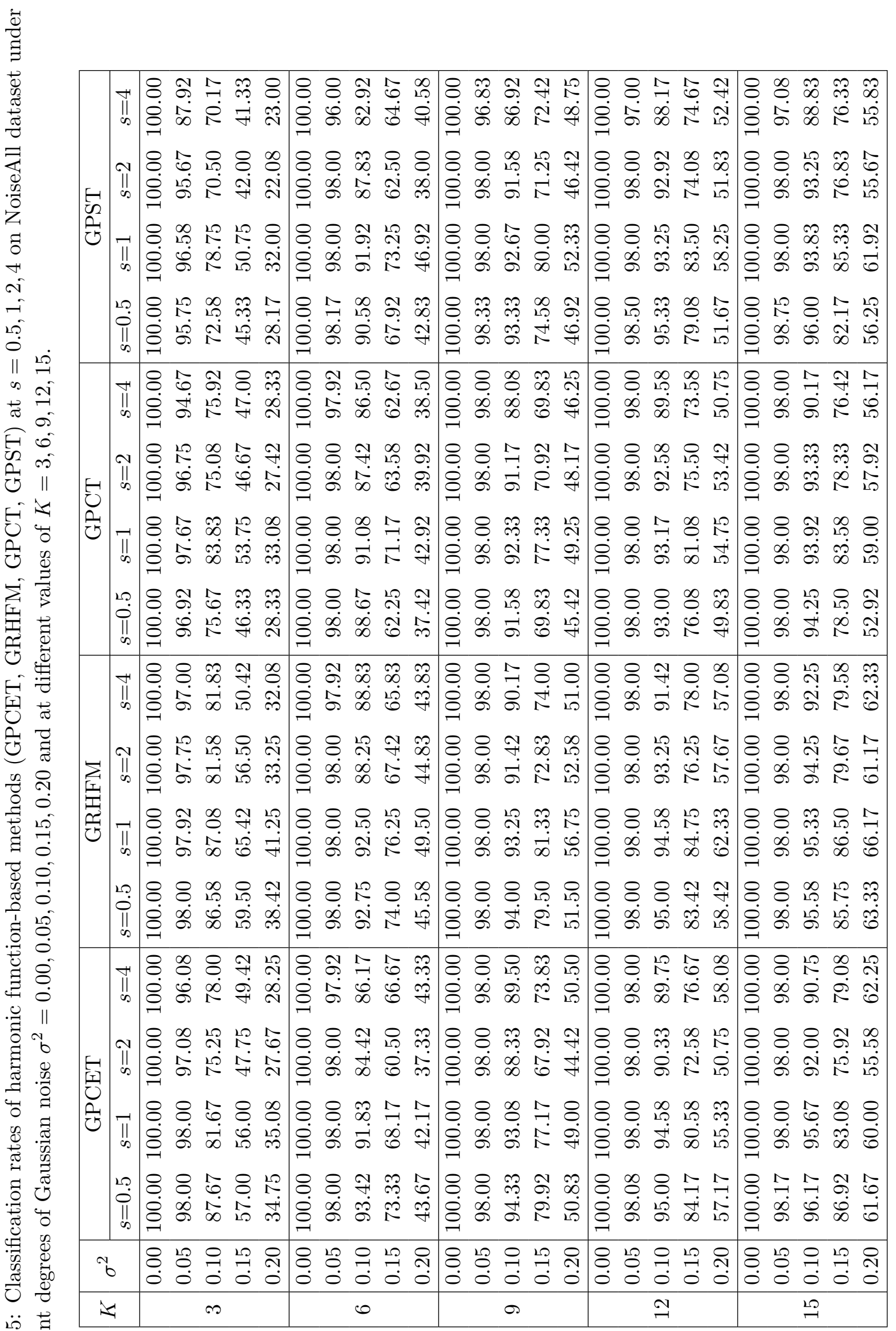


Table 6: Classification rates of "direct" and harmonic function-based methods at $s=2$ using the MNIST dataset.

\begin{tabular}{lcc}
\hline \hline & Direct & Harmonic moments \\
\hline Without rotation & 89.20 & 96.54 \\
With rotation & 16.49 & 91.19 \\
\hline
\end{tabular}




\author{
Supplemental Material to \\ "Generic Polar Harmonic Transforms for \\ Invariant Image Representation" \\ Thai V. Hoang*,1 and Salvatore Tabbone ${ }^{2}$ \\ ${ }^{1}$ Inria Nancy-Grand Est, 54600 Villers-lès-Nancy, France \\ ${ }^{2}$ Université de Lorraine, LORIA, 54506 Vandouvre-lès-Nancy, France
}

\footnotetext{
${ }^{*}$ Corresponding author

E-mail address: vanthai.hoang@inria.fr, Telephone: +33 3549585 60, Fax: +33 383278319 .
} 


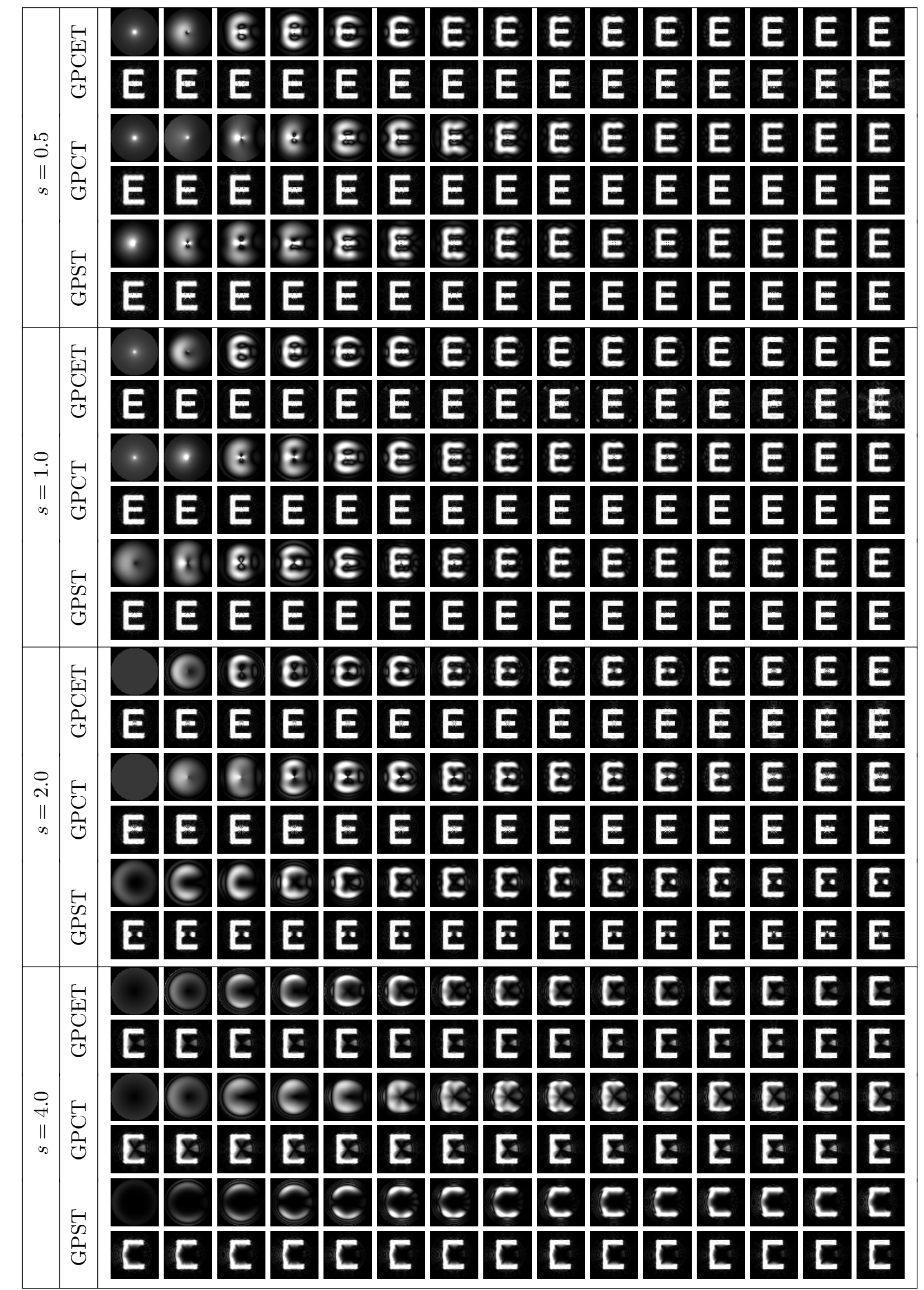

Figure 1: Some samples of reconstructed images from the character image "E" of size $64 \times 64$ pixels by harmonic function-based methods at $s=0.5,1,2,4$ for $K=0,1, \ldots, 29$ (GPCET, GPCT) and $K=1,2, \ldots, 30$ (GPST) (from left to right, top to bottom). 


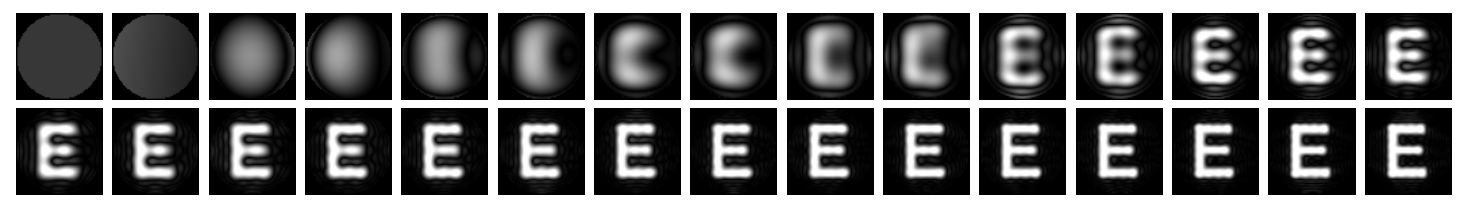

(a) ZM

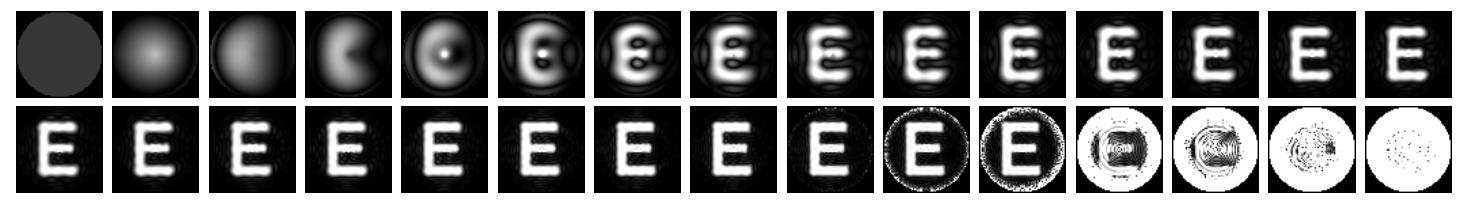

(b) PZM

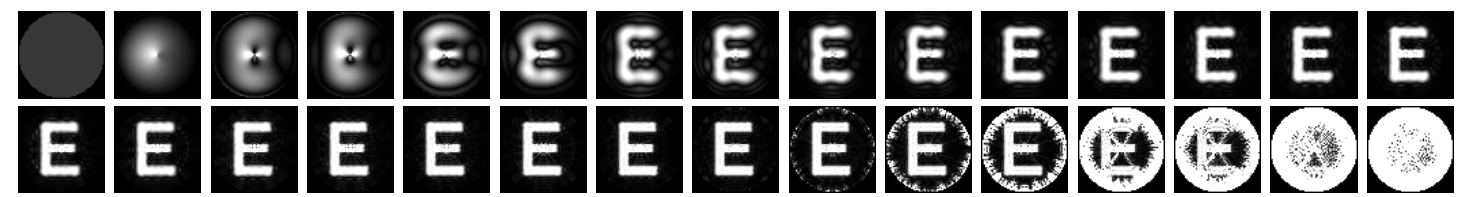

(c) OFMM

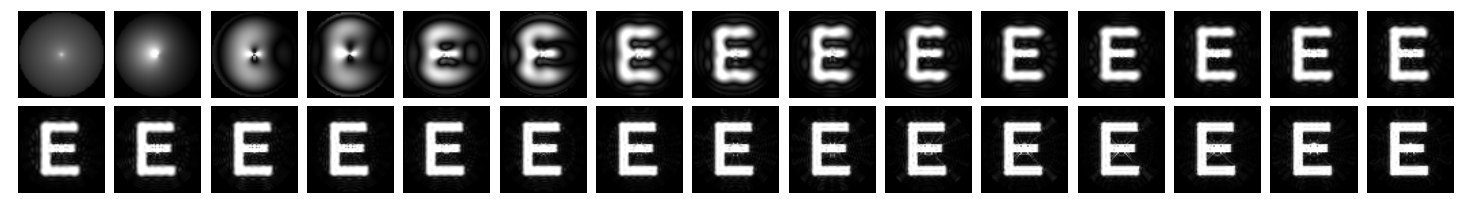

(d) CHFM

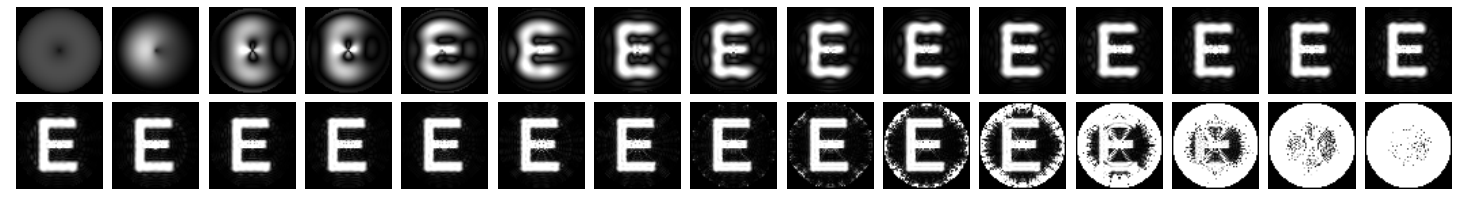

(e) PJFM

\begin{tabular}{l|l|l|l|l|l|l|l|l|l|l|l|l|l|l|}
\hline D & & E & E & E & E & $\mathrm{E}$ & $\mathrm{E}$ & $\mathrm{E}$ & $\mathrm{E}$ & $\mathrm{E}$ & $\mathrm{E}$ & $\mathrm{E}$ & $\mathrm{E}$ & $\mathrm{E}$ \\
\hline $\mathrm{E}$ & $\mathrm{E}$ & $\mathrm{E}$ & $\mathrm{E}$ & $\mathrm{E}$ & $\mathrm{E}$ & $\mathrm{E}$ & $\mathrm{E}$ & $\mathrm{E}$ & $\mathrm{E}$ & $\mathrm{E}$ & $\mathrm{E}$ & $\mathrm{E}$ & $\mathrm{E}$ & $\mathrm{E}$ \\
\hline
\end{tabular}

(f) FBM

\begin{tabular}{l|l|l|l|l|l|l|l|l|l|l|l|l|l|l|}
\hline & $\mathrm{C}$ & $\mathrm{E}$ & $\mathrm{E}$ & $\mathrm{E}$ & $\mathrm{E}$ & $\mathrm{E}$ & $\mathrm{E}$ & $\mathrm{E}$ & $\mathrm{E}$ & $\mathrm{E}$ & $\mathrm{E}$ & $\mathrm{E}$ & $\mathrm{E}$ & $\mathrm{E}$ \\
\hline $\mathrm{E}$ & $\mathrm{E}$ & $\mathrm{E}$ & $\mathrm{E}$ & $\mathrm{E}$ & $\mathrm{E}$ & $\mathrm{E}$ & $\mathrm{E}$ & $\mathrm{E}$ & $\mathrm{E}$ & $\mathrm{E}$ & $\mathrm{E}$ & $\mathrm{E}$ & $\mathrm{E}$ & $\mathrm{E}$ \\
\hline
\end{tabular}

(g) BFM

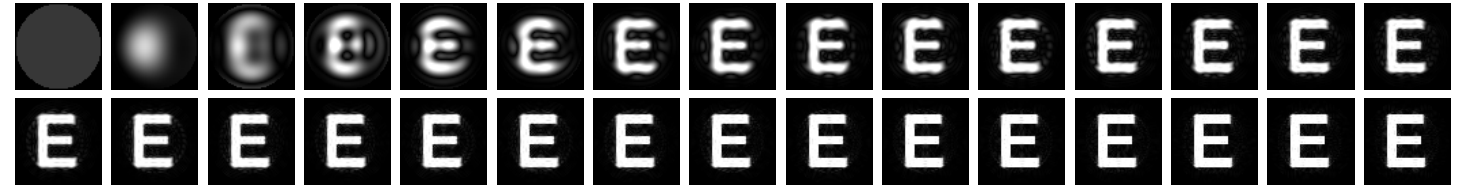

(h) DHC

Figure 2: Some samples of reconstructed images from the character image "E" of size $64 \times 64$ pixels by Jacobi polynomial-based (ZM, PZM, OFMM, CHFM, PJFM) and eigenfunction-based (FBM, BFM, DHC) methods for $K=0,1, \ldots, 29$ (from left to right, top to bottom). 


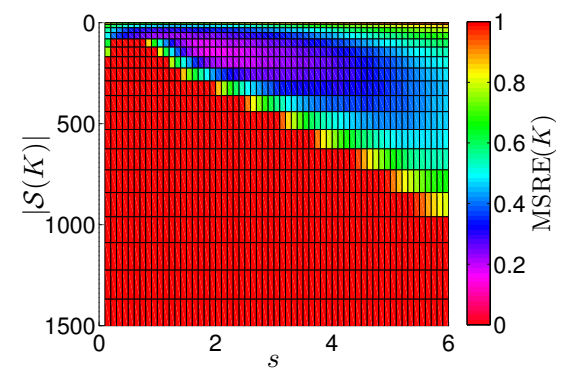

(a) $16 \times 16$

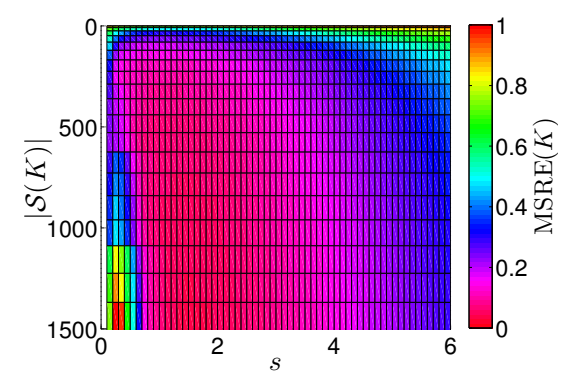

(d) $128 \times 128$

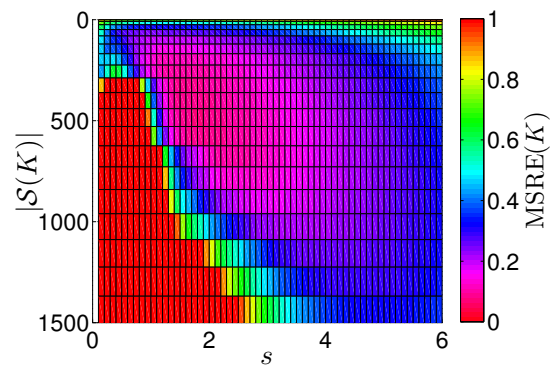

(b) $32 \times 32$

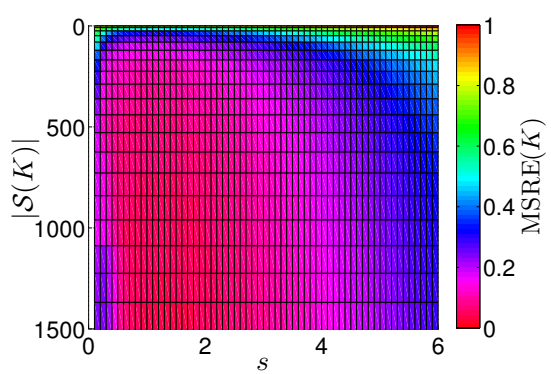

(e) $256 \times 256$

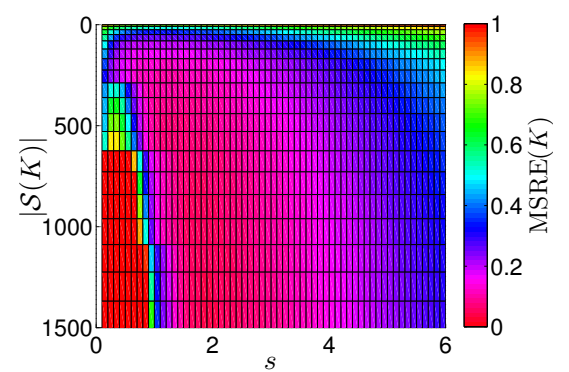

(c) $64 \times 64$

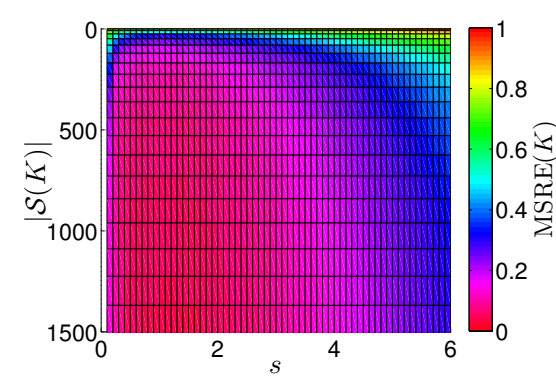

(f) $512 \times 512$

Figure 3: $\operatorname{MSRE}(K)$ curves of GPCET on the six character datasets at different values of $s$. In each of these figures, at a specific value of $s$ in the horizontal axis, there is a $\operatorname{MSRE}(K)$ curve with the number of employed moments $|\mathcal{S}(K)|$ and $\operatorname{MSRE}(K)$ values illustrated as the ordinate and the color of the grid points having abscissa $s$.

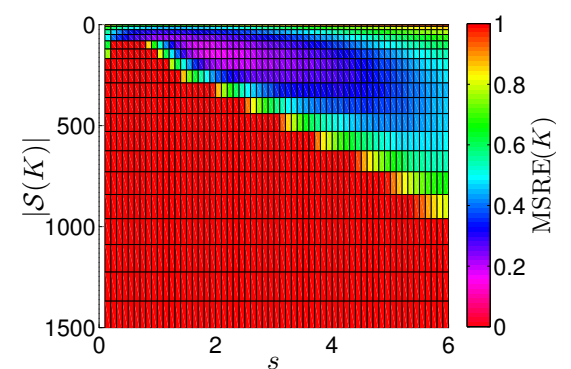

(a) $16 \times 16$

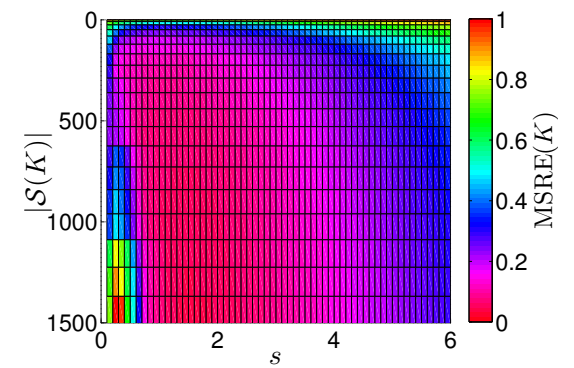

(d) $128 \times 128$

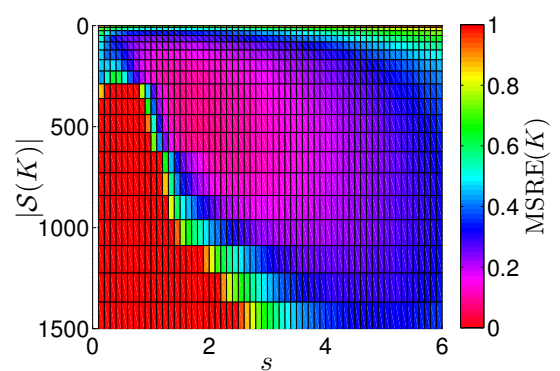

(b) $32 \times 32$

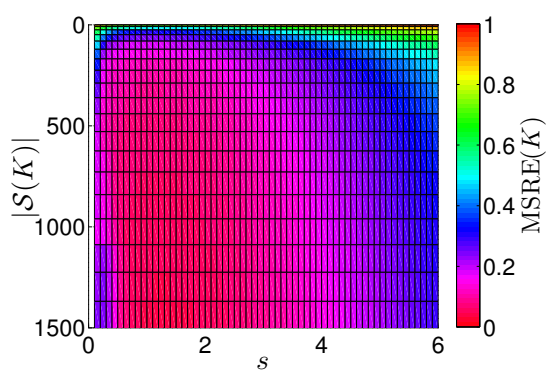

(e) $256 \times 256$

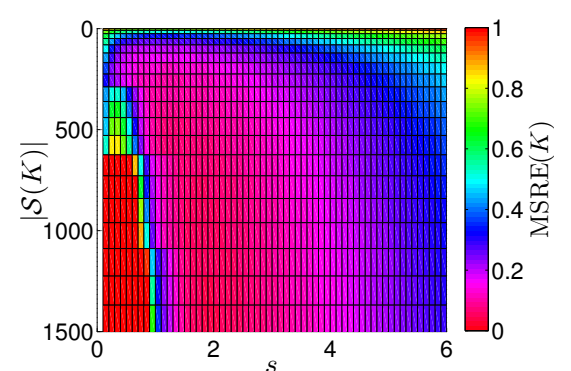

(c) $64 \times 64$

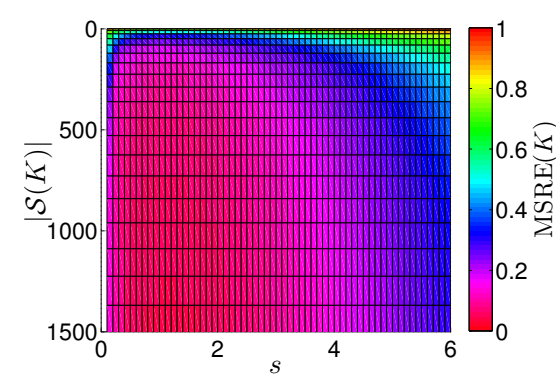

(f) $512 \times 512$

Figure 4: $\operatorname{MSRE}(K)$ curves of GRHFM on the six character datasets at different values of $s$. In each of these figures, at a specific value of $s$ in the horizontal axis, there is a $\operatorname{MSRE}(K)$ curve with the number of employed moments $|\mathcal{S}(K)|$ and $\operatorname{MSRE}(K)$ values illustrated as the ordinate and the color of the grid points having abscissa $s$. 


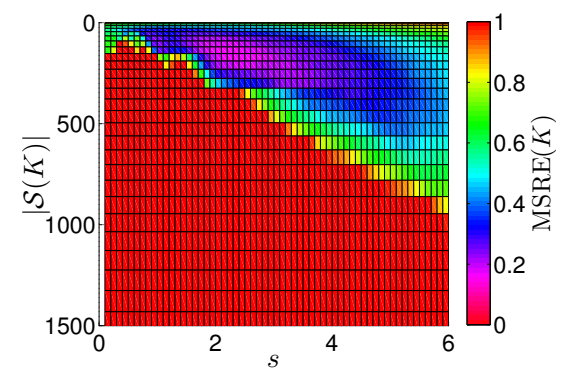

(a) $16 \times 16$

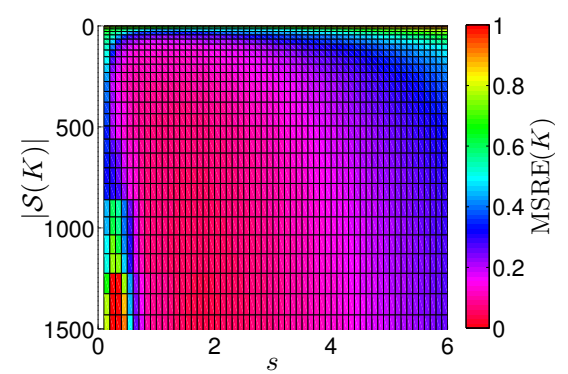

(d) $128 \times 128$

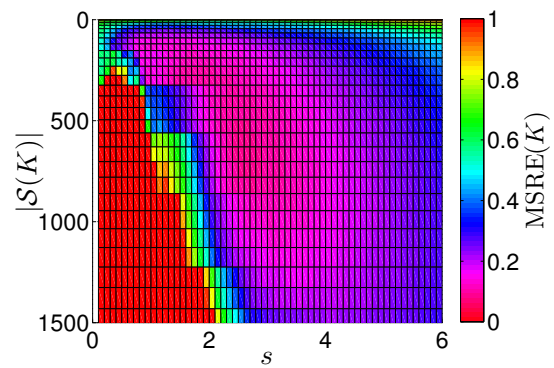

(b) $32 \times 32$

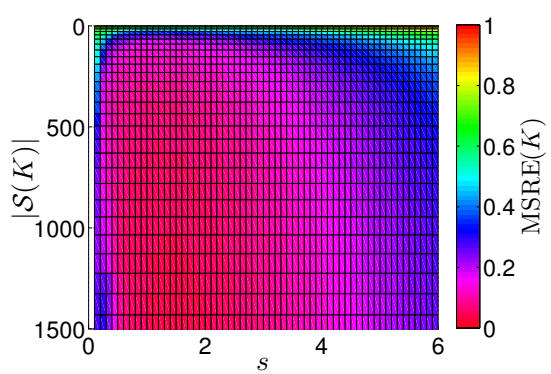

(e) $256 \times 256$

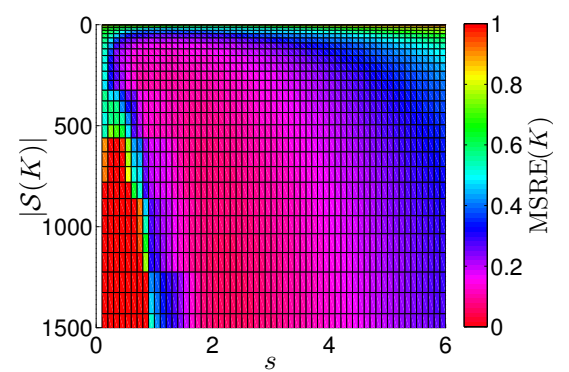

(c) $64 \times 64$

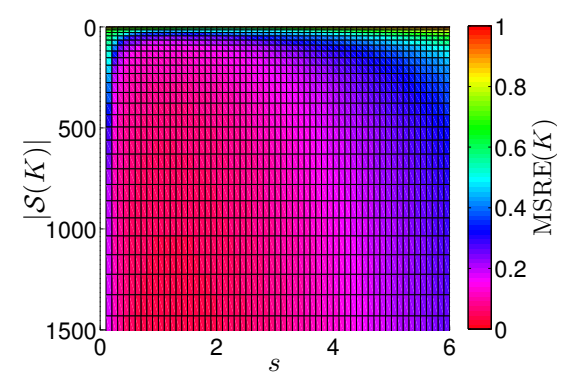

(f) $512 \times 512$

Figure 5: $\operatorname{MSRE}(K)$ curves of GPCT on the six character datasets at different values of $s$. In each of these figures, at a specific value of $s$ in the horizontal axis, there is a $\operatorname{MSRE}(K)$ curve with the number of employed moments $|\mathcal{S}(K)|$ and $\operatorname{MSRE}(K)$ values illustrated as the ordinate and the color of the grid points having abscissa $s$.

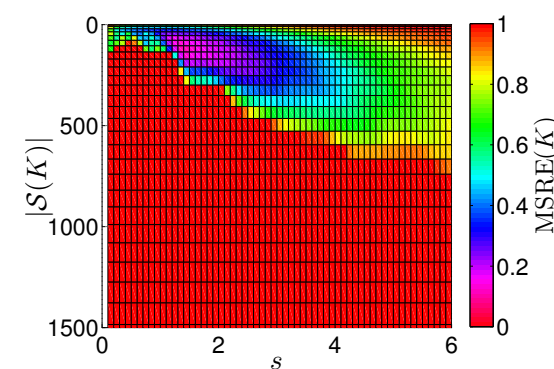

(a) $16 \times 16$

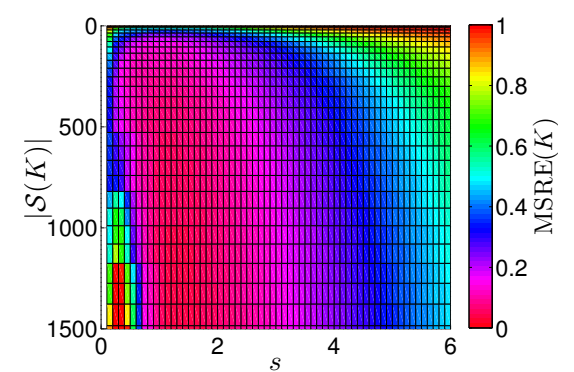

(d) $128 \times 128$

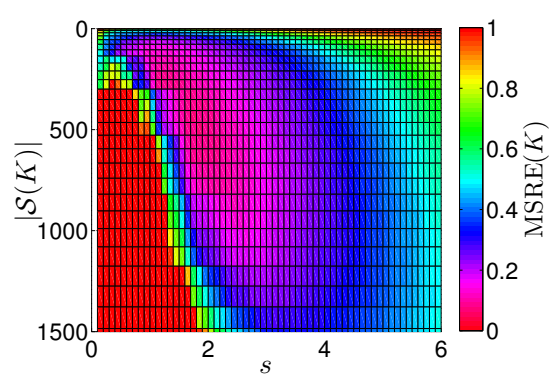

(b) $32 \times 32$

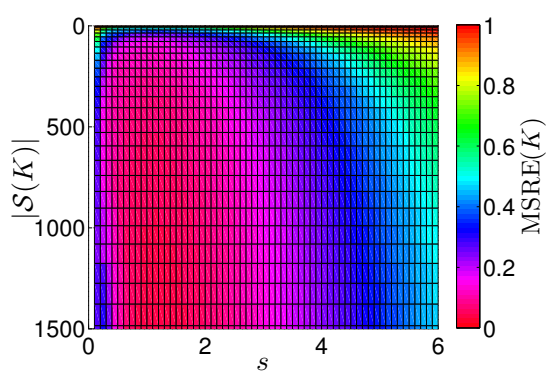

(e) $256 \times 256$

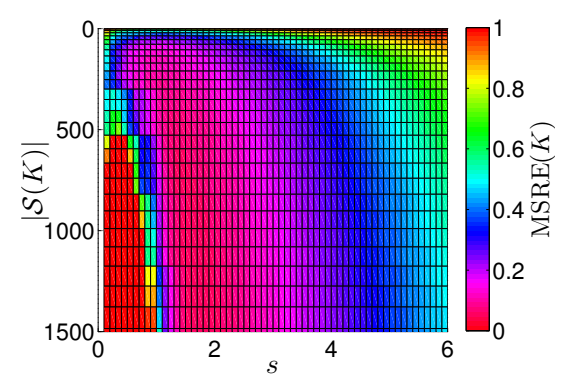

(c) $64 \times 64$

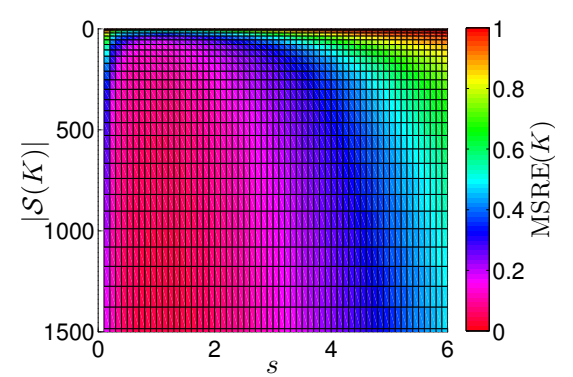

(f) $512 \times 512$

Figure 6: $\operatorname{MSRE}(K)$ curves of GPST on the six character datasets at different values of $s$. In each of these figures, at a specific value of $s$ in the horizontal axis, there is a $\operatorname{MSRE}(K)$ curve with the number of employed moments $|\mathcal{S}(K)|$ and $\operatorname{MSRE}(K)$ values illustrated as the ordinate and the color of the grid points having abscissa $s$. 


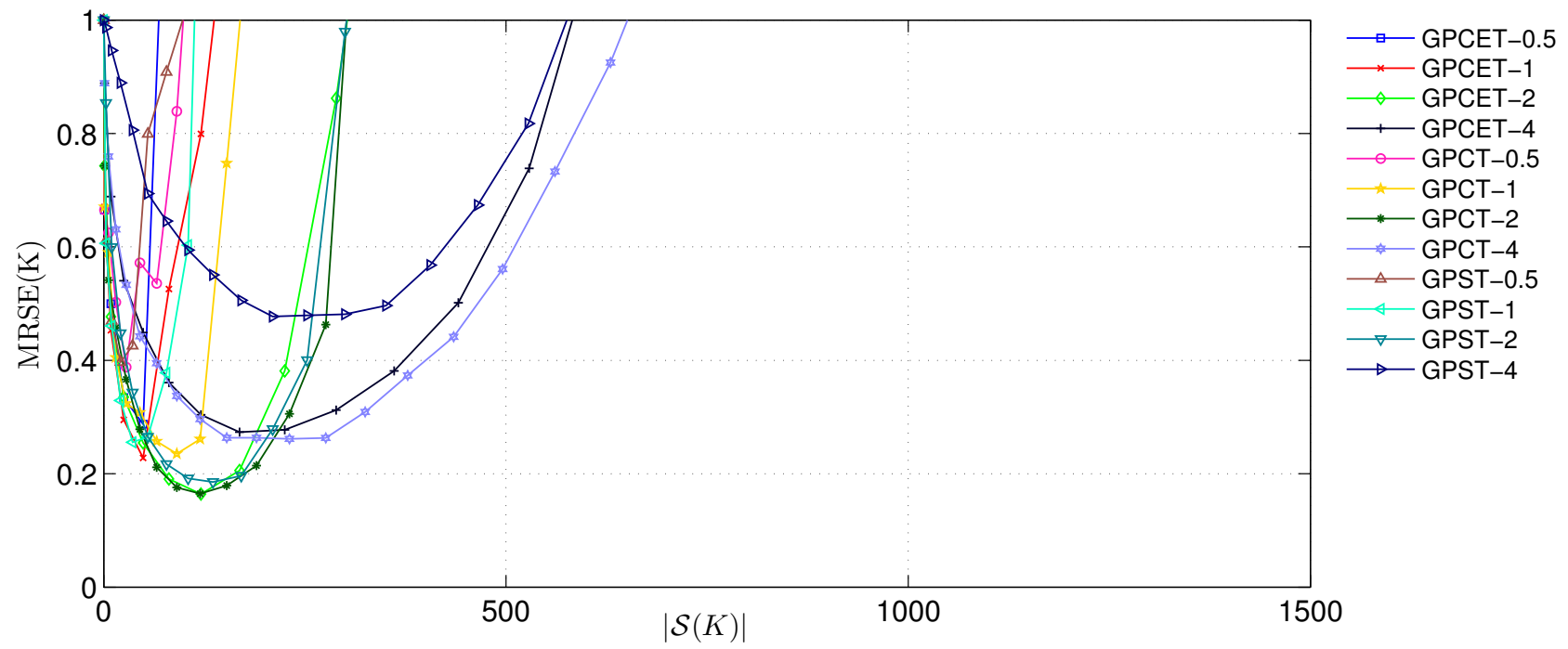

(a) $16 \times 16$

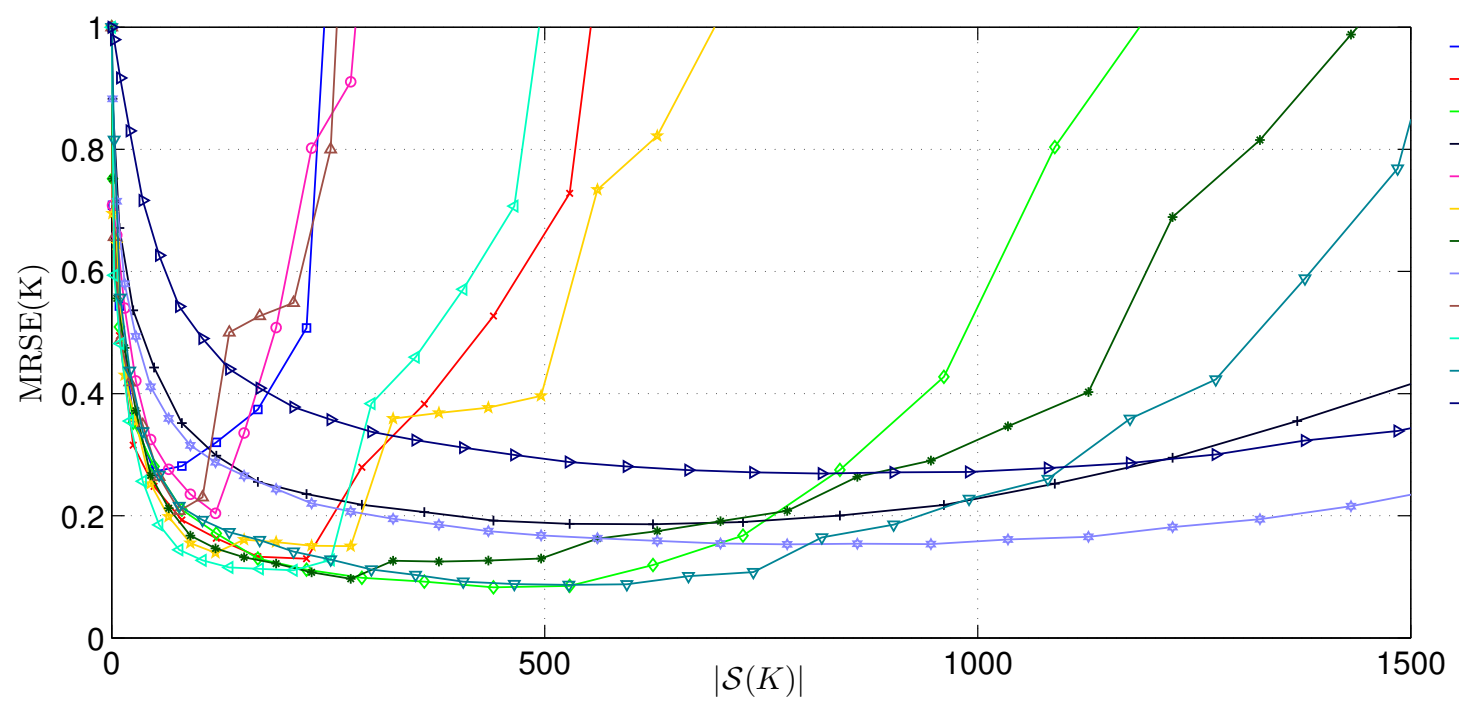

(b) $32 \times 32$
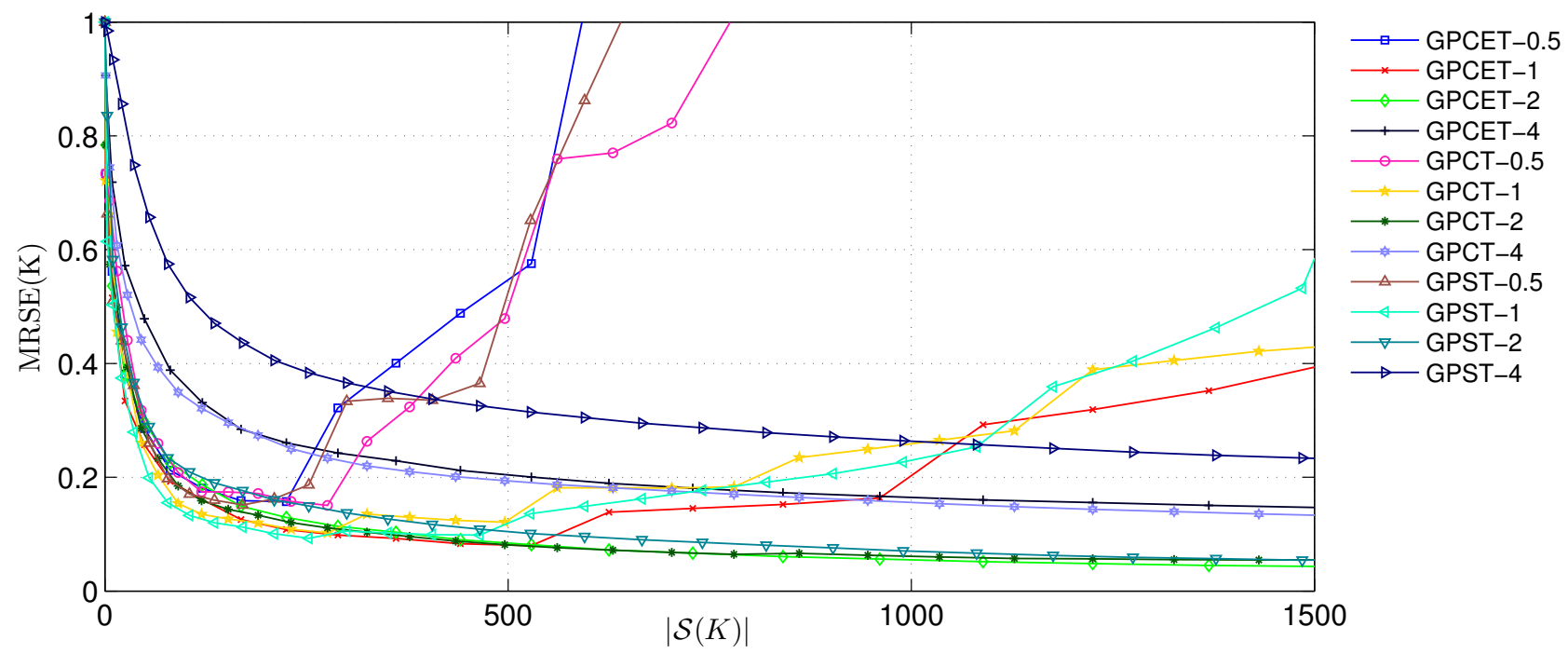

(c) $64 \times 64$

Figure 7: $\operatorname{MSRE}(K)$ curves of harmonic function-based methods (GPCET, GPCT, GPST) at $s=$ $0.5,1,2,4$ on the six character datasets (to be continued on the next page). 

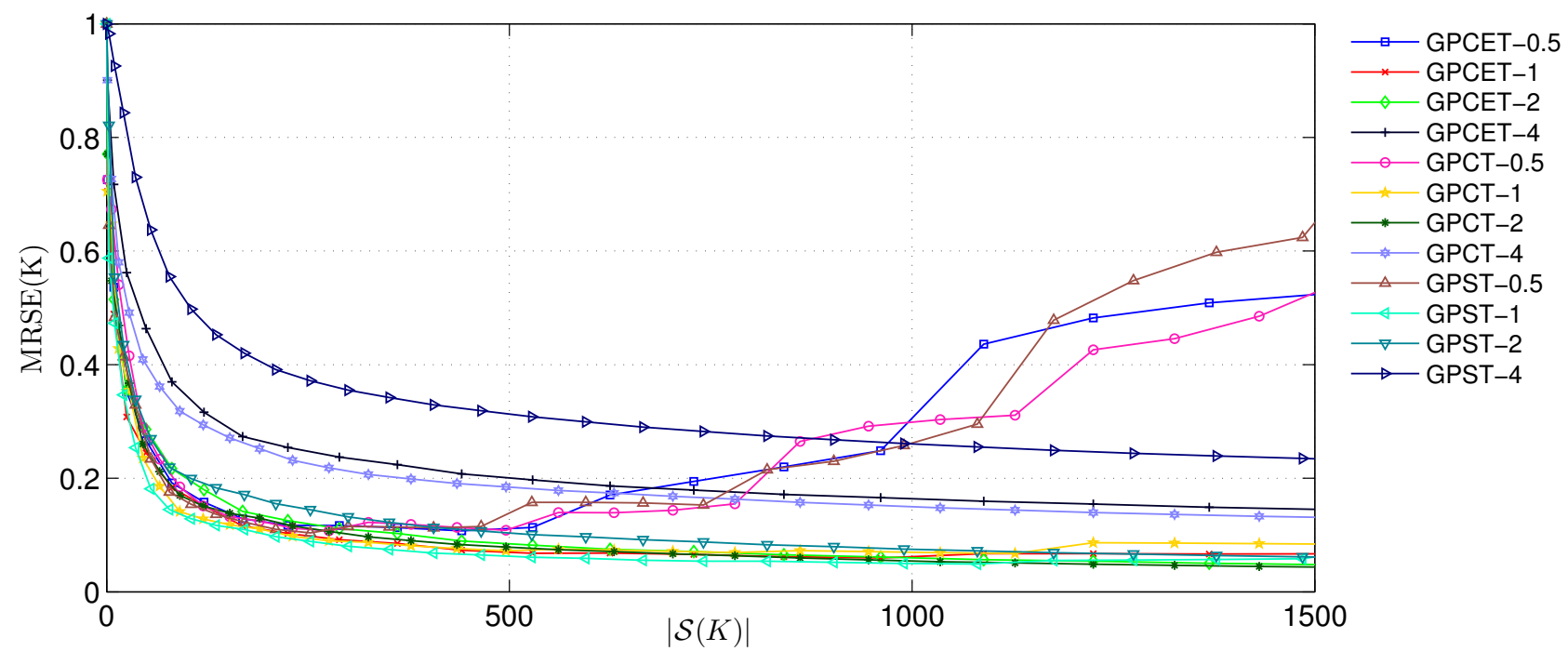

(d) $128 \times 128$
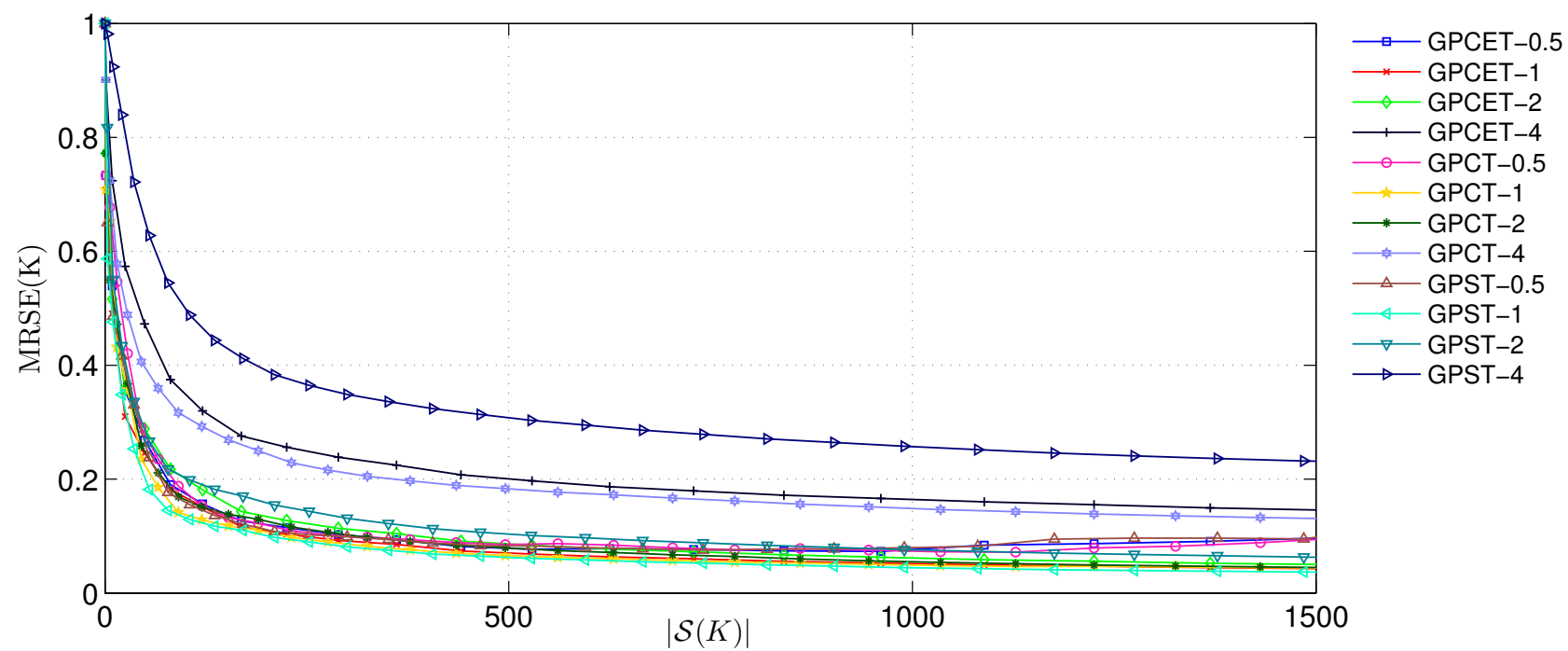

(e) $256 \times 256$
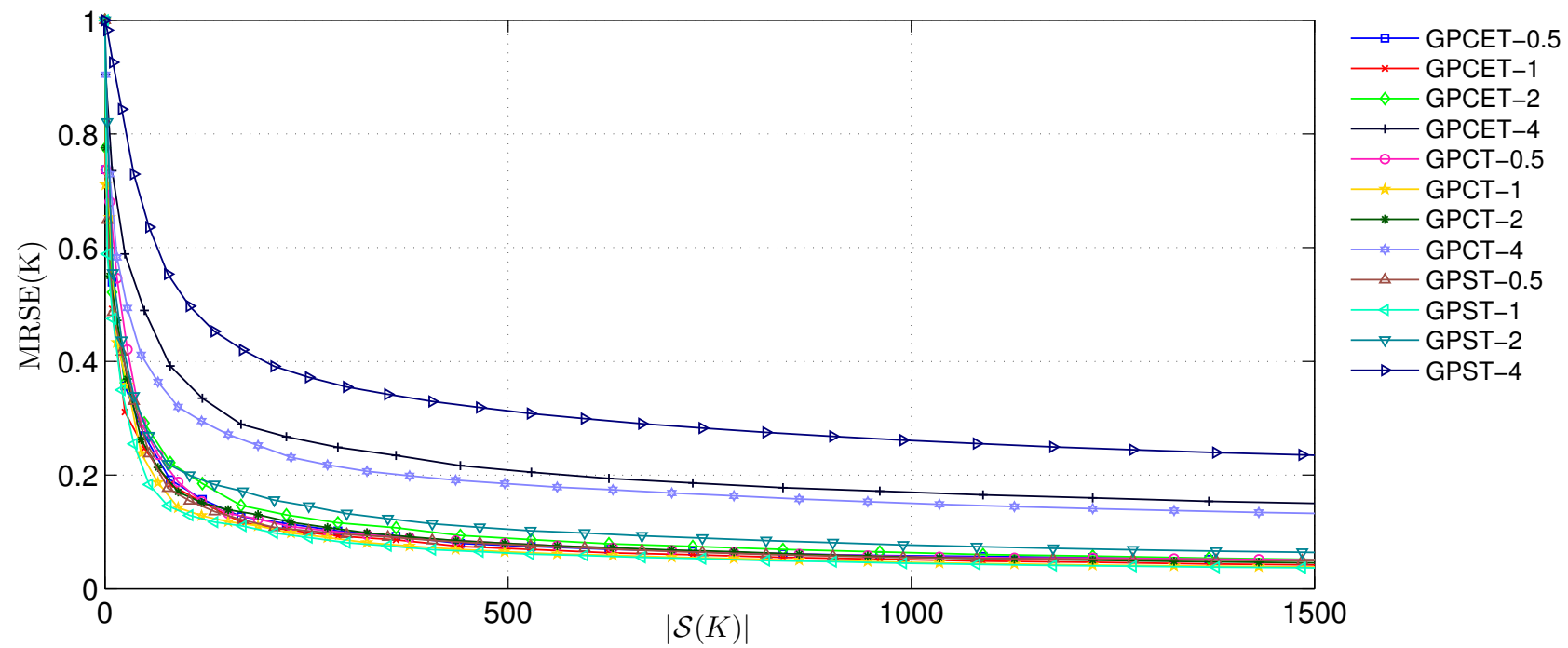

(f) $512 \times 512$

Figure 7: $\operatorname{MSRE}(K)$ curves of harmonic function-based methods (GPCET, GPCT, GPST) at $s=$ $0.5,1,2,4$ on the six character datasets. 


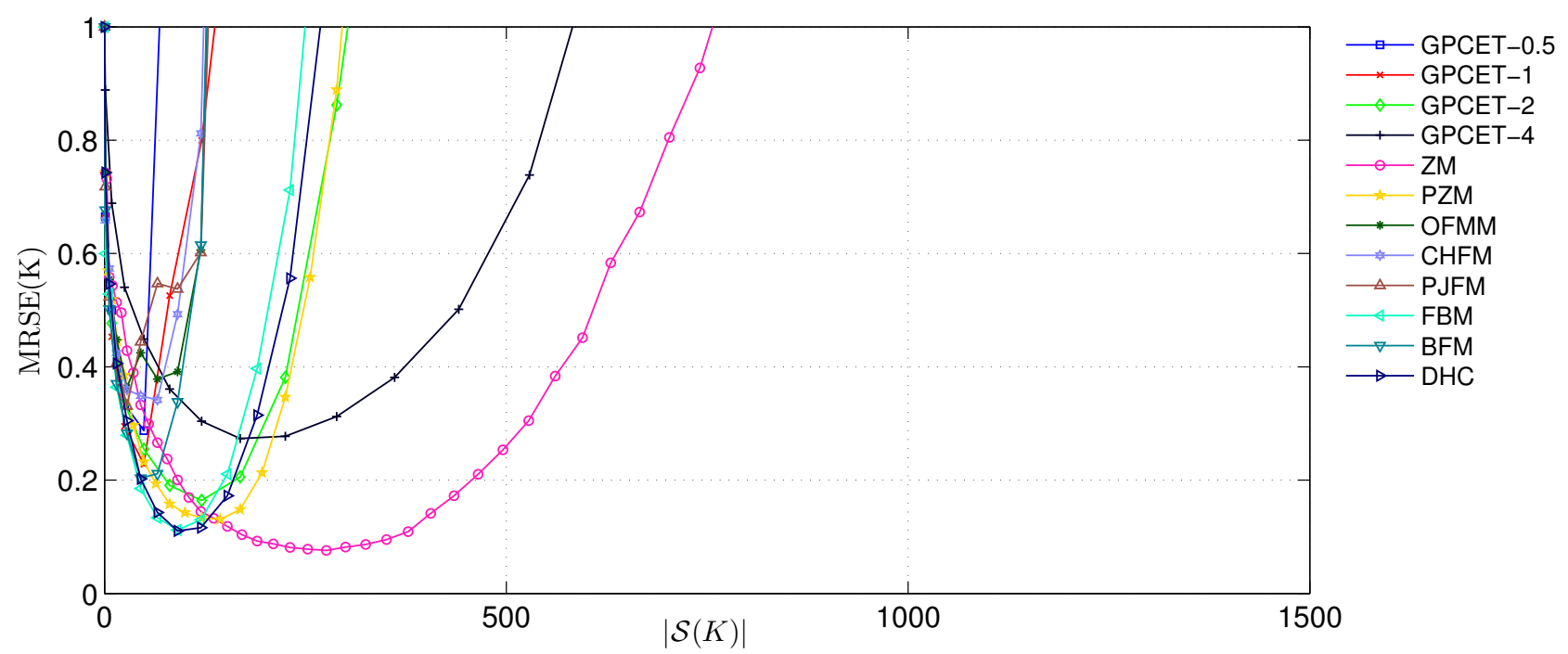

(a) $16 \times 16$
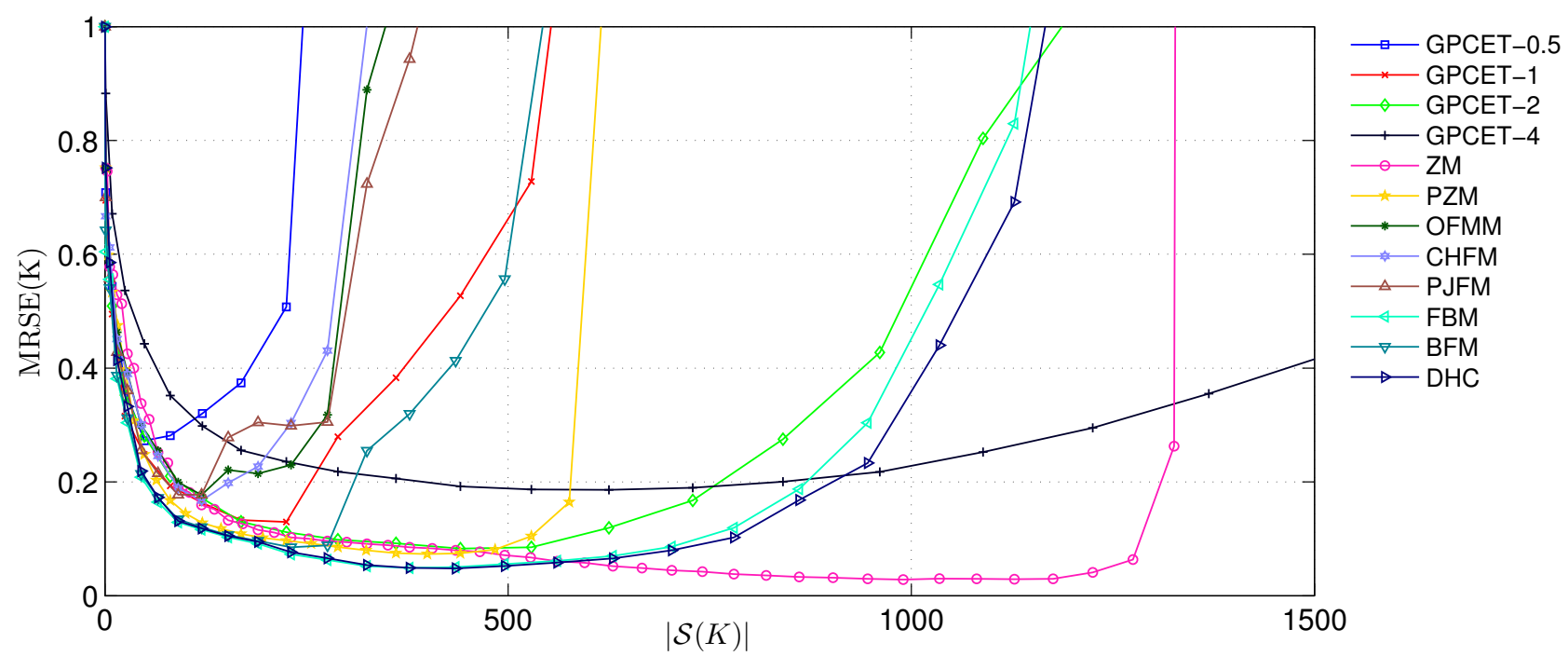

(b) $32 \times 32$
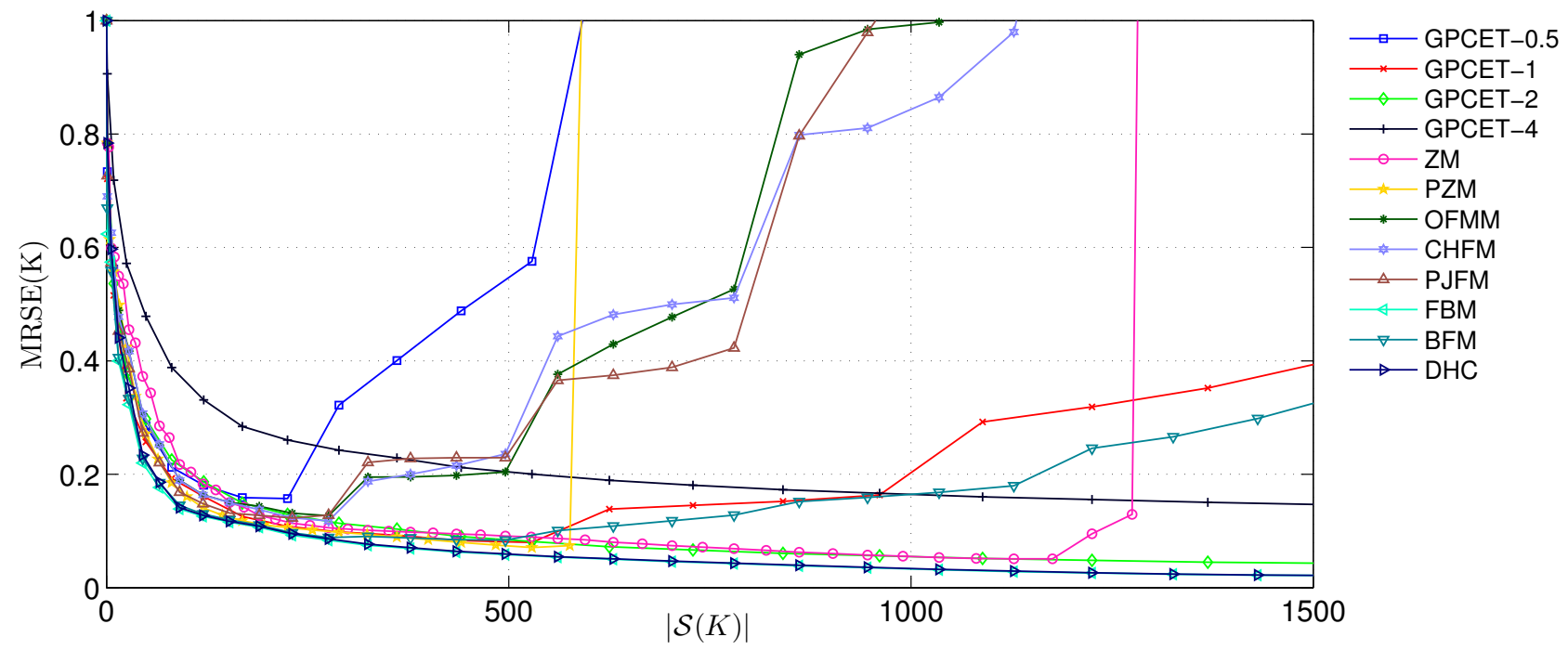

(c) $64 \times 64$

Figure 8: $\operatorname{MSRE}(K)$ curves of GPCET at $s=0.5,1,2,4$, Jacobi polynomial-based (ZM, PZM, OFMM, CHFM, PJFM), and eigenfunction-based (FBM, BFM, DHC) methods on the six character datasets (to be continued on the next page). 

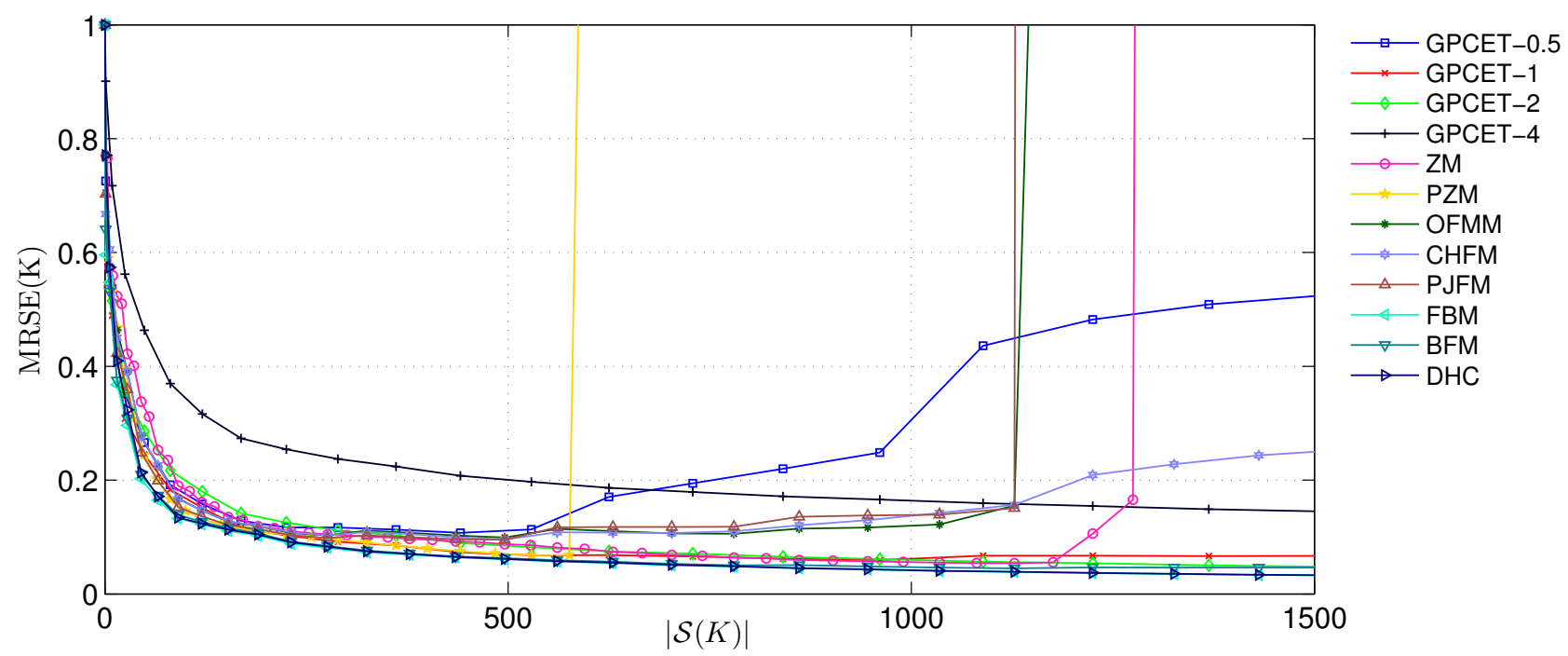

(d) $128 \times 128$

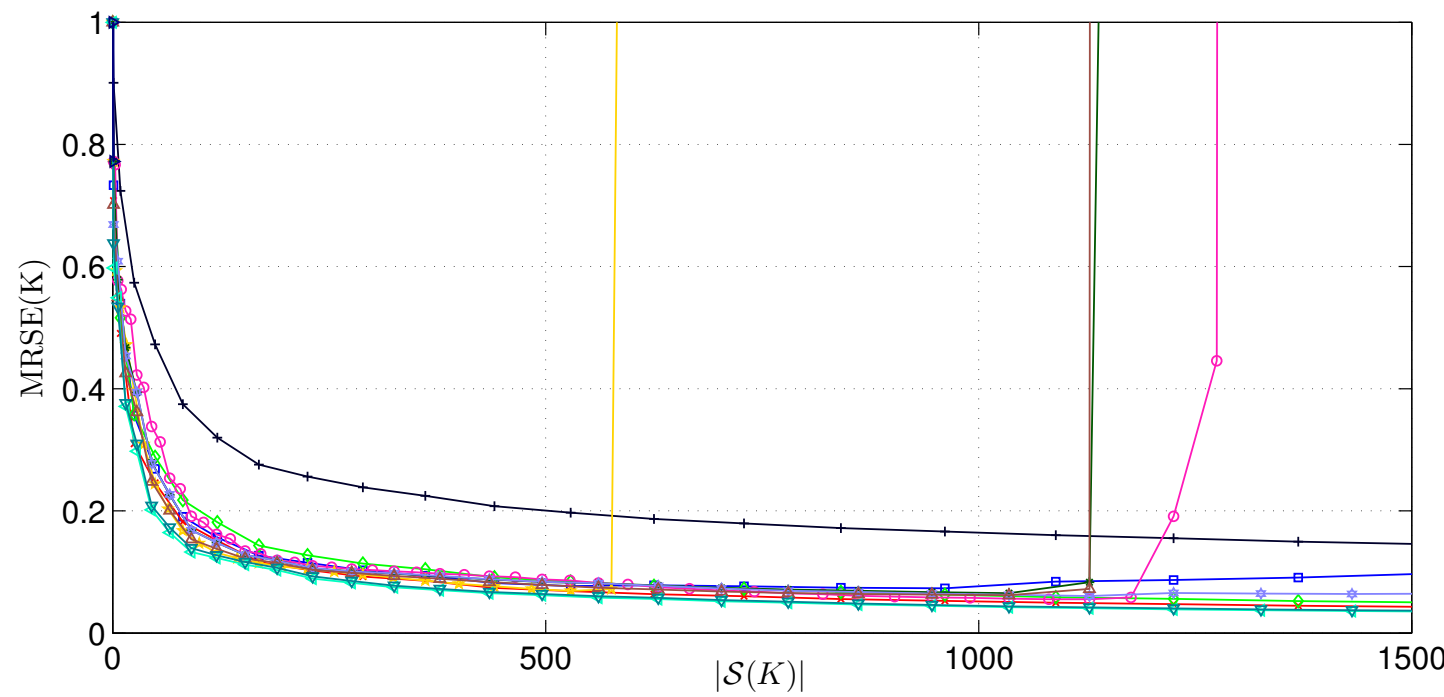

$\longrightarrow$ GPCET-0.5

* GPCET-1

$\checkmark$ GPCET-2

— GPCET-4

- ZM

* PZM

* OFMM

\#- CHFM

$\triangle$ PJFM

$\checkmark$ FBM

$\rightarrow$ BFM

$\longrightarrow$ DHC

(e) $256 \times 256$
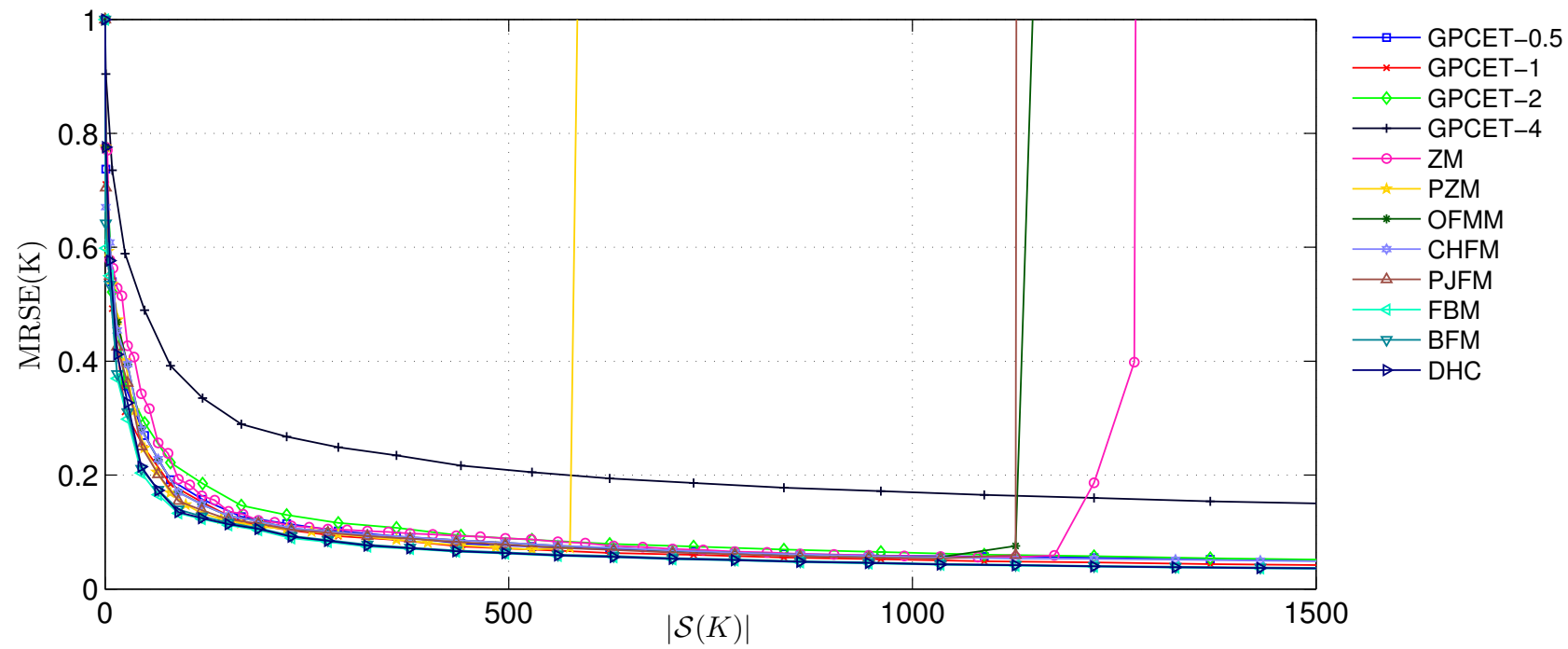

(f) $512 \times 512$

Figure 8: $\operatorname{MSRE}(K)$ curves of GPCET at $s=0.5,1,2,4$, Jacobi polynomial-based (ZM, PZM, OFMM, CHFM, PJFM), and eigenfunction-based (FBM, BFM, DHC) methods on the six character datasets. 
Table 1: Classification rates of harmonic function-based methods (GPCET, GRHFM, GPCT, GPST) at $s=0.5,1,2,4$ on NoiseAll dataset under different degrees of Gaussian noise $\sigma^{2}=0.00,0.05,0.10,0.15,0.20$ and at different values of $K=3,6,9,12,15$.

\begin{tabular}{|c|c|c|c|c|c|c|c|c|c|c|c|c|c|c|c|c|c|}
\hline \multirow{2}{*}{$K$} & \multirow{2}{*}{$\sigma^{2}$} & \multicolumn{4}{|c|}{ GPCET } & \multicolumn{4}{|c|}{ GRHFM } & \multicolumn{4}{|c|}{ GPCT } & \multicolumn{4}{|c|}{ GPST } \\
\hline & & $s=0.5$ & $s=1$ & $s=2$ & $s=4$ & $s=0.5$ & $s=1$ & $s=2$ & $s=4$ & $s=0.5$ & $s=1$ & $s=2$ & $s=4$ & $s=0.5$ & $s=1$ & $s=2$ & $s=4$ \\
\hline \multirow{5}{*}{3} & 0.00 & 0.00 & 100.00 & 100.00 & 100.00 & 0.00 & 100.00 & 100.00 & 100.00 & 00.00 & 100.00 & 100.00 & 100.00 & 100.00 & 100.00 & 100.00 & 100.00 \\
\hline & 0.05 & 98.00 & 98.00 & 97.08 & 96.08 & 8.00 & 97.92 & 97.75 & 97.00 & 96.92 & 97.67 & 96.75 & 94.67 & 95.75 & 96.58 & 95.67 & 87.92 \\
\hline & 0.10 & 87.67 & 81.67 & 75.25 & 78.00 & 86.58 & 87.08 & 81.58 & 81.83 & 75.67 & 83.83 & 75.08 & 75.92 & 72.58 & 78.75 & 70.50 & 70.17 \\
\hline & 0.15 & 57.00 & 56.00 & 47.75 & 49.42 & 59.50 & 65.42 & 56.50 & 50.42 & 46.33 & 53.75 & 46.67 & 47.00 & 45.33 & 50.75 & 42.00 & 41.33 \\
\hline & 20 & 34.75 & 35.08 & 27.67 & 28.25 & 38.42 & 41.25 & 33.25 & 32.08 & 28.33 & 33.08 & 27.42 & 28.33 & 28.17 & 32.00 & 22.08 & 3.00 \\
\hline \multirow{5}{*}{6} & 0.00 & 0.00 & 100.00 & 100.00 & 100.00 & 100.00 & 100.00 & 100.00 & 100.00 & 100.00 & 100.00 & 100.00 & 100.00 & 100.00 & 100.00 & 100.00 & 100.00 \\
\hline & 0.05 & 8.00 & 98.00 & 98.00 & 97.92 & 98.00 & 98.00 & 98.00 & 97.92 & 98.00 & 98.00 & 98.00 & 97.92 & 98.17 & 98.00 & 98.00 & 6.00 \\
\hline & 0.10 & 3.42 & 91.83 & 84.42 & 86.17 & 92.75 & 92.50 & 88.25 & 88.83 & 88.67 & 91.08 & 87.42 & 86.50 & 90.58 & 91.92 & .83 & 2.92 \\
\hline & 0.15 & 73.33 & 68.17 & 60.50 & 66.67 & 74.00 & 76.25 & 67.42 & 65.83 & 62.25 & 71.17 & 63.58 & 62.67 & 67.92 & 73.25 & 62.50 & 64.67 \\
\hline & 20 & 3.67 & 42.17 & 37.33 & 43.33 & .58 & 49.50 & 44.83 & & .42 & 42.92 & 39.92 & 3.50 & 42.83 & 46.92 & .00 & 40.58 \\
\hline \multirow{5}{*}{9} & 0.00 & 100.00 & 100.00 & 100.00 & 100.00 & 100.00 & 100.00 & 100.00 & 100.00 & 100.00 & 100.00 & 100.00 & 100.00 & 100.00 & 100.00 & 100.00 & 100.00 \\
\hline & 0.05 & 3.00 & 98.00 & 98.00 & 98.00 & 98.00 & 98.00 & 98.00 & 98.00 & 98.00 & 98.00 & 98.00 & 98.00 & 98.33 & 98.00 & 8.00 & 96.83 \\
\hline & 0.10 & 1.33 & 93.08 & 88.33 & 89.50 & 94.00 & 93.25 & 91.42 & 90.17 & .58 & 92.33 & 91.17 & 88.08 & 93.33 & 92.67 & .58 & 86.92 \\
\hline & 0.15 & 79.92 & 77.17 & 67.92 & 73.83 & 79.50 & 81.33 & 72.83 & 74.00 & 69.83 & 77.33 & 70.92 & 69.83 & 74.58 & 80.00 & 71.25 & 72.42 \\
\hline & 0.20 & 50.83 & 49.00 & 44.42 & 50.50 & 1.50 & 56.75 & 52.58 & 51.00 & 45.42 & 49.25 & 48.17 & 46.25 & 46.92 & 52.33 & 46.42 & 48.75 \\
\hline \multirow{5}{*}{12} & 0.00 & 100.00 & 100.00 & 100.00 & 100.00 & 100.00 & 100.00 & 100.00 & 100.00 & 100.00 & 100.00 & 100.00 & 100.00 & 100.00 & 100.00 & 100.00 & 100.00 \\
\hline & 0.05 & 98.08 & 98.00 & 98.00 & & 00 & 98.00 & 98.00 & .00 & .00 & 98.00 & 98.00 & 98.00 & 98.50 & 98.00 & 98.00 & 97.00 \\
\hline & 0.10 & 95.00 & 94.58 & 90.33 & 89.75 & 95.00 & 94.58 & 93.25 & 91.42 & 93.00 & 93.17 & 92.58 & 89.58 & 95.33 & 93.25 & 92.92 & 88.17 \\
\hline & 0.15 & 84.17 & 80.58 & 72.58 & 76.67 & 83.42 & 84.75 & 76.25 & 78.00 & 76.08 & 81.08 & 75.50 & 73.58 & 79.08 & 83.50 & 74.08 & 74.67 \\
\hline & 0.20 & 57.17 & 55.33 & 50.75 & 58.08 & 58.42 & 62.33 & 57.67 & 57.08 & 49.83 & 54.75 & 53.42 & 50.75 & 51.67 & 58.25 & 51.83 & 52.42 \\
\hline \multirow{5}{*}{15} & 0.00 & 100.00 & 100.00 & 100.00 & 100.00 & 100.00 & 100.00 & 100.00 & 100.00 & 100.00 & 100.00 & 100.00 & 100.00 & 100.00 & 100.00 & 100.00 & 100.00 \\
\hline & 0.05 & 98.17 & 98.00 & 98.00 & & 00 & 98.00 & 98.00 & 00 & .00 & 98.00 & .00 & .00 & 8.75 & 98.00 & 8.00 & 97.08 \\
\hline & 0.10 & 96.17 & 95.67 & 92.00 & 90.75 & 95.58 & 95.33 & 94.25 & 92.25 & 94.25 & 93.92 & 93.33 & 90.17 & 96.00 & 93.83 & 93.25 & 88.83 \\
\hline & 0.15 & 86.92 & 83.08 & 75.92 & 79.08 & 85.75 & 86.50 & 79.67 & 79.58 & 78.50 & 83.58 & 78.33 & 76.42 & 82.17 & 85.33 & 76.83 & 76.33 \\
\hline & 0.20 & 61.67 & 60.00 & 55.58 & 62.25 & 63.33 & 66.17 & 61.17 & 62.33 & 52.92 & 59.00 & 57.92 & 56.17 & 56.25 & 61.92 & 55.67 & 55.83 \\
\hline
\end{tabular}


Table 2: Classification rates of harmonic function-based methods (GPCET, GRHFM, GPCT, GPST) at $s=0.5,1,2,4$ on NoiseInner dataset under different degrees of Gaussian noise $\sigma^{2}=0.00,0.05,0.10,0.15,0.20$ and at different values of $K=1,2,3,4,5$.

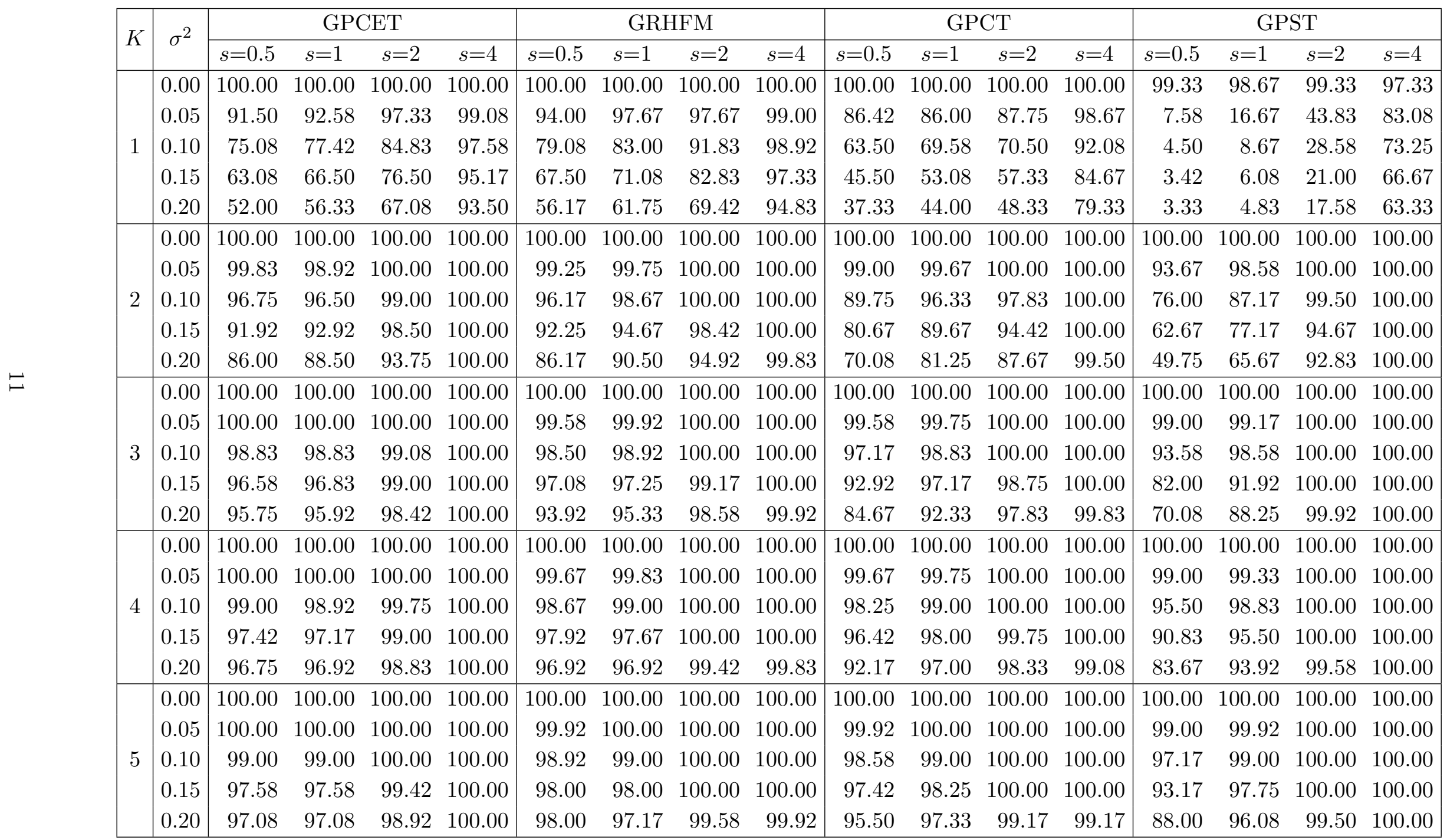


Table 3: Classification rates of harmonic function-based methods (GPCET, GRHFM, GPCT, GPST) at $s=0.5,1,2,4$ on NoiseOuter dataset under different degrees of Gaussian noise $\sigma^{2}=0.00,0.05,0.10,0.15,0.20$ and at different values of $K=2,4,6,8,10$.

\begin{tabular}{|c|c|c|c|c|c|c|c|c|c|c|c|c|c|c|c|c|c|}
\hline \multirow{2}{*}{$K$} & \multirow{2}{*}{$\sigma^{2}$} & \multicolumn{4}{|c|}{ GPCET } & \multicolumn{4}{|c|}{ GRHFM } & \multicolumn{4}{|c|}{ GPCT } & \multicolumn{4}{|c|}{ GPST } \\
\hline & & $s=0.5$ & $s=1$ & $s=2$ & $s=4$ & $=0.5$ & $s=1$ & $s=2$ & $s=4$ & $s=0.5$ & $s=1$ & $s=2$ & $s=4$ & $s=0.5$ & $s=1$ & $s=2$ & $s=4$ \\
\hline \multirow{5}{*}{2} & 0.00 & 0.00 & 100.00 & 100.00 & 100.00 & 0.00 & 100.00 & 100.00 & 100.00 & 00.00 & 100.00 & 100.00 & 100.00 & 00.00 & 100.00 & 100.00 & 100.00 \\
\hline & 0.05 & 98.00 & 97.17 & 97.08 & 94.25 & 7.42 & 98.08 & 96.08 & 94.08 & 4.33 & 95.08 & 92.92 & 93.25 & 5.67 & 92.58 & 80.17 & 79.58 \\
\hline & 0.10 & 95.42 & 86.25 & 75.58 & 75.83 & 92.92 & 91.00 & 82.58 & 79.08 & 76.83 & 85.83 & 72.17 & 70.08 & 79.25 & 69.75 & 53.17 & 47.42 \\
\hline & 0.15 & 79.67 & 73.00 & 57.42 & 46.17 & 82.75 & 78.33 & 65.25 & 52.92 & 57.67 & 64.08 & 55.83 & 45.42 & 64.33 & 46.67 & 32.17 & 25.08 \\
\hline & 0.20 & 65.75 & 59.58 & 42.83 & 29.50 & 7.92 & 65.25 & 52.25 & 34.92 & 46.58 & 51.00 & 40.08 & 31.08 & 49.00 & 33.92 & 20.92 & 16.33 \\
\hline \multirow{5}{*}{4} & 0.00 & 0.00 & 100.00 & 100.00 & 100.00 & 100.00 & 100.00 & 100.00 & 100.00 & 100.00 & 100.00 & 100.00 & 100.00 & 100.00 & 100.00 & 100.00 & 100.00 \\
\hline & 0.05 & .00 & 98.83 & 98.00 & 97.92 & 98.42 & 99.00 & 98.00 & 97.92 & 8.00 & 98.83 & 98.00 & 97.42 & 3.33 & 98.17 & 97.17 & 94.67 \\
\hline & 0.10 & 3.00 & 96.75 & 89.42 & 87.25 & 6.42 & 95.08 & 90.83 & 8.58 & 4.17 & 93.33 & 88.50 & 85.67 & .92 & 94.17 & .42 & 78.75 \\
\hline & 0.15 & 94.42 & 88.33 & 77.00 & 68.33 & 92.33 & 89.33 & 79.33 & 71.67 & 83.33 & 85.17 & 73.33 & 68.17 & 87.08 & 85.75 & 69.50 & 56.83 \\
\hline & 0.20 & .25 & 75.50 & 62.83 & 48.2 & 2.50 & 79.42 & 67.33 & .00 & 9.50 & 71.08 & 61.92 & 8.75 & 6.33 & 75.83 & .33 & 4.50 \\
\hline \multirow{5}{*}{6} & 0.00 & 100.00 & 100.00 & 100.00 & 100.00 & 100.00 & 100.00 & 100.00 & 100.00 & 100.00 & 100.00 & 100.00 & 100.00 & 100.00 & 100.00 & 100.00 & 100.00 \\
\hline & 0.05 & .00 & 99.00 & 98.00 & 98.00 & 98.92 & 99.00 & 98.00 & 98.00 & 98.00 & 99.00 & 98.00 & 98.00 & 99.00 & 99.00 & 98.00 & 96.67 \\
\hline & 0.10 & .00 & 98.00 & 93.25 & 89.33 & .00 & 97.58 & 94.67 & .92 & .75 & 95.08 & 93.00 & 8.83 & 7.00 & 96.17 & .58 & 84.42 \\
\hline & 0.15 & 6.17 & 91.17 & 83.75 & 77.17 & 93.08 & 92.00 & 85.08 & 79.42 & 88.92 & 90.67 & 82.25 & 77.50 & 92.25 & 90.83 & 79.00 & 68.50 \\
\hline & 0.20 & .50 & 82.67 & 70.75 & 58.58 & 3.17 & 84.58 & 73.42 & 1.25 & 77.75 & 80.00 & 69.42 & 57.42 & 86.83 & 84.08 & 62.33 & 45.67 \\
\hline \multirow{5}{*}{8} & 0.00 & 100.00 & 100.00 & 100.00 & 100.00 & 100.00 & 100.00 & 100.00 & 100.00 & 100.00 & 100.00 & 100.00 & 100.00 & 100.00 & 100.00 & 100.00 & 100.00 \\
\hline & 0.05 & 99.50 & 99.00 & 98.00 & 98.00 & 99.25 & 99.00 & 98.00 & 98.00 & 98.42 & 99.00 & 98.00 & 98.00 & 99.50 & 99.00 & 98.00 & 96.83 \\
\hline & 0.10 & 3.00 & 98.00 & 95.00 & 89.92 & 8.00 & 97.92 & 96.50 & 90.58 & 97.75 & 96.92 & 95.83 & 89.50 & 97.00 & 97.00 & 92.67 & 86.50 \\
\hline & 0.15 & 95.42 & 92.58 & 85.67 & 82.00 & 94.08 & 92.08 & 87.33 & 82.92 & 91.42 & 91.83 & 85.00 & 81.83 & 95.50 & 93.08 & 83.75 & 73.75 \\
\hline & 0.20 & .33 & 86.17 & 74.83 & 65.00 & 88.92 & 87.08 & 77.50 & 67.50 & 81.33 & 84.67 & 74.08 & 64.25 & 88.67 & 86.17 & 68.42 & 52.25 \\
\hline \multirow{5}{*}{10} & 0.00 & 100.00 & 100.00 & 100.00 & 100.00 & 100.00 & 100.00 & 100.00 & 100.00 & 100.00 & 100.00 & 100.00 & 100.00 & 100.00 & 100.00 & 100.00 & 100.00 \\
\hline & 0.05 & 99.58 & 99.00 & 98.00 & & & 99.00 & 98.00 & 00 & .58 & 99.00 & 98.00 & 98.00 & 100.00 & 99.00 & 98.00 & 97.00 \\
\hline & 0.10 & .00 & 98.00 & 96.50 & 90.17 & 98.00 & 97.92 & 97.17 & 91.00 & 98.00 & 97.42 & 96.50 & 90.00 & 97.00 & 97.50 & 93.50 & 88.00 \\
\hline & 0.15 & 95.58 & 93.08 & 87.25 & 83.58 & 94.50 & 92.17 & 88.50 & 84.17 & 92.25 & 91.92 & 86.33 & 83.75 & 95.92 & 93.17 & 85.17 & 76.00 \\
\hline & 0.20 & 91.75 & 87.67 & 78.17 & 70.42 & 89.67 & 88.67 & 79.92 & 72.33 & 83.58 & 86.92 & 76.42 & 69.33 & 91.08 & 88.00 & 73.50 & 58.00 \\
\hline
\end{tabular}


Table 4: Classification rates of GRHFM at $s=0.5,1,2,4$, non-orthogonal (ART, GFD, RM), Jacobi polynomial-based (ZM, PZM, OFMM, CHFM, PJFM), and eigenfunction-based (FBM, BFM, DHC) methods on NoiseAll dataset under different degrees of Gaussian noise $\sigma^{2}=$ $0.00,0.05,0.10,0.15,0.20$ and at different values of $K=3,6,9,12,15$.

\begin{tabular}{|c|c|c|c|c|c|c|c|c|c|c|c|c|c|c|c|c|}
\hline \multirow{2}{*}{$K$} & \multirow{2}{*}{$\sigma^{2}$} & \multicolumn{4}{|c|}{ GRHFM } & \multirow{2}{*}{ ART } & \multirow{2}{*}{ GFD } & \multirow{2}{*}{ RM } & \multirow{2}{*}{ ZM } & \multirow{2}{*}{ PZM } & \multirow{2}{*}{ OFMM } & \multirow{2}{*}{ CHFM } & \multirow{2}{*}{ PJFM } & \multirow{2}{*}{ FBM } & \multirow{2}{*}{ BFM } & \multirow{2}{*}{ DHC } \\
\hline & & $s=0.5$ & $s=1$ & $s=2$ & $=4$ & & & & & & & & & & & \\
\hline \multirow{5}{*}{3} & 0.00 & 100.00 & 100.00 & 100.00 & 100.00 & 100.00 & 100.00 & 100.00 & 100.00 & 100.00 & 100.00 & 100.00 & 100.00 & 100.00 & 100.00 & 100.00 \\
\hline & 0.05 & 98.00 & 97.92 & 97.75 & 97.00 & 96.75 & 97.92 & 79.25 & 78.67 & 93.83 & 97.42 & 97.92 & 96.50 & 97.33 & 97.92 & 96.42 \\
\hline & 0.10 & 86.58 & 87.08 & 81.58 & 81.83 & 79.92 & 83.50 & 39.17 & 43.92 & 68.00 & 77.00 & 78.50 & 79.58 & 87.67 & 84.42 & 83.50 \\
\hline & 0.15 & 59.50 & 65.42 & 56.50 & 50.42 & 51.50 & 54.50 & 19.33 & 20.42 & 43.00 & 55.50 & 53.67 & 55.92 & 66.58 & 65.67 & 55.25 \\
\hline & 0.20 & 38.42 & 41.25 & 33.25 & 32.08 & 28.67 & 31.92 & 11.17 & 9.67 & 26.08 & 36.83 & 31.67 & 32.50 & 42.33 & 39.67 & 34.83 \\
\hline \multirow{5}{*}{6} & 0.00 & 100.00 & 100.00 & 100.00 & 100.00 & 100.00 & 100.00 & 100.00 & 100.00 & 100.00 & 100.00 & 100.00 & 100.00 & 100.00 & 100.00 & 100.00 \\
\hline & 0.05 & 98.00 & 98.00 & 98.00 & 97.92 & 98.00 & 98.00 & 93.08 & 97.58 & 98.00 & 98.00 & 98.00 & 98.00 & 98.25 & 98.00 & 98.00 \\
\hline & 0.10 & 92.75 & 92.50 & 88.25 & 88.83 & 89.75 & 91.83 & 63.50 & 84.58 & 90.58 & 92.83 & 89.75 & 89.08 & 94.50 & 94.42 & 93.33 \\
\hline & 0.15 & 74.00 & 76.25 & 67.42 & 65.83 & 68.58 & 67.42 & 36.00 & 56.75 & 67.75 & & & 67.00 & 81.67 & 82.83 & 75.67 \\
\hline & 0.20 & 45.58 & 49.50 & 44.83 & 43.83 & 44.75 & 41.67 & 20.67 & 35.00 & 44.33 & 54.00 & 42.00 & 44.58 & 57.42 & 56.83 & 52.92 \\
\hline \multirow{5}{*}{9} & & 100.00 & 100.00 & 100.00 & 100.00 & 100.00 & 100.00 & & 100.00 & & & & 0.00 & 100.00 & & 100.00 \\
\hline & 0.05 & 8.00 & 98.00 & 98.00 & 98.00 & 98.00 & 98.00 & .50 & 97.92 & .00 & & .00 & .00 & 99.00 & & 98.00 \\
\hline & 0.10 & 94.00 & 93.25 & 91.42 & 90.17 & 92.17 & 93.67 & & 90.50 & 93.08 & & & 91.17 & 95.67 & 95.50 & 94.25 \\
\hline & 0.15 & 9.50 & 81.33 & 72.83 & 74.00 & 75.83 & 74.92 & 45.00 & 72.08 & & & & 74.42 & 85.92 & 86.33 & 83.42 \\
\hline & 0.20 & 51.50 & 56.75 & 52.58 & 51.00 & 50.17 & 49.17 & 26.17 & 44.83 & 55.58 & 64.33 & 48.08 & 49.08 & 68.33 & 67.17 & 64.42 \\
\hline \multirow{5}{*}{12} & 0.00 & 100.00 & 100.00 & 100.00 & 100.00 & 100.00 & 100.00 & 100.00 & 100.00 & 100.00 & 100 & 100.00 & 100.00 & 100.00 & 100.00 & 100.00 \\
\hline & 0.05 & 98.00 & 98.00 & 98.00 & 98.00 & 98.00 & 98.00 & 97.25 & 98.00 & 98.00 & .08 & 98.00 & 98.00 & 99.00 & 98.00 & 98.00 \\
\hline & 0.10 & 95.00 & 94.58 & 93.25 & 91.42 & 93.33 & 94.17 & 77.08 & 93.33 & 93.92 & 96.00 & 92.67 & 92.25 & 96.50 & 96.08 & 95.50 \\
\hline & 0.15 & 83.42 & 84.75 & 76.25 & 78.00 & & & & & & & & 77.83 & 90.17 & 91.25 & 88.67 \\
\hline & 0.20 & 58.42 & 62.33 & 57.67 & 57.08 & 54.33 & 53.83 & 28.92 & 56.08 & 63.42 & 71.75 & 54.58 & 54.17 & 74.42 & 76.08 & 71.42 \\
\hline \multirow{5}{*}{15} & 0.00 & 100.00 & 100.00 & 100.00 & 100.00 & 100.00 & 100.00 & 100.00 & 100.00 & 100.00 & 100.00 & 100.00 & 100.00 & 100.00 & 100.00 & 100.00 \\
\hline & 0.05 & 98.00 & 98.00 & 98.00 & 98.00 & & & & & & & & & & 98.25 & 98.25 \\
\hline & 0.10 & 95.58 & 95.33 & 94.25 & 92.25 & 94.08 & 94.42 & 79.67 & 94.50 & 94.42 & 96.42 & 93.50 & 92.92 & 97.58 & 96.50 & 95.92 \\
\hline & 0.15 & 85.75 & 86.50 & 79.67 & 79.58 & 80.33 & 81.42 & 51.75 & 81.08 & 85.08 & 89.67 & 80.00 & 80.75 & 92.75 & 93.00 & 91.33 \\
\hline & 0.20 & 63.33 & 66.17 & 61.17 & 62.33 & 58.75 & 57.83 & 30.83 & 60.83 & 67.33 & 75.67 & 58.17 & 57.17 & 79.75 & 81.50 & 76.17 \\
\hline
\end{tabular}


Table 5: Classification rates of GRHFM at $s=0.5,1,2,4$, non-orthogonal (ART, GFD, RM), Jacobi polynomial-based (ZM, PZM, OFMM, CHFM, PJFM), and eigenfunction-based (FBM, BFM, DHC) methods on NoiseInner dataset under different degrees of Gaussian noise $\sigma^{2}=$ $0.00,0.05,0.10,0.15,0.20$ and at different values of $K=1,2,3,4,5$.

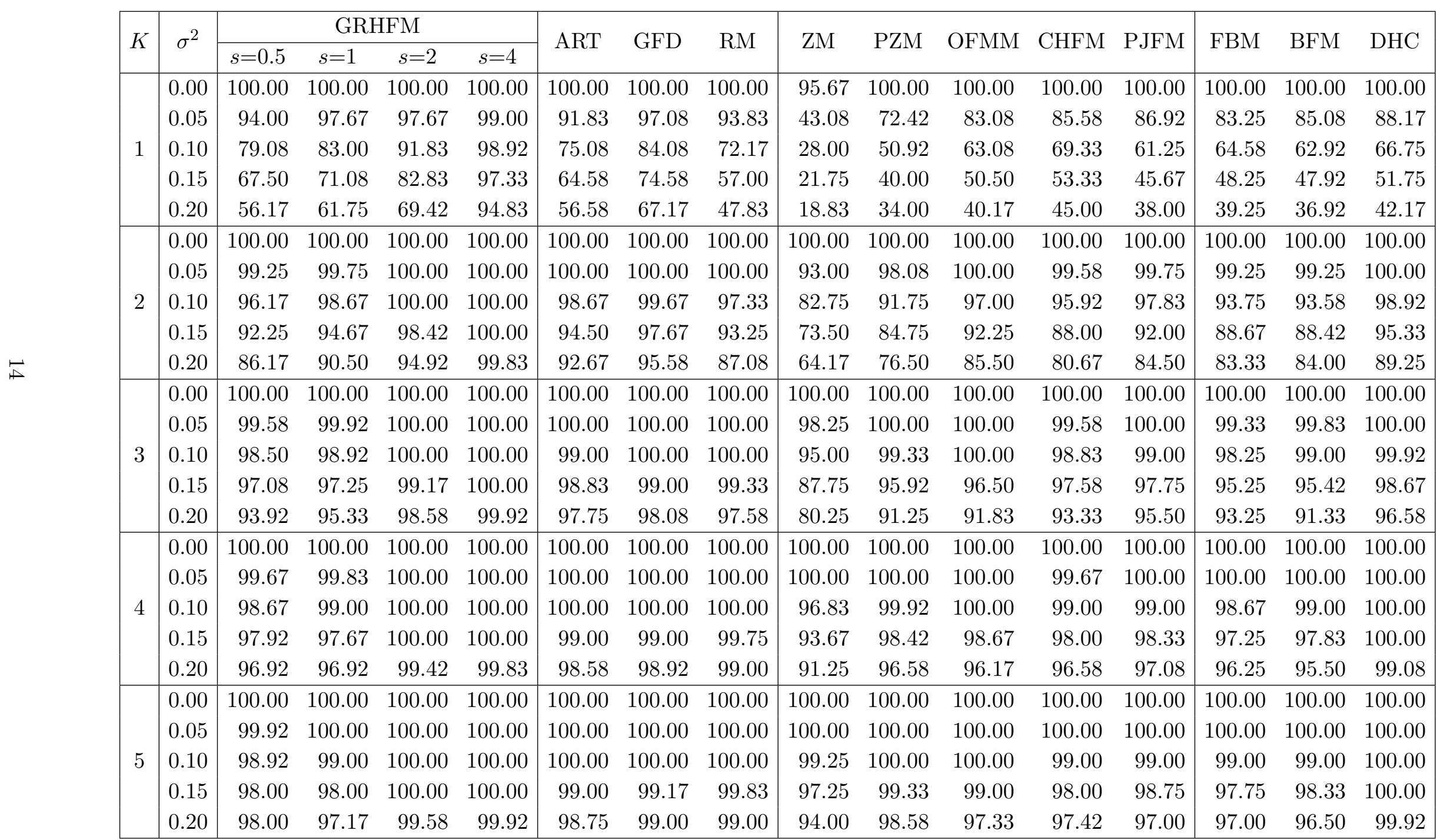


Table 6: Classification rates of GRHFM at $s=0.5,1,2,4$, non-orthogonal (ART, GFD, RM), Jacobi polynomial-based (ZM, PZM, OFMM, CHFM, PJFM), and eigenfunction-based (FBM, BFM, DHC) methods on NoiseOuter dataset under different degrees of Gaussian noise $\sigma^{2}=$ $0.00,0.05,0.10,0.15,0.20$ and at different values of $K=2,4,6,8,10$.

\begin{tabular}{|c|c|c|c|c|c|c|c|c|c|c|c|c|c|c|c|c|}
\hline \multirow{2}{*}{$K$} & \multirow{2}{*}{$\sigma^{2}$} & \multicolumn{4}{|c|}{ GRHFM } & \multirow{2}{*}{ ART } & \multirow{2}{*}{ GFD } & \multirow{2}{*}{ RM } & \multirow{2}{*}{$\mathrm{ZM}$} & \multirow{2}{*}{ PZM } & \multirow{2}{*}{ OFMM } & \multirow{2}{*}{ CHFM } & \multirow{2}{*}{ PJFM } & \multirow{2}{*}{ FBM } & \multirow{2}{*}{ BFM } & \multirow{2}{*}{ DHC } \\
\hline & & $s=0.5$ & $s=1$ & $s=2$ & $s=4$ & & & & & & & & & & & \\
\hline \multirow{5}{*}{2} & 0.00 & 100.00 & 100.00 & 100.00 & 100.00 & 100.00 & 100.00 & 100.00 & 100.00 & 100.00 & 100.00 & 100.00 & 100.00 & 100.00 & 100.00 & 100.00 \\
\hline & 0.05 & 97.42 & 98.08 & 96.08 & 94.08 & 91.42 & 96.08 & 70.75 & 59.50 & 87.17 & 93.00 & 93.83 & 93.67 & 98.08 & 95.25 & 96.33 \\
\hline & 0.10 & 92.92 & 91.00 & 82.58 & 79.08 & 74.00 & 81.33 & 37.00 & 21.42 & 62.17 & 76.42 & 79.17 & 81.42 & 89.33 & 85.17 & 84.00 \\
\hline & 0.15 & 82.75 & 78.33 & 65.25 & 52.92 & 52.75 & 63.08 & 21.58 & 9.83 & 41.58 & 64.33 & 64.17 & 63.58 & 76.08 & 73.00 & 61.75 \\
\hline & 0.20 & 67.92 & 65.25 & 52.25 & 34.92 & 32.50 & 46.00 & 15.08 & 7.42 & 28.67 & 50.83 & 51.67 & 51.33 & 58.25 & 57.08 & 46.92 \\
\hline \multirow{5}{*}{4} & 0.00 & 100.00 & 100.00 & 100.00 & 100.00 & 100.00 & 100.00 & 100.00 & 00.00 & 100.00 & 100.00 & 100.00 & 100.00 & 100.00 & 100.00 & 100.00 \\
\hline & 0.05 & 98.42 & 99.00 & 98.00 & 97.92 & 97.92 & 98.00 & 91.83 & 92.58 & 98.00 & 98.00 & 98.25 & 98.00 & 99.67 & 98.17 & 98.00 \\
\hline & 0.10 & 96.42 & 95.08 & 90.83 & 88.58 & 87.58 & 91.33 & 66.00 & 77.08 & 91.67 & 92.92 & 92.83 & 92.92 & 96.92 & 95.83 & 95.17 \\
\hline & 0.15 & 92.33 & 89.33 & 79.33 & 71.67 & 73.17 & 77.58 & 37.75 & 57.17 & 74.08 & .50 & 84. & 83.17 & 94.00 & 88. & 86.25 \\
\hline & 0.20 & 82.50 & 79.42 & 67.33 & 52.00 & 56.75 & 58.42 & 20.50 & 38.17 & 60.75 & 73.75 & 70.08 & 68.58 & 86.58 & 79.92 & 76.25 \\
\hline \multirow{5}{*}{6} & 0.00 & 0.00 & 100.00 & 100.00 & 100.00 & 100.00 & 100.00 & 100.00 & 100.00 & 100.00 & .00 & 100. & 00.00 & 100.00 & 100.00 & 100.00 \\
\hline & 0.05 & 98.92 & 99.00 & 98.00 & 98.00 & 98.00 & .00 & 95.08 & 98.00 & 08 & & & 98.00 & 100.00 & 9.08 & 99.67 \\
\hline & 0.10 & 98.00 & 97.58 & 94.67 & 89.92 & 92.58 & 94.08 & 74.08 & 89.42 & 94.83 & 96.92 & 94.00 & 94.25 & 98.67 & 97.92 & 97.00 \\
\hline & 0.15 & 93.08 & 92.00 & 85.08 & 79.42 & 81.67 & 84.42 & 47.42 & 75.83 & 87.33 & 88.00 & 88.00 & 88.00 & 95.50 & 93.75 & 91.33 \\
\hline & 0.20 & 88.17 & 84.58 & 73.42 & 61.25 & 63.33 & 65.00 & 30.58 & 57.33 & 76.00 & 79.25 & 76.92 & 77.17 & 91.92 & 88.08 & 84.50 \\
\hline \multirow{5}{*}{8} & 0.00 & 100.00 & 100.00 & 100.00 & 100.00 & 100.00 & 100.00 & 100.00 & 100.00 & 100.00 & 100.00 & 100.00 & 100.00 & 100.00 & 100.00 & 100.00 \\
\hline & 0.05 & 99.25 & 99.00 & 98.00 & 98.00 & 98.00 & 98.00 & 96.17 & 98.00 & 99.17 & 99.00 & 99.00 & 98.00 & 100.00 & 100.00 & 100.00 \\
\hline & 0.10 & 98.00 & 97.92 & 96.50 & 90.58 & 94.25 & 95.75 & 81.42 & 93.25 & 96.50 & 97.33 & 96.17 & 95.58 & 98.92 & 98.00 & 97.75 \\
\hline & 0.15 & 94.08 & 92.08 & 87.33 & 82.92 & 85.25 & 86.00 & 52.42 & 84.42 & 89.42 & 89.50 & 90.08 & 89.75 & 96.67 & 94.67 & 93.50 \\
\hline & 0.20 & 88.92 & 87.08 & 77.50 & 67.50 & 69.50 & 68.83 & 35.25 & 71.33 & 82.17 & 83.92 & 80.67 & 81.33 & 93.17 & 90.67 & 87.42 \\
\hline \multirow{5}{*}{10} & 0.00 & 100.00 & 100.00 & 100.00 & 100.00 & 100.00 & 100.00 & 100.00 & 100.00 & 100.00 & 100.00 & 100.00 & 100.00 & 100.00 & 100.00 & 100.00 \\
\hline & 0.05 & 99.42 & 99.00 & 98.00 & 98.00 & 98.00 & 98.00 & 97.50 & 98.33 & 99.83 & 99.42 & 99.00 & 98.00 & 100.00 & 100.00 & 100.00 \\
\hline & 0.10 & 98.00 & 97.92 & 97.17 & 91.00 & 95.17 & 96.67 & 84.33 & 96.17 & 97.42 & 97.83 & 97.58 & 97.42 & 99.00 & 98.00 & 98.08 \\
\hline & 0.15 & 94.50 & 92.17 & 88.50 & 84.17 & 85.92 & & & 87.50 & 91.33 & 92.17 & 91.25 & 90.67 & 97.17 & 95.17 & 94.50 \\
\hline & 0.20 & 89.67 & 88.67 & 79.92 & 72.33 & 73.67 & 72.17 & 38.83 & 77.08 & 84.17 & 86.42 & 84.75 & 82.50 & 94.75 & 92.75 & 88.58 \\
\hline
\end{tabular}

